\title{
Search for resonances and quantum black holes using dijet mass spectra in proton-proton collisions at $\sqrt{s}=8 \mathrm{TeV}$
}

\author{
V. Khachatryan et al. \\ (CMS Collaboration) \\ (Received 17 January 2015; published 12 March 2015)
}

\begin{abstract}
A search for resonances and quantum black holes is performed using the dijet mass spectra measured in proton-proton collisions at $\sqrt{s}=8 \mathrm{TeV}$ with the CMS detector at the LHC. The data set corresponds to an integrated luminosity of $19.7 \mathrm{fb}^{-1}$. In a search for narrow resonances that couple to quark-quark, quarkgluon, or gluon-gluon pairs, model-independent upper limits, at $95 \%$ confidence level, are obtained on the production cross section of resonances, with masses above $1.2 \mathrm{TeV}$. When interpreted in the context of specific models the limits exclude string resonances with masses below $5.0 \mathrm{TeV}$; excited quarks below 3.5 TeV; scalar diquarks below 4.7 TeV; $W^{\prime}$ bosons below $1.9 \mathrm{TeV}$ or between 2.0 and $2.2 \mathrm{TeV}$; $Z^{\prime}$ bosons below $1.7 \mathrm{TeV}$; and Randall-Sundrum gravitons below 1.6 TeV. A separate search is conducted for narrow resonances that decay to final states including $b$ quarks. The first exclusion limit is set for excited $b$ quarks, with a lower mass limit between 1.2 and $1.6 \mathrm{TeV}$ depending on their decay properties. Searches are also carried out for wide resonances, assuming for the first time width-to-mass ratios up to $30 \%$, and for quantum black holes with a range of model parameters. The wide resonance search excludes axigluons and colorons with mass below $3.6 \mathrm{TeV}$, and color-octet scalars with mass below $2.5 \mathrm{TeV}$. Lower bounds between 5.0 and $6.3 \mathrm{TeV}$ are set on the masses of quantum black holes.
\end{abstract}

DOI: 10.1103/PhysRevD.91.052009

PACS numbers: 13.85.Rm, 13.87.Ce, 14.80.-j

\section{INTRODUCTION}

We report on a search for new states decaying to dijets in proton-proton $(p p)$ collisions at a center-of-mass energy of $\sqrt{s}=8 \mathrm{TeV}$. The data sample corresponds to an integrated luminosity of $19.7 \mathrm{fb}^{-1}$ collected with the CMS detector at the CERN LHC in 2012. This analysis extends the search for new phenomena presented in previous CMS [1-6] and ATLAS [7-11] publications. A review of experimental searches for new particles in the dijet mass spectrum is presented in Ref. [12].

Many extensions of the standard model (SM) predict the existence of new massive particles that couple to quarks or antiquarks $(q)$ and gluons $(g)$. These new particles could produce resonant bumps in the dijet invariant mass distribution associated with strong interaction processes. A similar signature could be produced by quantum black holes $(\mathrm{QBH})$ that decay primarily to dijet final states.

Four studies are reported in this paper: (i) a search for narrow dijet resonances using the inclusive mass spectrum, with different sensitivities to the masses of $q q, q g$, and $g g$ final states; (ii) a dedicated search for narrow resonances decaying to $b$ quarks; (iii) a search for wide dijet resonances in the $q q$ and $g g$ final states; and (iv) a search for QBHs decaying to two jets.

* Full author list given at the end of the article.

Published by the American Physical Society under the terms of the Creative Commons Attribution 3.0 License. Further distribution of this work must maintain attribution to the author(s) and the published articles title, journal citation, and DOI.
We interpret the results in the context of particles predicted by several representative models: string resonances (S) $[13,14]$; scalar diquarks (D) [15]; excited quarks $\left(q^{*}\right)$ $[16,17]$ including excited $b$ quarks $\left(b^{*}\right)$; axigluons (A) [18-20]; color-octet colorons (C) [21]; the color-octet scalar (S8) resonances [22]; new gauge bosons $\left(W^{\prime}\right.$ and $\left.Z^{\prime}\right)$ [23] with SM-like couplings (SSM); Randall-Sundrum (RS) gravitons (g) [24-26]; and QBHs [27-29]. More details on the specific choices of couplings and the cross sections assumed for the models considered can be found in Ref. [5].

Narrow resonances are considered to be those that have small natural widths compared to the experimental dijet mass resolution. We search for narrow $q q$ and $g g$ resonances using the predicted dijet resonance line shape of the RS graviton model for the parameter choice $k / \bar{M}_{\mathrm{Pl}}=0.1$, where $k$ is the unknown curvature scale of the extra dimension and $\bar{M}_{\mathrm{Pl}}$ is the reduced Planck scale. This choice corresponds to a natural width equal to $1.5 \%$ of the resonance mass.

To search for narrow resonances decaying to $b$ quarks, the events are divided into samples with zero, one, or two jets identified as originating from $b$ quarks. These samples are labeled $0 b, 1 b$, and $2 b$, respectively. The sensitivity of the search to a given signal model depends on whether the predicted $0 b$ and $1 b$ resonant samples are dominated by gluons or quarks in the final state. Therefore two scenarios are considered: resonances that decay predominantly into pairs of gluons or $b$ quarks (" $g g / b b$ ") or resonances that decay predominantly into quark pairs only (" $q q / b b$ "). Dijet mass shapes appropriate to $g g$ resonances or $q q$ resonances are used in conjunction with $b b$ mass shapes. The dijet mass shapes in each tag category are weighted according to the expected gluon, quark, or $b$-quark content. 
Wide resonances are considered to be those where the natural width is comparable to or larger than the experimental dijet mass resolution. The signature for a wide resonance would be a broad enhancement in the dijet mass distribution. Wide $q q$ and $g g$ resonances are considered using the dijet resonance line shape of the RS graviton model with larger values of $k / \bar{M}_{\mathrm{Pl}}$, which correspond to natural widths up to $30 \%$ of the resonance mass.

Using the same technique employed in the inclusive analysis, we search for QBHs decaying to dijet final states. The search is motivated by theories in which the effective Planck scale in the presence of extra dimensions $\left(M_{\mathrm{D}}\right)$ is significantly smaller than the nominal Planck scale $\left(M_{\mathrm{Pl}} \sim 10^{16} \mathrm{TeV}\right)$, as for instance in the Arkani-HamedDimopoulos-Dvali (ADD) model [30,31] of flat extra dimensions or the RS model [24-26] of warped extra dimensions. The dijet mass spectrum for QBHs is characterized by a peaking structure, as a result of the opening of the $\mathrm{QBH}$ production threshold for parton center-of-mass energies above the minimum mass $M_{\mathrm{QBH}}^{\min }$ of $\mathrm{QBHs}$ and the steeply falling parton luminosity at higher center-of-mass energies. This shape differs from a resonance line shape and is almost independent of both the number of extra dimensions $n$ and the scale $M_{\mathrm{D}}$.

\section{THE CMS DETECTOR}

The central feature of the CMS apparatus [32] is a superconducting solenoid of $6 \mathrm{~m}$ internal diameter providing an axial field of $3.8 \mathrm{~T}$. Within the field volume are located the silicon pixel and strip tracker and the barrel and endcap calorimeters; a lead tungstate crystal electromagnetic calorimeter and a brass and scintillator hadron calorimeter. An iron/quartz-fiber calorimeter is located in the forward region, outside the field volume. For triggering purposes and to facilitate jet reconstruction, the calorimeter cells are grouped into towers projecting radially outward from the center of the detector. Events are filtered using a two-tier trigger system: a hardware-based first level (L1) and a software-based high-level trigger (HLT). The information from the individual detectors is combined in a global view of the event, the particle-flow (PF) event reconstruction [33,34], which attempts to identify all the particles detected in a collision and to measure their momenta. A more detailed description of the CMS detector, together with a definition of the coordinate system used and the relevant kinematic variables, can be found in Ref. [32].

\section{EVENT SELECTION}

At least one reconstructed vertex is required within $|z|<24 \mathrm{~cm}$. The primary vertex is defined as the vertex with the highest sum of squared transverse momenta for the associated tracks.

The PF algorithm is used to reconstruct the particles in the event and to identify them as muons, electrons (with associated bremsstrahlung photons), photons (unconverted and converted), or charged/neutral hadrons. The PF candidates are clustered into jets using the anti- $k_{\mathrm{T}}$ algorithm [35] with distance parameter of 0.5, implemented in the FASTJET package [36]. An event-by-event jet-area-based correction [37-39] is applied to remove the energy from additional collisions in the same bunch crossing (pileup). The jet momenta are further corrected using calibration constants derived from simulations, test beam results, and pp collision data [39]. All jets in this analysis are required to have transverse momentum $\left(p_{\mathrm{T}}\right)$ greater than $30 \mathrm{GeV}$ and absolute value of pseudorapidity $(\eta)$ less than 2.5 . Jet identification criteria [40] are applied to the two jets in the event with the highest $p_{\mathrm{T}}$ (leading jets), in order to remove spurious events associated with calorimeter noise. The event is rejected if either of these two jets fails these criteria.

Geometrically close jets are combined into "wide jets" $[2,5]$, which are used to measure the dijet mass spectrum and search for new resonances and QBHs. The wide jet algorithm is inspired by studies using jet grooming algorithms [41-43] and is intended to reduce the sensitivity to gluon radiation from the colored final state. The two jets with largest $p_{\mathrm{T}}$ are used as seeds. The Lorentz vectors of all other jets are then added to the closest leading jet, if within $\Delta R=\sqrt{(\Delta \eta)^{2}+(\Delta \phi)^{2}}<1.1$ (where $\phi$ is the azimuthal angle in radians), to obtain two wide jets, which then compose the dijet system. The background from $t$-channel multijet events is suppressed by requiring the pseudorapidity separation of the two wide jets $\left(\left|\Delta \eta_{\mathrm{jj}}\right|\right)$ to be less than 1.3. In addition, we require that both wide jets are reconstructed in the region $|\eta|<2.5$. These requirements maximize the search sensitivity for isotropic decays of dijet resonances in the presence of multijet background [1].

The L1 trigger used for this search requires that the scalar sum of the jet $p_{\mathrm{T}}\left(H_{\mathrm{T}}\right)$ be larger than $150 \mathrm{GeV}$. Events satisfying the L1 trigger are then filtered by the HLT which requires that either of the two following trigger selections is satisfied: the first trigger requires $H_{\mathrm{T}}>650 \mathrm{GeV}$; the second trigger requires that the invariant mass of the dijet system $\left(m_{\mathrm{jj}}\right)$, computed using the same algorithm employed at the reconstruction level, be greater than $750 \mathrm{GeV}$. In the second trigger, $\left|\Delta \eta_{\mathrm{jj}}\right|<1.5$ is required. Biases from the trigger requirements are avoided by requiring that the fully reconstructed events have $m_{\mathrm{jj}}>890 \mathrm{GeV}$. In this region, the combined efficiency of the L1 and HLT triggers is found to be more than $99.7 \%$.

To identify jets originating from the hadronization of $b$ quarks, an algorithm is used that combines information on secondary vertices and reconstructed lifetime. The set of conditions used corresponds to the loose working point of the algorithm, which is known as the combined secondary vertex (CSV) and described in detail in Ref. [44]. The performance is studied using samples of LHC data enriched in or depleted of $b$ quarks, as well as simulated samples. The algorithm is applied to the two leading jets, and events 

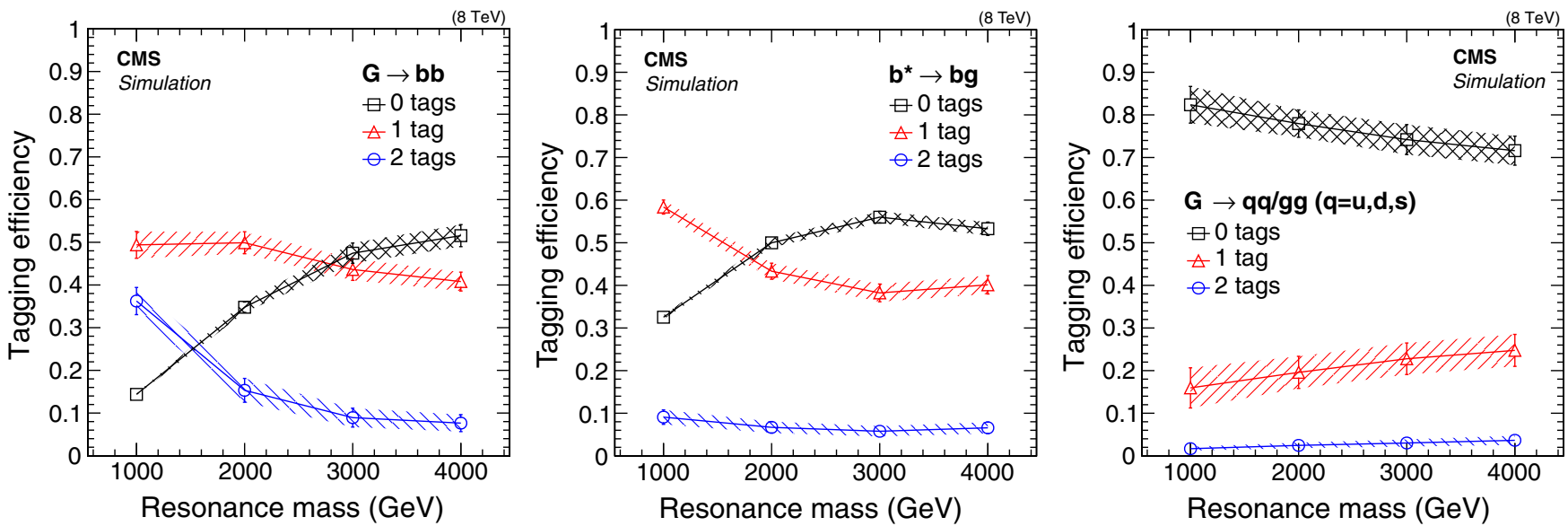

FIG. 1 (color online). Tagging efficiencies for $0 b, 1 b$, and $2 b$ selections as a function of the resonance mass for $b b, b g$, and $q q / g g$ (where $q=u, d, s$ ) decay modes, for a RS graviton $g$ decaying to a $b b$ pair (left), an excited $b^{*}$ quark decaying to a $b$-quark and a gluon (center), and a RS graviton $g$ decaying to two gluons or to a $q q$ pair, with $q=u, d$, or $s$ (right). The hatched regions represent the uncertainties in the tagging efficiencies corresponding to the variation of the $b$-tagging scale factors within their uncertainties.

are categorized as $0 b, 1 b$, or $2 b$. This categorization allows differences in tagging efficiency between data and simulation to be evaluated, as a function of jet $p_{\mathrm{T}}$. Corrections referred to as "b-tagging scale factors" are derived, which are applied to the simulated samples used in the analysis to correct for the differences observed between simulation and data.

The tagging efficiencies for $0 b, 1 b$, and $2 b$ categories are shown in Fig. 1 for RS gravitons and excited $b$ quarks as a function of the resonance mass. The efficiency to tag correctly a $b$-jet decreases as the resonance mass increases. The efficiency of double tagging a resonance that decays into two charm quarks $(\sim 10 \%$ at $1 \mathrm{TeV})$ is systematically higher than that for $g g, q g$, and $q q$ final states with lightflavor quarks (below 5\%), while being significantly lower than for the $b b$ decay mode. To simplify the analysis, charm quarks are assumed to have the same efficiency as light quarks and gluons. This choice translates into weaker sensitivity to $b b$ resonances.

\section{DIJET MASS SPECTRUM}

Figure 2 shows the dijet mass distribution normalized to the integrated luminosity of the sample $\left(d \sigma / d m_{\mathrm{jj}}\right)$ for the inclusive data sample, with bin widths approximately equal to the dijet mass resolution. The data are compared to a leading order (LO) prediction of the multijet background from PYTHIA 6.426 [45] with the $\mathrm{Z2} *$ tune [46] (Z2 is identical to Z1 aside from the choice of the CTEQ6L PDF), where the generated events are processed through a GEANT4-based [47] simulation of the CMS detector.

In the event generation, CTEQ6L1 parton distribution functions (PDF) [48] are used. The renormalization and factorization scales are both set equal to the $p_{\mathrm{T}}$ of the hard-scattered partons. The prediction has been normalized to the data by applying a multiplicative factor of 1.23 . The shape of the PYTHIA prediction agrees with the data within the statistical precision.

A method based on data is used to estimate the background from multijet production. We fit the following parametrization to the data:

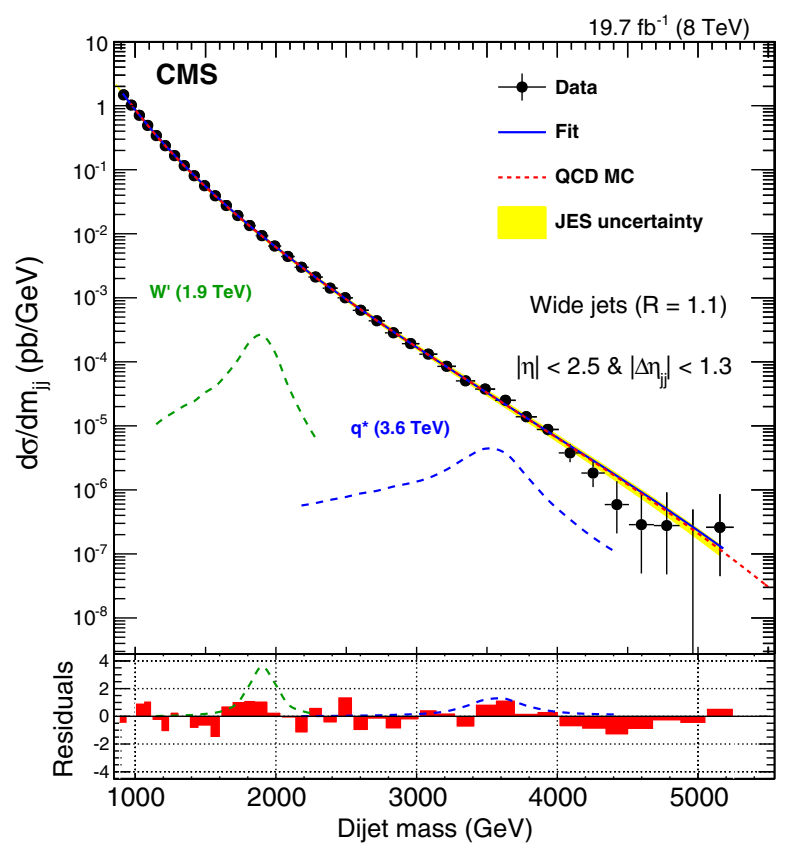

FIG. 2 (color online). Inclusive dijet mass spectrum from wide jets (points) compared to a fit (solid curve) and to predictions including detector simulation of multijet events and signal resonances. The predicted multijet shape (QCD MC) has been scaled to the data (see text). The vertical error bars are statistical only and the horizontal error bars are the bin widths. For comparison,the signal distributions for a $W^{\prime}$ resonance of mass $1900 \mathrm{GeV}$ and an excited quark of mass $3.6 \mathrm{TeV}$ are shown. The bin-by-bin fit residuals scaled to the statistical uncertainty of the data, (data - fit) $/ \sigma_{\text {data }}$, are shown at the bottom and compared with the expected signal contributions. 


$$
\frac{d \sigma}{d m_{\mathrm{jj}}}=\frac{P_{0}(1-x)^{P_{1}}}{x^{P_{2}+P_{3} \ln (x)}}
$$

with the variable $x=m_{\mathrm{jj}} / \sqrt{s}$ and four free parameters $P_{0}$, $P_{1}, P_{2}$, and $P_{3}$. This functional form was used in previous searches $[1,2,5-11,49,50]$ to describe the distribution of both data and multijet background from simulation. A Fisher $F$-test [51] is used to confirm that no additional parameters are needed to model these distributions for a data set as large as the available one. The fit of the data to the function given in Eq. (1) returns a chi-squared value of 26.8 for 35 degrees of freedom. The difference between the data and the fit value is also shown at the bottom of Fig. 2, normalized to the statistical uncertainty of the data. The $0 b, 1 b$, and $2 b m_{\mathrm{jj}}$ dijet mass spectra are shown in Fig. 3. The function of Eq. (1) is also fit to these data
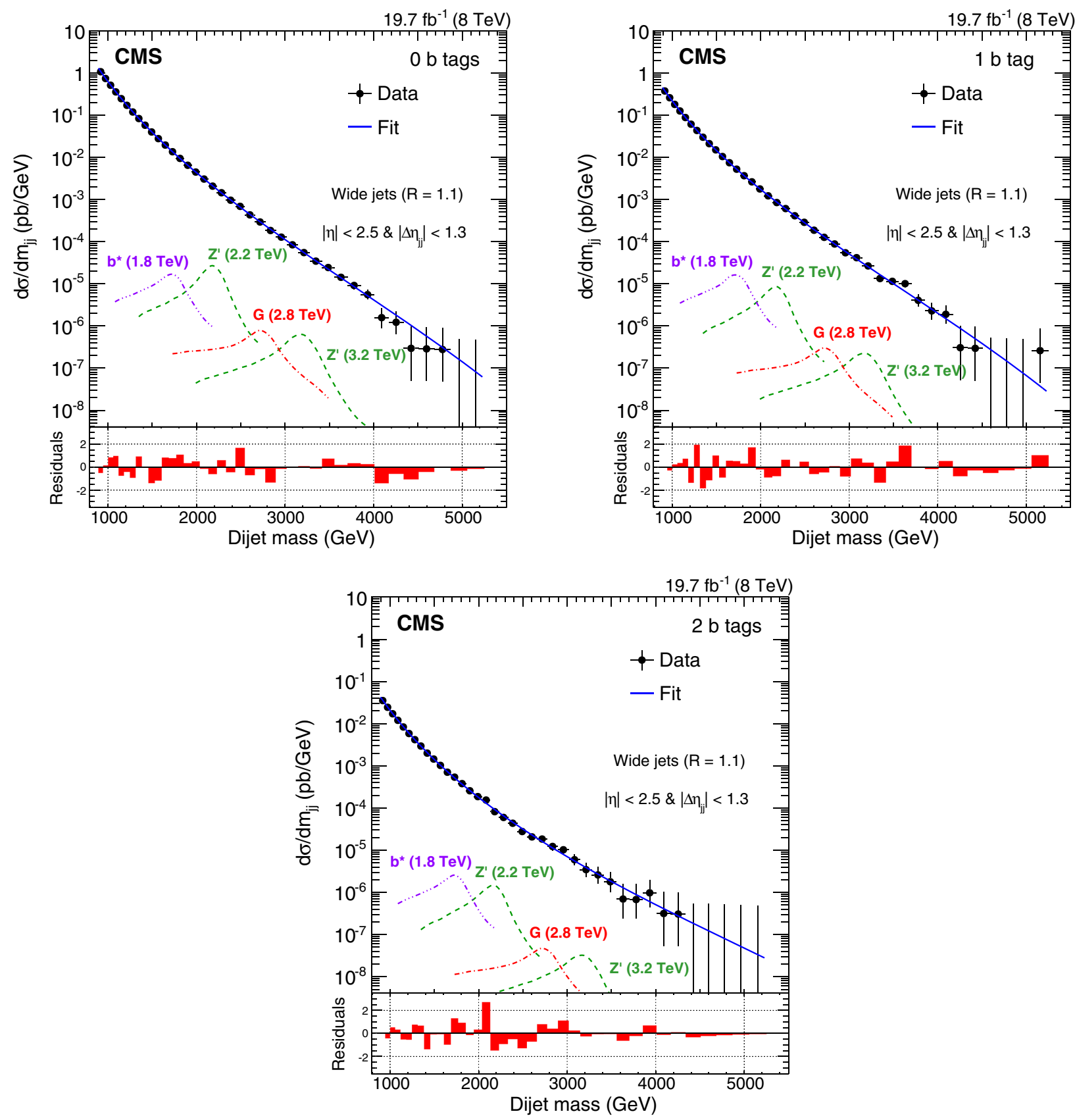

FIG. 3 (color online). Dijet mass spectra (points) in different $b$-tag multiplicity bins compared to a fit (solid curve). The vertical error bars are statistical only and the horizontal error bars are the bin widths. For comparison, signal distributions are shown for an excited $b$ quark of mass $1800 \mathrm{GeV}$, a $Z^{\prime}$ of mass $2200 \mathrm{GeV}$, a RS graviton of mass $2800 \mathrm{GeV}$, and a $Z^{\prime}$ of mass $3200 \mathrm{GeV}$. The bin-by-bin fit residuals scaled to the statistical uncertainty of the data, (data - fit) $/ \sigma_{\text {data }}$, are shown at the bottom of each plot. 
distributions. The data are well described by this function and no significant deviations from the background hypothesis are observed.

For comparison, signal distributions for various narrow resonance models are shown in both Figs. 2 and 3 . These distributions are obtained using PYTHIA 8.153 [52], tune 4C [53], and the CMS detector simulation.

The $q q$ and $g g$ signal shapes are obtained from simulated samples of RS graviton production, respectively $q q \rightarrow G \rightarrow q q$ and $g g \rightarrow G \rightarrow g g$. Graviton decays to all quark flavors other than top are included; the top quark is excluded as its decays do not give rise to the simple dijet experimental signature. The $q g$ signal shapes are obtained from simulations of excited quark production, $q g \rightarrow q^{*} \rightarrow q g$. The simulated samples for the inclusive analysis contain both $u^{*}$ and $d^{*}$ processes, while for the $b$-enriched analysis only $b^{*}$ production is considered. The predicted mass distributions have a
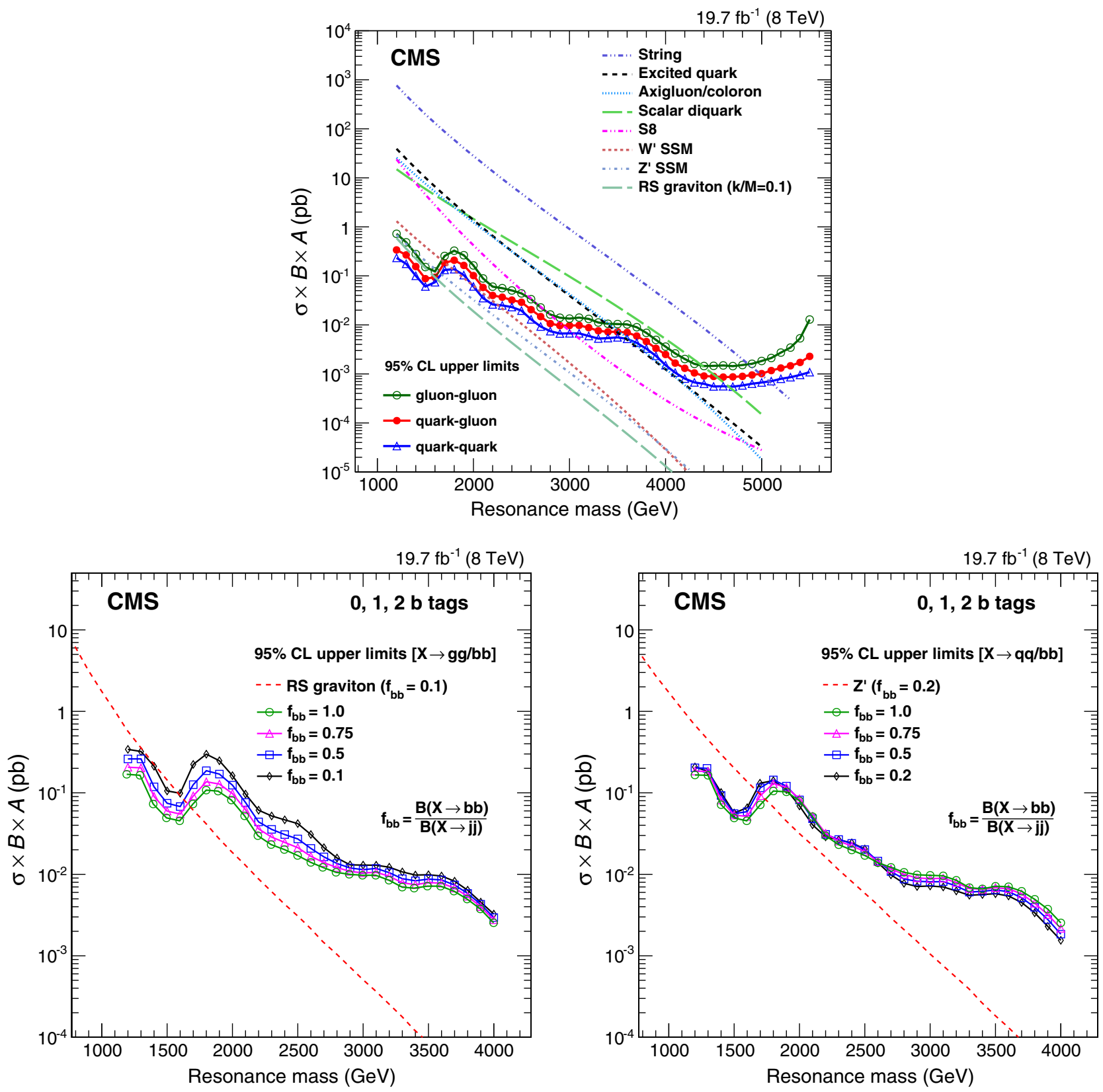

FIG. 4 (color online). The observed 95\% C.L. upper limits on $\sigma \mathcal{B} A$ for narrow dijet resonances. Top: limit on gluon-gluon, quark-gluon, and quark-quark narrow resonances from the inclusive analysis, compared to LO theoretical predictions for string resonances [13,14], excited quarks [16,17], axigluons [18-20], colorons [21], scalar diquarks [15], S8 resonances [22], new SSM gauge bosons $W^{\prime}$ and $Z^{\prime}$ [23], and RS gravitons [24-26]. Bottom left: combined limits on gg/bb resonances for different values of $f_{b b}$. The theoretical cross section for a RS graviton is shown for comparison. Bottom right: combined limits on $q q / b b$ resonances for different values of $f_{b b}$. The theoretical cross section for a $Z^{\prime}$ is shown for comparison. 
Gaussian peak coming from the jet energy resolution (JER) and a tail towards lower mass values induced by the radiation of quarks and gluons at large angles. The contribution of this low mass tail to the line shape depends on the parton content of the resonance ( $q q$, $q g$, or $g g$ ). Resonances containing gluons, which are more susceptible to radiation than quarks, have a more pronounced tail. For high-mass resonances, there is also another significant contribution depending on both parton distributions and the natural width of the BreitWigner resonance shape: when the resonance is produced by interaction of nonvalence partons in the proton, the low-mass component of the Breit-Wigner resonance shape is amplified by a larger parton probability at low fractional momentum, producing a large tail at low-mass values.

\section{INTERPRETATION OF THE RESULTS}

Upper limits are set on the production cross section for different resonance final states $(q q, q g, g g, q q / b b$, $g g / b b$, and $b g)$ as a function of the resonance mass.
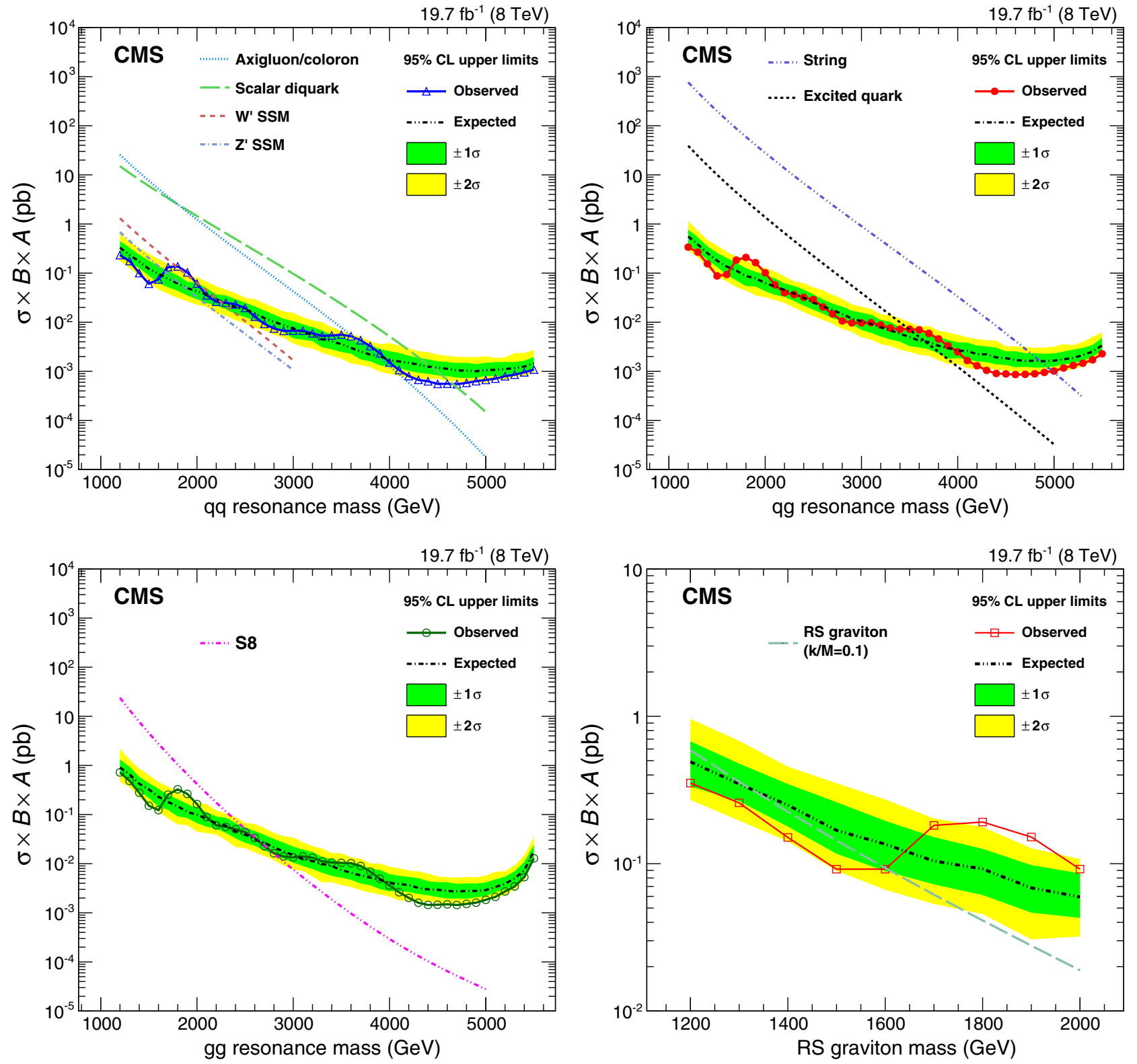

FIG. 5 (color online). The observed 95\% C.L. upper limits on $\sigma \mathcal{B} A$ for narrow resonances decaying into $q q$ (top left), $q g$ (top right), and $g g$ (bottom left) final states, and for RS graviton resonances (bottom right). The limits are shown as points and solid lines. Also shown are the expected limits (dot-dashed dark lines) and their variation at the $1 \sigma$ and $2 \sigma$ levels (shaded bands). Predicted cross sections calculated at LO for various narrow resonances are also shown. 


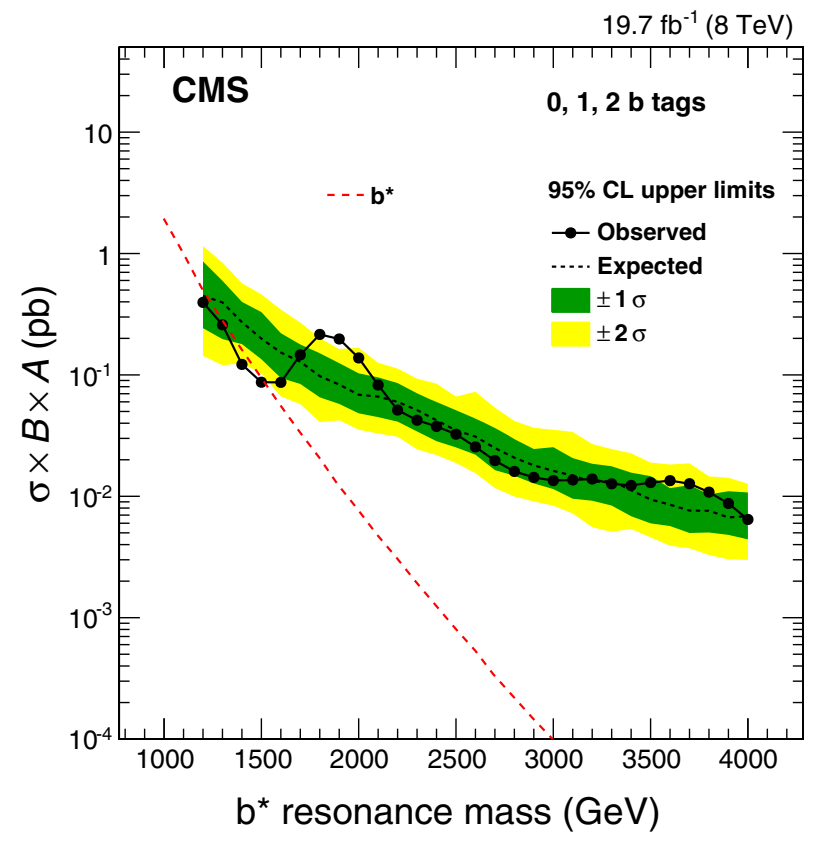

FIG. 6 (color online). Observed and expected 95\% C.L. upper limits on $\sigma \mathcal{B} A$ with systematic uncertainties included, for $b^{*} \rightarrow$ $b g$ resonances, compared with the LO theoretical cross section for excited $b$-quark production.
The limits are computed using a binned likelihood $L$ written as a product of Poisson probability density functions

$$
L=\prod_{i} \frac{\lambda_{i}^{n_{i}} e^{-\lambda_{i}}}{n_{i} !}
$$

where the product runs over the $m_{\mathrm{jj}}$ bins. For the $i$ th $m_{\mathrm{jj}}$ bin, $n_{i}$ is the observed number of events and $\lambda_{i}=\mu N_{i}(S)+$ $N_{i}(B)$ denotes the expected number of events. Here, $N_{i}(B)$ is the expected number of events from multijet background, $N_{i}(S)$ is the expected number of signal events for the benchmark models considered, and $\mu$ the ratio between the signal production cross section and its corresponding benchmark value. The background term $N_{i}(B)$ is estimated using the parametrization of Eq. (1).

The dominant sources of systematic uncertainty are as follows:

(i) uncertainty in the jet energy scale (JES) [39], which translates into a $1 \%$ relative uncertainty in the dijet mass, roughly independent of the mass value; it is propagated to the search by shifting the reconstructed dijet mass for signal events by $\pm 1 \%$;

TABLE I. Observed 95\% C.L. upper limits on $\sigma \mathcal{B} A$ for narrow $q q, q g$, and $g g$ resonances, from the inclusive analysis for signal masses between 1.2 and $5.5 \mathrm{TeV}$.

\begin{tabular}{|c|c|c|c|c|c|c|c|}
\hline \multirow[b]{2}{*}{ Mass (TeV) } & \multicolumn{3}{|c|}{ Upper limit on $\sigma \mathcal{B} A(\mathrm{fb})$} & \multirow[b]{2}{*}{ Mass $(\mathrm{TeV})$} & \multicolumn{3}{|c|}{ Upper limit on $\sigma \mathcal{B} A(\mathrm{fb})$} \\
\hline & $q q$ & $q g$ & $g g$ & & $q q$ & $q g$ & $g g$ \\
\hline 1.2 & 230 & 340 & 720 & 3.4 & 5.4 & 7.3 & 11 \\
\hline 1.3 & 180 & 270 & 480 & 3.5 & 5.6 & 7.2 & 10 \\
\hline 1.4 & 100 & 160 & 280 & 3.6 & 5.2 & 7.0 & 10 \\
\hline 1.5 & 60 & 88 & 150 & 3.7 & 4.3 & 5.9 & 9.0 \\
\hline 1.6 & 74 & 94 & 120 & 3.8 & 3.3 & 4.6 & 6.9 \\
\hline 1.7 & 130 & 180 & 250 & 3.9 & 2.3 & 3.3 & 4.9 \\
\hline 1.8 & 140 & 210 & 330 & 4.0 & 1.5 & 2.5 & 3.6 \\
\hline 1.9 & 100 & 160 & 260 & 4.1 & 1.1 & 1.7 & 2.6 \\
\hline 2.0 & 60 & 100 & 160 & 4.2 & 0.80 & 1.3 & 2.0 \\
\hline 2.1 & 35 & 58 & 89 & 4.3 & 0.67 & 1.0 & 1.6 \\
\hline 2.2 & 26 & 40 & 60 & 4.4 & 0.63 & 0.91 & 1.4 \\
\hline 2.3 & 25 & 37 & 56 & 4.5 & 0.56 & 0.89 & 1.5 \\
\hline 2.4 & 23 & 32 & 50 & 4.6 & 0.56 & 0.86 & 1.5 \\
\hline 2.5 & 20 & 29 & 44 & 4.7 & 0.55 & 0.87 & 1.4 \\
\hline 2.6 & 13 & 20 & 33 & 4.8 & 0.58 & 0.89 & 1.5 \\
\hline 2.7 & 9.3 & 15 & 23 & 4.9 & 0.63 & 0.95 & 1.6 \\
\hline 2.8 & 7.4 & 11 & 16 & 5.0 & 0.67 & 1.0 & 1.8 \\
\hline 2.9 & 6.7 & 9.7 & 14 & 5.1 & 0.72 & 1.2 & 2.1 \\
\hline 3.0 & 6.7 & 9.7 & 13 & 5.2 & 0.80 & 1.3 & 2.7 \\
\hline 3.1 & 6.7 & 9.8 & 14 & 5.3 & 0.86 & 1.5 & 3.4 \\
\hline 3.2 & 5.9 & 8.8 & 13 & 5.4 & 0.95 & 1.7 & 5.3 \\
\hline 3.3 & 5.3 & 7.6 & 11 & 5.5 & 1.1 & 2.3 & 13 \\
\hline
\end{tabular}


(ii) uncertainty in the JER [39], which translates into an uncertainty of $10 \%$ in the dijet mass resolution [39]. This uncertainty is propagated to the search by increasing and decreasing by $10 \%$ the reconstructed width of the dijet mass shape for the signal;

(iii) the precision in the overall normalization for the signal is limited by an uncertainty of $2.6 \%$ in the integrated luminosity [54];

(iv) $b$-tagging scale factors ( $\sim 5 \%$ for heavy and $\sim 10 \%$ for light-flavor jets) [44], applied only in the dedicated $b$-jet search.

(v) uncertainties due to the choice of the background fit function are taken into account by the marginalization procedure described below.

Using studies based on simulations, the dependence of the signal mass shapes on the number of pileup interactions is found to be negligible. Similarly, no appreciable difference in the signal acceptance is observed when different PDF sets are used.

For setting upper limits on signal cross sections a Bayesian formalism [55] is used, with a uniform prior for the signal cross section in the range $[0,+\infty]$. For a given value of the resonance mass the data are fit to the background function plus a signal line shape, the signal cross section being a free parameter. The resulting fit function with the signal cross section set to zero is used as the initial background hypothesis. The uncertainty in the background shape is incorporated by marginalizing over the background-fit parameters using uniform priors. The integration is performed in a sufficiently large range around the best-fit values such that the results are found to be stable. Uncertainties due to alternative background fit functions are not explicitly included since these variations are already covered by the marginalization procedure with the default fit function [Eq. (1)]. Log-normal priors are

TABLE II. Observed $95 \%$ C.L. upper limits on $\sigma \mathcal{B} A$ for narrow $g g / b b, q q / b b$, and $b g$ resonances from the $b$-enriched analysis, for signal masses between 1.2 and $4.0 \mathrm{TeV}$. The upper limits are given for different ratios $f_{b b}$ for $g g / b b$ and $q q / b b$ resonances, and for $100 \%$ branching fraction into $\mathrm{bg}$.

\begin{tabular}{|c|c|c|c|c|c|c|c|c|c|}
\hline \multirow[b]{3}{*}{ Mass $(\mathrm{TeV})$} & \multicolumn{9}{|c|}{ Upper limit on $\sigma \mathcal{B} A(\mathrm{fb})$} \\
\hline & \multicolumn{4}{|c|}{$g g / b b$} & \multicolumn{4}{|c|}{$q q / b b$} & \multirow[t]{2}{*}{$b g$} \\
\hline & $f_{b b}=0.2$ & $f_{b b}=0.5$ & $f_{b b}=0.75$ & $f_{b b}=1.0$ & $f_{b b}=0.2$ & $f_{b b}=0.5$ & $f_{b b}=0.75$ & $f_{b b}=1.0$ & \\
\hline 1.2 & 340 & 260 & 210 & 170 & 210 & 200 & 180 & 170 & 400 \\
\hline 1.3 & 320 & 260 & 200 & 160 & 180 & 200 & 180 & 160 & 260 \\
\hline 1.4 & 210 & 120 & 90 & 72 & 100 & 93 & 82 & 72 & 120 \\
\hline 1.5 & 110 & 75 & 60 & 49 & 57 & 56 & 53 & 49 & 87 \\
\hline 1.6 & 99 & 68 & 55 & 45 & 66 & 59 & 51 & 45 & 87 \\
\hline 1.7 & 220 & 130 & 92 & 71 & 130 & 120 & 92 & 71 & 150 \\
\hline 1.8 & 300 & 190 & 140 & 110 & 140 & 140 & 130 & 110 & 220 \\
\hline 1.9 & 250 & 170 & 130 & 100 & 110 & 120 & 120 & 100 & 200 \\
\hline 2.0 & 160 & 120 & 98 & 80 & 69 & 82 & 85 & 80 & 140 \\
\hline 2.1 & 97 & 76 & 62 & 51 & 41 & 48 & 52 & 51 & 83 \\
\hline 2.2 & 61 & 44 & 36 & 30 & 29 & 31 & 32 & 30 & 51 \\
\hline 2.3 & 52 & 36 & 29 & 23 & 27 & 27 & 26 & 23 & 42 \\
\hline 2.4 & 47 & 31 & 25 & 20 & 24 & 24 & 22 & 20 & 38 \\
\hline 2.5 & 42 & 27 & 21 & 17 & 20 & 20 & 19 & 17 & 32 \\
\hline 2.6 & 31 & 21 & 17 & 14 & 14 & 15 & 14 & 14 & 26 \\
\hline 2.7 & 21 & 16 & 14 & 12 & 9.9 & 11 & 12 & 12 & 20 \\
\hline 2.8 & 16 & 14 & 12 & 11 & 7.8 & 8.8 & 10 & 11 & 16 \\
\hline 2.9 & 13 & 12 & 11 & 9.9 & 7.1 & 8.2 & 9.0 & 9.9 & 14 \\
\hline 3.0 & 13 & 11 & 10 & 9.7 & 7.2 & 8.1 & 8.8 & 9.7 & 13 \\
\hline 3.1 & 13 & 12 & 11 & 9.6 & 7.0 & 8.1 & 9.0 & 9.6 & 14 \\
\hline 3.2 & 12 & 10 & 9.5 & 8.4 & 6.3 & 7.2 & 7.9 & 8.4 & 14 \\
\hline 3.3 & 11 & 8.8 & 7.9 & 6.9 & 5.5 & 6.0 & 6.7 & 6.9 & 13 \\
\hline 3.4 & 9.8 & 8.3 & 7.5 & 6.6 & 5.7 & 6.2 & 6.6 & 6.6 & 12 \\
\hline 3.5 & 9.9 & 8.7 & 7.9 & 7.1 & 5.8 & 6.4 & 6.8 & 7.1 & 13 \\
\hline 3.6 & 9.5 & 8.5 & 7.8 & 7.0 & 5.4 & 6.1 & 6.6 & 7.0 & 13 \\
\hline 3.7 & 8.2 & 7.4 & 6.8 & 6.2 & 4.5 & 5.2 & 5.7 & 6.2 & 13 \\
\hline 3.8 & 6.4 & 5.8 & 5.4 & 4.9 & 3.4 & 3.9 & 4.4 & 4.9 & 11 \\
\hline 3.9 & 4.6 & 4.3 & 4.0 & 3.7 & 2.3 & 2.8 & 3.2 & 3.7 & 8.7 \\
\hline 4.0 & 3.2 & 2.9 & 2.8 & 2.5 & 1.5 & 1.8 & 2.2 & 2.5 & 6.4 \\
\hline
\end{tabular}


used to model systematic uncertainties in the JES, JER, integrated luminosity, and $b$-tagging efficiency, all treated as nuisance parameters. The nuisance parameters are marginalized to derive a posterior probability density function for the signal cross section. The marginalization is performed using Markov chain Monte Carlo integration implemented in the Bayesian Analysis Toolkit [56].

In the case of the search for $X \rightarrow b b$ resonances the limit is obtained by combining the three event categories $(0 b, 1 b$, and $2 b$ ). The background distributions in the three samples
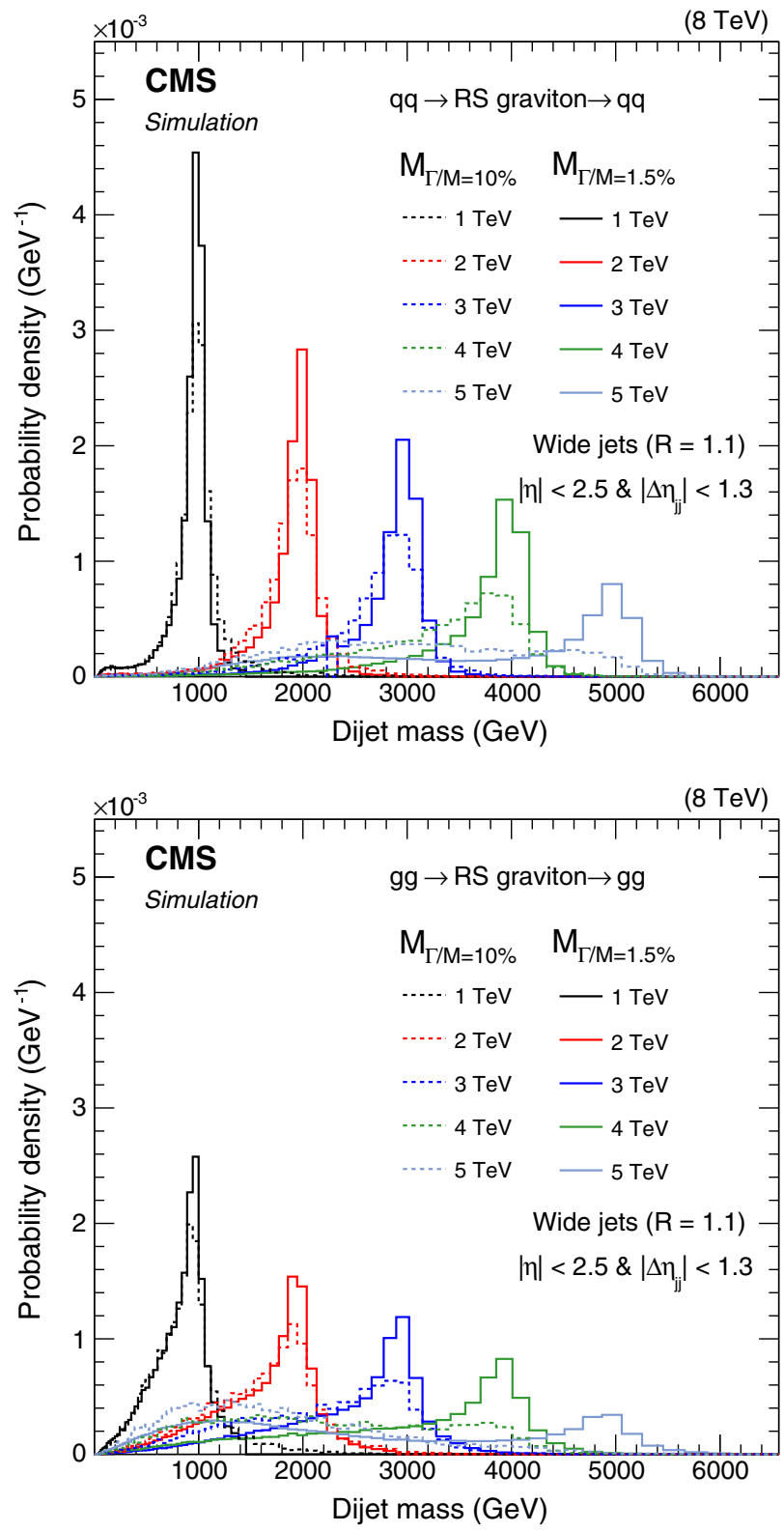

FIG. 7 (color online). Dijet mass distributions for $q q$ (left) and $g g$ (right) resonances with masses of $1,2,3,4$, and $5 \mathrm{TeV}$ and two different values of $\Gamma / M(10 \%$ and $1.5 \%)$. The corrections for the difference in the JES between a parametric simulation and the GEANT4 -based CMS simulation have been applied. are independently varied in the fit. The relative normalization of the signal distributions in the three samples is determined by the ratio of the branching fractions of the $X$ resonance:

$$
f_{b b}=\frac{\mathcal{B}(X \rightarrow b b)}{\mathcal{B}(X \rightarrow \mathrm{jj})} .
$$

As the fraction increases, events from a resonance in the $0 b$ category shift into the $1 b$ and $2 b$ categories. The distribution between the three categories also depends on the tagging efficiencies shown in Fig. 1. Mistags of light-flavor jets are accounted for, according to the quoted tagging probabilities.

Figure 4 shows the observed model independent upper limits at the 95\% confidence level (CL) on the product of the cross section $(\sigma)$, the branching fraction into dijets $(b)$, and the acceptance $(A)$ for the kinematic requirements described in Sec. III, for narrow $q q, g$, and $g g$ resonances. The acceptance for isotropic decays is $A \approx 0.6$, independent of resonance mass. The observed upper limits can be compared to LO predictions for $\sigma \mathcal{B A}$ at the parton level, without any detector simulation, in order to determine mass limits on new particles. The two partons in the LO process of the resonance decay should both have pseudorapidity

TABLE III. Observed and expected 95\% C.L. exclusions on the mass of various resonances. Systematic uncertainties are taken into account. For excited $b$ quark the expected mass limit is below the range of this analysis. For the axigluon/coloron and coloroctet scalar only observed mass limits are computed.

\begin{tabular}{|c|c|c|c|}
\hline \multicolumn{4}{|c|}{ Inclusive search } \\
\hline Model & $\begin{array}{l}\text { Final } \\
\text { state }\end{array}$ & $\begin{array}{l}\text { Observed mass } \\
\text { exclusion }(\mathrm{TeV})\end{array}$ & $\begin{array}{l}\text { Expected mass } \\
\text { exclusion }(\mathrm{TeV})\end{array}$ \\
\hline $\begin{array}{l}\text { String } \\
\quad \text { resonance }(S)\end{array}$ & $q g$ & {$[1.2,5.0]$} & {$[1.2,4.9]$} \\
\hline $\begin{array}{l}\text { Excited } \\
\quad \text { quark }\left(q^{*}\right)\end{array}$ & $q g$ & {$[1.2,3.5]$} & {$[1.2,3.7]$} \\
\hline$E_{6}$ diquark (D) & $q q$ & {$[1.2,4.7]$} & {$[1.2,4.4]$} \\
\hline$W^{\prime}$ boson $\left(W^{\prime}\right)$ & $q \bar{q}$ & $\begin{array}{r}{[1.2,1.9]+} \\
{[2.0,2.2]}\end{array}$ & {$[1.2,2.2]$} \\
\hline$Z^{\prime}$ boson $\left(Z^{\prime}\right)$ & $q \bar{q}$ & {$[1.2,1.7]$} & {$[1.2,1.8]$} \\
\hline $\begin{array}{c}\text { RS graviton }(G), \\
k / \bar{M}_{\mathrm{Pl}}=0.1\end{array}$ & $q \bar{q}+g g$ & {$[1.2,1.6]$} & {$[1.2,1.3]$} \\
\hline \multicolumn{4}{|c|}{$b$-enriched search } \\
\hline $\begin{array}{l}\text { Excited } b \\
\quad \text { quark }\left(b^{*}\right)\end{array}$ & $b g$ & {$[1.2,1.6]$} & \\
\hline \multicolumn{4}{|c|}{ Wide resonance search } \\
\hline $\begin{array}{l}\text { Axigluon } \\
\text { (A)/coloron (C) }\end{array}$ & $q \bar{q}$ & {$[1.3,3.6]$} & \\
\hline $\begin{array}{l}\text { Color-octet } \\
\text { scalar (S8) }\end{array}$ & $g g$ & {$[1.3,2.5]$} & \\
\hline
\end{tabular}


less than 2.5 , their pseudorapidity separation should be less than 1.3, and their combined invariant mass should exceed $890 \mathrm{GeV}$. The results shown are obtained in the narrow-width approximation using CTEQ6L1 parton distributions [48].

The expected limits on the cross section are estimated with pseudoexperiments generated using background shapes, which are obtained by signal-plus-background fits to the data. Figure 5 shows the expected limits and their uncertainty bands for $q q, q g$, and $g g$ resonances compared to both observed limits and model predictions. For the RS graviton, which couples either to a pair of gluons or to a $q q$ pair, the model-dependent cross section limits are obtained using a weighted average of the $q q$ (where $q=u, d, c, s$, and $b$, excluding the top quark) and $g g$ dijet mass shapes. The weight factors of about 0.5 correspond to the relative branching fractions for these two final states derived from Ref. [26]. Figure 6 shows a similar plot for an excited $b$ quark. The observed upper limits are reported in Tables I and II for the inclusive and $b$-enriched analyses, respectively. The limits for resonances with gluons in the final states are less restrictive than those with quarks because the signal shapes are wider, as shown for example in Fig. 7.

New particles are excluded at 95\% C.L. in mass regions for which the theoretical curve lies above the observed upper limit for the appropriate final state. The observed and expected mass exclusions for various models are reported in Table III. Table III also shows limits on axigluons/colorons and S8 resonances, interpreted as wide resonances, as discussed in the next section. For comparison with previous searches, we quote here the new limits at a 95\% C.L. on these two models interpreted as narrow resonances, as shown in Fig. 5. These limits provide reference values to quantify the impact of a non-negligible resonance width. For narrow axigluons/ colorons the observed and expected mass limits are 3.7 and $3.9 \mathrm{TeV}$, respectively. The corresponding exclusion limits for narrow S8 resonances are 2.7 and $2.6 \mathrm{TeV}$, respectively.

\section{IMPLICATIONS FOR WIDE RESONANCES}

In the previous sections we have described a search for narrow dijet resonances, where the intrinsic resonance width is negligible compared to the experimental dijet mass resolution. In order to quantify the impact of this search on models with wide resonances, we consider the case of a RS graviton produced via $q q$ and $g g$ annihilation and decaying, respectively, to $q q$ and $g g$ final states. Samples are generated with PYTHIA scanning the plane defined by the graviton mass $M$ and the coupling parameter $k / \bar{M}_{\mathrm{Pl}}$. For resonances with mass at the $\mathrm{TeV}$ scale, the width-to-mass ratio of the resonance is $\Gamma / M \approx 1.4\left(k / \bar{M}_{\mathrm{Pl}}\right)^{2}$ [26]. The excluded signal cross section is presented as a function of the resonance mass and width, separately for the $q q$ and $g g$ final states, in order to allow for the interpretation of the results in a generic model.

The $\left(M, k / \bar{M}_{\mathrm{Pl}}\right)$ scan is performed using events generated with PYTHIA 8.153 and a parametric, fast simulation of the CMS detector [57]. The predicted signal distribution is corrected for the difference in the JES between the fast simulation and the GEANT4-based CMS simulation. Figure 7 shows the corrected dijet mass distributions for several different values of resonance mass $M$ and width-tomass ratio $\Gamma / M$. The excluded cross section at $95 \%$ C.L. as
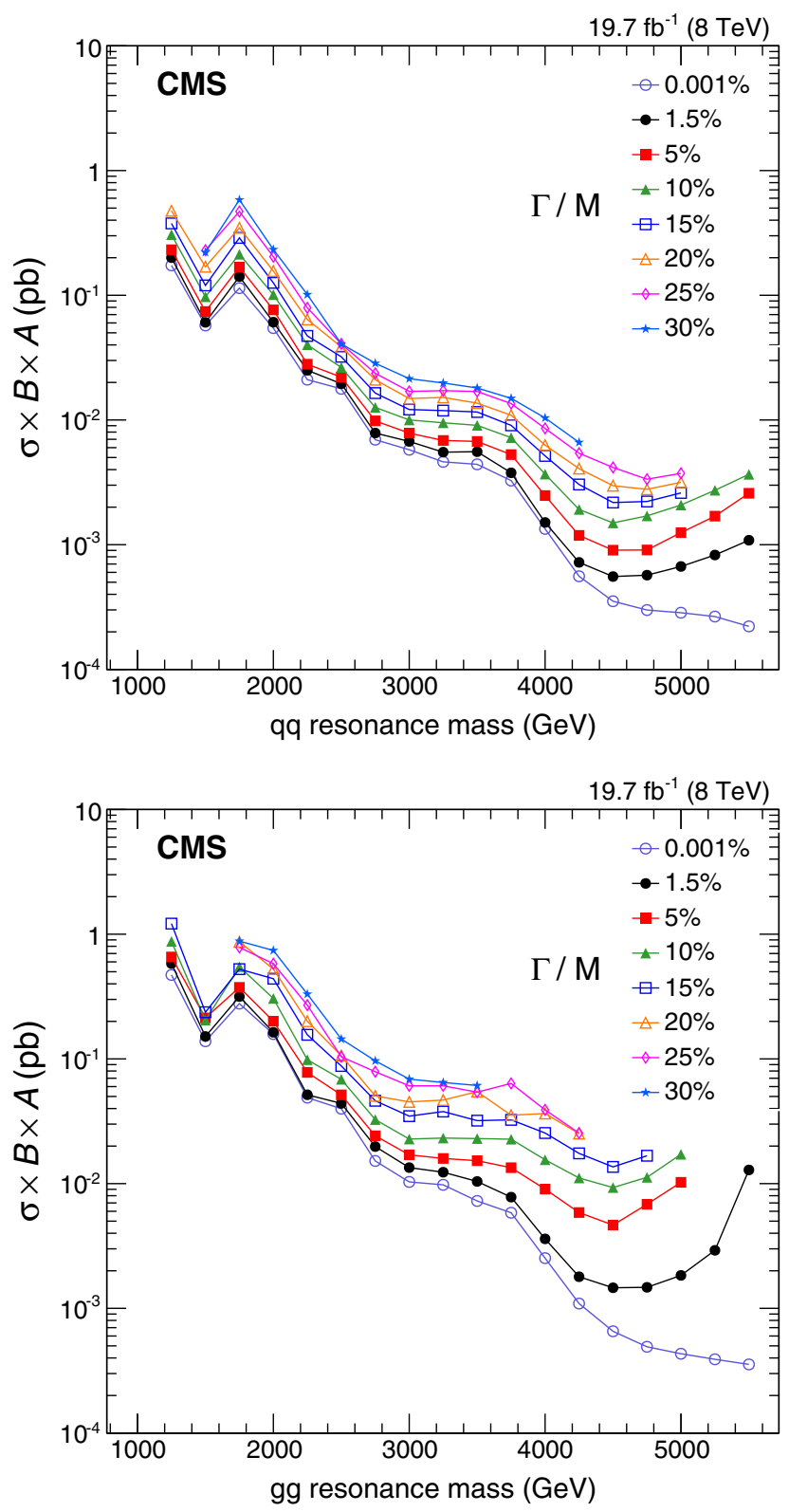

FIG. 8 (color online). Observed 95\% C.L. upper limits on $\sigma \mathcal{B A}$ as a function of the resonance mass for different values of the width-to-mass ratio $\Gamma / M$, computed for $q q \rightarrow G \rightarrow q q$ (left) and $g g \rightarrow G \rightarrow g g$ (right). 
a function of the resonance mass is shown in Fig. 8 for different values of $\Gamma / M$. At resonance masses around 1-2 TeV, the value of the excluded cross section slightly increases with the resonance width, as expected from the gradual widening of the core of the resonance approximately independent of the tail. For large resonance masses, the exclusion limit for wide resonances is worse than the narrow resonance limits by at least one order of magnitude. This different behavior is caused by the enhancement in the low-mass tail of the dijet mass signal shape from partons with low fractional momentum, which is more important for high-mass resonances. Nevertheless, the analysis

TABLE IV. Observed 95\% C.L. upper limits on $\sigma \mathcal{B} A$ as a function of resonance mass for several values of the width-to-mass ratio $\Gamma / M$, computed for $q q \rightarrow G \rightarrow q q$ and $g g \rightarrow G \rightarrow g g$. The missing entries correspond to the region where the two conditions for the validity of the wide resonance analysis are not satisfied (see text).

\begin{tabular}{|c|c|c|c|c|c|c|c|c|}
\hline \multicolumn{9}{|c|}{ Observed $95 \%$ C.L. $\sigma \mathcal{B} A$ limit (fb) } \\
\hline & \multicolumn{8}{|c|}{$\Gamma / M(\%)$ for $q q \rightarrow G \rightarrow q q$} \\
\hline Mass (TeV) & 0.001 & 1.5 & 5 & 10 & 15 & 20 & 25 & 30 \\
\hline 1.25 & 170 & 200 & 230 & 310 & 380 & 470 & & \\
\hline 1.50 & 57 & 61 & 74 & 97 & 120 & 170 & 230 & 220 \\
\hline 1.75 & 110 & 140 & 170 & 210 & 290 & 350 & 470 & 580 \\
\hline 2.00 & 55 & 61 & 76 & 100 & 130 & 160 & 200 & 230 \\
\hline 2.25 & 21 & 25 & 28 & 40 & 47 & 64 & 80 & 100 \\
\hline 2.50 & 18 & 20 & 22 & 26 & 32 & 39 & 41 & 41 \\
\hline 2.75 & 6.9 & 7.9 & 9.8 & 13 & 16 & 21 & 24 & 28 \\
\hline 3.00 & 5.8 & 6.7 & 7.8 & 10 & 12 & 15 & 17 & 21 \\
\hline 3.25 & 4.6 & 5.5 & 6.9 & 9.5 & 12 & 15 & 17 & 20 \\
\hline 3.50 & 4.4 & 5.6 & 6.7 & 9.1 & 12 & 14 & 17 & 18 \\
\hline 3.75 & 3.2 & 3.8 & 5.3 & 7.2 & 9.1 & 11 & 14 & 15 \\
\hline 4.00 & 1.3 & 1.5 & 2.5 & 3.7 & 5.2 & 6.3 & 8.6 & 10 \\
\hline 4.25 & 0.56 & 0.72 & 1.2 & 1.9 & 3.0 & 4.1 & 5.4 & 6.6 \\
\hline 4.50 & 0.35 & 0.56 & 0.90 & 1.5 & 2.2 & 3.0 & 4.2 & \\
\hline 4.75 & 0.30 & 0.57 & 0.91 & 1.7 & 2.2 & 2.8 & 3.3 & \\
\hline 5.00 & 0.28 & 0.67 & 1.2 & 2.1 & 2.6 & 3.2 & 3.7 & \\
\hline 5.25 & 0.26 & 0.83 & 1.7 & 2.7 & & & & \\
\hline 5.50 & 0.22 & 1.1 & 2.6 & 3.7 & & & & \\
\hline \multicolumn{9}{|c|}{ Observed $95 \%$ C.L. $\sigma \mathcal{B} A$ limit (fb) } \\
\hline & \multicolumn{8}{|c|}{$\Gamma / M(\%)$ for $g g \rightarrow G \rightarrow g g$} \\
\hline Mass (TeV) & 0.001 & 1.5 & 5 & 10 & 15 & 20 & 25 & 30 \\
\hline 1.25 & 470 & 580 & 660 & 880 & 1200 & & & \\
\hline 1.50 & 140 & 150 & 210 & 200 & 240 & & & \\
\hline 1.75 & 280 & 320 & 370 & 550 & 520 & 870 & 780 & 880 \\
\hline 2.00 & 160 & 160 & 200 & 310 & 440 & 530 & 580 & 740 \\
\hline 2.25 & 49 & 52 & 78 & 99 & 160 & 200 & 270 & 330 \\
\hline 2.50 & 40 & 44 & 51 & 69 & 88 & 110 & 100 & 140 \\
\hline 2.75 & 15 & 20 & 24 & 33 & 46 & 50 & 79 & 97 \\
\hline 3.00 & 10 & 13 & 17 & 23 & 35 & 45 & 61 & 69 \\
\hline 3.25 & 9.8 & 12 & 16 & 23 & 38 & 47 & 61 & 65 \\
\hline 3.50 & 7.2 & 10 & 15 & 23 & 32 & 55 & 54 & 61 \\
\hline 3.75 & 5.8 & 7.8 & 13 & 23 & 32 & 35 & 64 & \\
\hline 4.00 & 2.5 & 3.6 & 9.0 & 16 & 25 & 36 & 40 & \\
\hline 4.25 & 1.1 & 1.8 & 5.9 & 11 & 17 & 25 & 25 & \\
\hline 4.50 & 0.65 & 1.5 & 4.7 & 9.3 & 14 & & & \\
\hline 4.75 & 0.49 & 1.5 & 6.8 & 11 & 17 & & & \\
\hline 5.00 & 0.43 & 1.8 & 10 & 17 & & & & \\
\hline 5.25 & 0.39 & 2.9 & & & & & & \\
\hline 5.50 & 0.36 & 1.3 & & & & & & \\
\hline
\end{tabular}


remains sensitive to new resonances up to $\Gamma / M \approx 30 \%$. The cross section limits are reported in Table IV for $q q$ and $g g$ final states. The limits are quoted for a range of masses and widths that satisfies two conditions: (i) at low resonance mass, the core of the signal shape is preserved after the trigger selection $m_{\mathrm{jj}}>890 \mathrm{GeV}$, (ii) at high resonance mass, the presence of the low-mass tails in the signal shape
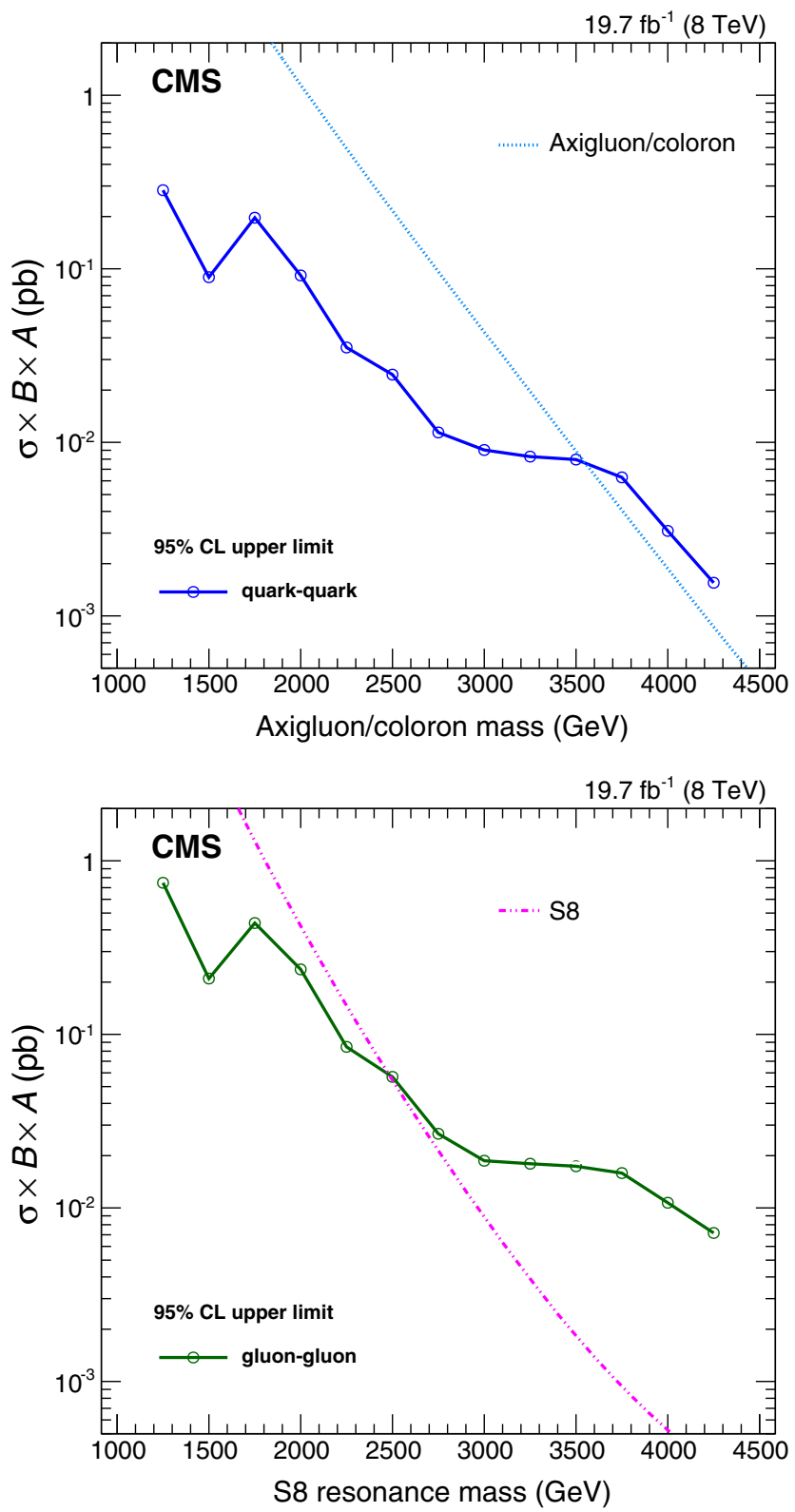

FIG. 9 (color online). Observed 95\% C.L. upper limits on $\sigma \mathcal{B A}$ with systematic uncertainties included for axigluon/coloron (left) and S8 (right) wide resonances, compared to the corresponding theoretical predictions. The axigluon/coloron and $\mathrm{S} 8$ resonances have a relative width $\Gamma / M$ between $5 \%$ and $10 \%$. More details on the cross section calculations for wide resonances are reported in the Appendix. does not significantly affect the limit value. This latter condition is enforced by requiring that the expected limit derived for a truncated signal shape is close to that derived for the full shape, within the typical uncertainty of $30 \%$ in the expected limits. The truncated shape is cut off at $85 \%$ of the nominal resonance mass, and the corresponding limit corrected for the difference in acceptance because of the truncation.

We present below an example, illustrating how to use these generic upper limits on the cross section to set lower mass limits for specific models of wide resonances. The axigluon/coloron and S8 resonances represent good benchmark models for this study, having relative widths $\Gamma / M$ equal to $\alpha_{S}$ and $5 / 6 \alpha_{S}$, respectively (where $\alpha_{S}$ is the SM strong coupling evaluated at an energy scale equal to the resonance mass). $\Gamma / M$ is therefore between $5 \%$ and $10 \%$, slightly decreasing with the increase in the resonance mass because of the running of the strong coupling constant. New cross section upper limits for axigluon/ coloron and S8 resonances are produced, which are, respectively, a linear interpolation between the $\Gamma / M=$ $5 \%$ and $10 \% q q$ and $g g$ limits reported in Table IV. The resulting cross section upper limits are shown in Fig. 9 where they are compared to theoretical predictions to extract the lower mass limits on axigluon/coloron and S8 resonances reported in Table III. More details on the cross section calculations for wide resonances are reported in the Appendix.

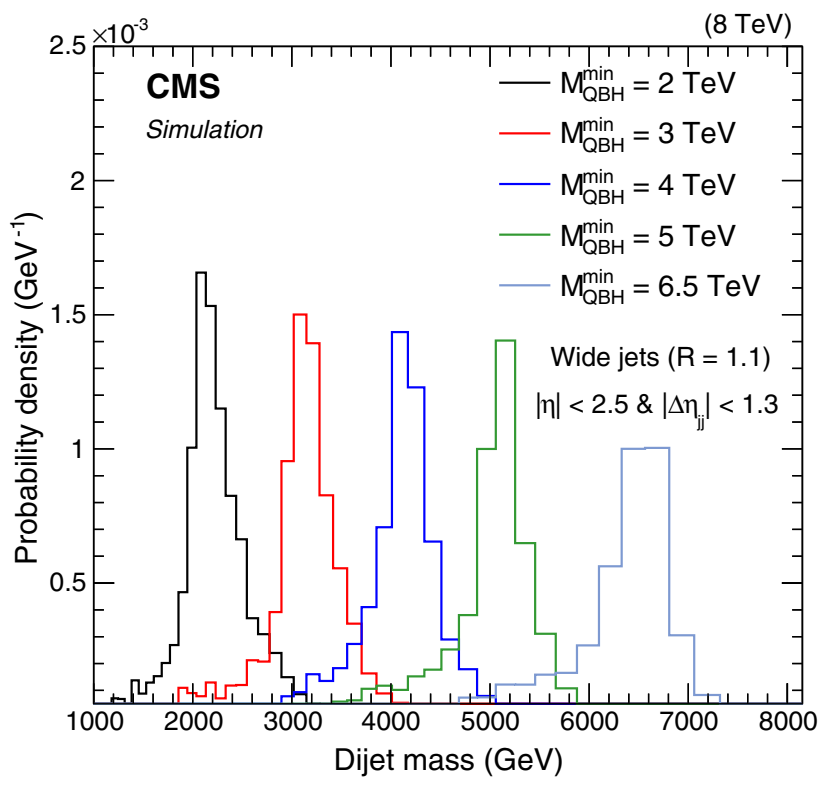

FIG. 10 (color online). Dijet mass distribution for QBHs with $M_{\mathrm{QBH}}^{\min }$ from 2 to $6.5 \mathrm{TeV}$. The signal shape is almost independent both of the number of extra dimensions $n$ and the scale $M_{\mathrm{D}}$. 


\section{IMPLICATIONS FOR QUANTUM BLACK HOLES}

The inclusive dijet search can be interpreted in terms of QBH production [27-29] in models with large $(n \geq 2)$ or warped $(n=1)$ dimensions, where $n$ is the number of extra dimensions. The dijet invariant mass distribution expected from QBH decays is used here, in place of the resonance line shape employed in the other analyses. The required mass shapes are modeled using the QBH (v1.07) matrix-element generator [58] with the CTEQ6L1 PDF set
[48], followed by the parton showering simulation with PYTHIA 8 and a parametric, fast simulation of the CMS detector [57]. The signal is characterized by a peak in the reconstructed dijet mass spectrum, as shown in Fig. 10. The peak position is related to the minimum mass of QBHs, $M_{\mathrm{QBH}}^{\min }$. The relatively narrow shape is a consequence of the convolution of the thresholdlike production behavior for QBHs with the steeply falling parton luminosity as a function of the subprocess center-of-mass energy. The low-mass dijet tails are due to detector resolution effects.
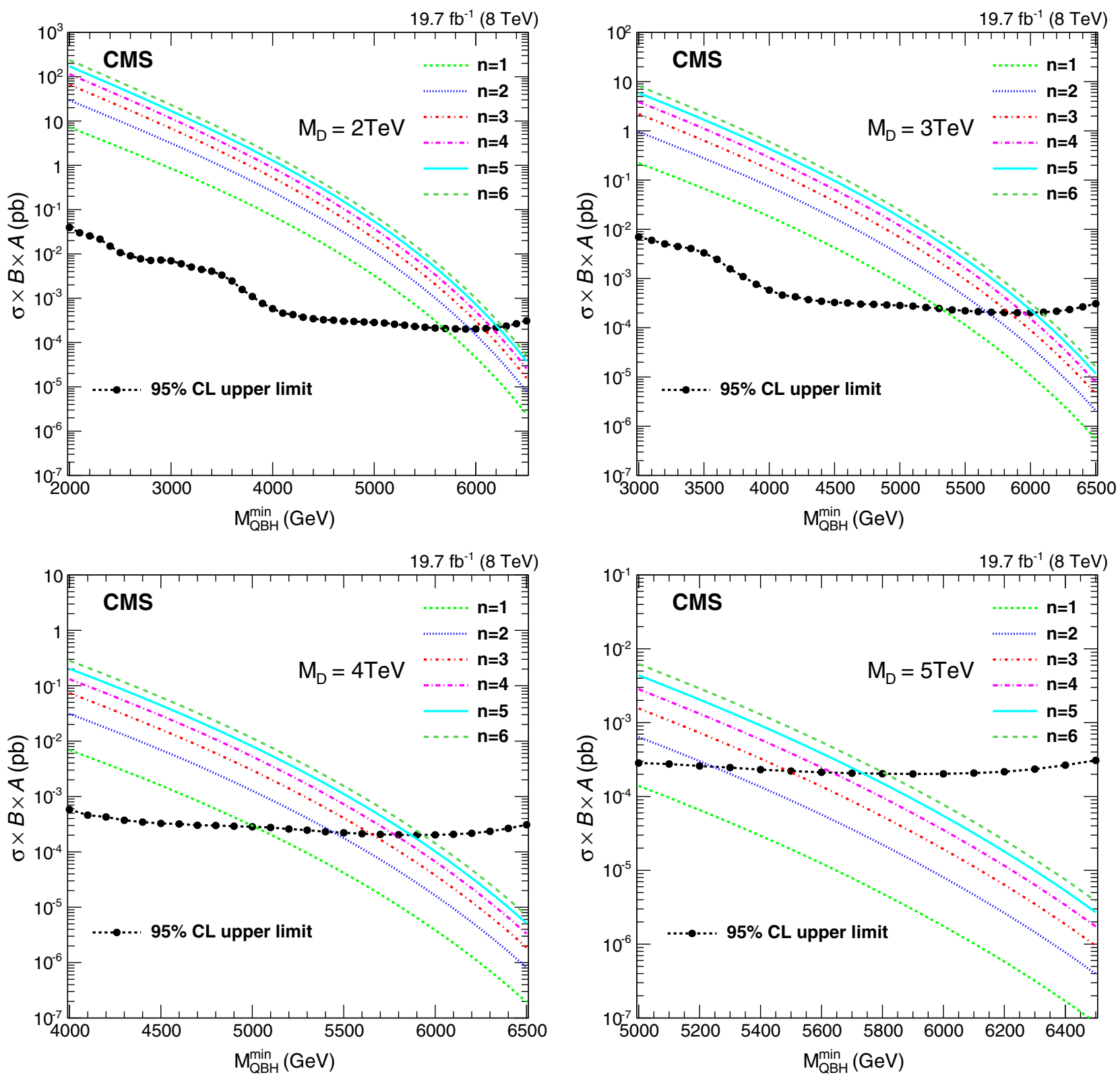

FIG. 11 (color online). Observed 95\% C.L. upper limits on $\sigma \mathcal{B} A$ as a function of $M_{\mathrm{QBH}}^{\mathrm{min}}$, compared to theoretical predictions for different values of the fundamental Planck scale, $M_{\mathrm{D}}$, of $2 \mathrm{TeV}$ (top left), $3 \mathrm{TeV}$ (top right), $4 \mathrm{TeV}$ (bottom left), and $5 \mathrm{TeV}$ (bottom right), with the number of extra dimensions $n$ ranging from 1 to 6 . 
TABLE V. Observed 95\% C.L. upper limits on $\sigma \mathcal{B} A$ for QBHs from the inclusive analysis. These limits are valid for the number of extra dimensions $n$ considered in this paper, ranging from 1 to 6 . Cross section limits are presented only for $M_{\mathrm{QBH}}^{\mathrm{min}} \geq M_{\mathrm{D}}$ for different values of $M_{\mathrm{D}}$, as described in the text.

\begin{tabular}{|c|c|c|c|c|c|}
\hline \multirow[b]{2}{*}{$M_{\mathrm{QBH}}^{\min }(\mathrm{TeV})$} & \multicolumn{5}{|c|}{ Upper limit on $\sigma \mathcal{B A}(\mathrm{fb})$} \\
\hline & $M_{\mathrm{D}}=2 \mathrm{TeV}$ & $M_{\mathrm{D}}=3 \mathrm{TeV}$ & $M_{\mathrm{D}}=4 \mathrm{TeV}$ & $M_{\mathrm{D}}=5 \mathrm{TeV}$ & $M_{\mathrm{D}}=6 \mathrm{TeV}$ \\
\hline 2 & 40 & & & & \\
\hline 2.1 & 30 & & & & \\
\hline 2.2 & 25 & & & & \\
\hline 2.3 & 22 & & & & \\
\hline 2.4 & 15 & & & & \\
\hline 2.5 & 11 & & & & \\
\hline 2.6 & 9.1 & & & & \\
\hline 2.7 & 7.8 & & & & \\
\hline 2.8 & 7.1 & & & & \\
\hline 2.9 & 7.3 & & & & \\
\hline 3 & 7.0 & 7.0 & & & \\
\hline 3.1 & 6.0 & 6.0 & & & \\
\hline 3.2 & 5.1 & 5.1 & & & \\
\hline 3.3 & 4.5 & 4.5 & & & \\
\hline 3.4 & 4.1 & 4.1 & & & \\
\hline 3.5 & 3.3 & 3.3 & & & \\
\hline 3.6 & 2.5 & 2.5 & & & \\
\hline 3.7 & 1.6 & 1.6 & & & \\
\hline 3.8 & 1.1 & 1.1 & & & \\
\hline 3.9 & 0.76 & 0.76 & & & \\
\hline 4 & 0.58 & 0.58 & 0.58 & & \\
\hline 4.1 & 0.46 & 0.46 & 0.46 & & \\
\hline 4.2 & 0.43 & 0.43 & 0.43 & & \\
\hline 4.3 & 0.37 & 0.37 & 0.37 & & \\
\hline 4.4 & 0.35 & 0.35 & 0.35 & & \\
\hline 4.5 & 0.33 & 0.33 & 0.33 & & \\
\hline 4.6 & 0.32 & 0.32 & 0.32 & & \\
\hline 4.7 & 0.30 & 0.30 & 0.30 & & \\
\hline 4.8 & 0.30 & 0.30 & 0.30 & & \\
\hline 4.9 & 0.29 & 0.29 & 0.29 & & \\
\hline 5 & 0.28 & 0.28 & 0.28 & 0.28 & \\
\hline 5.1 & 0.28 & 0.28 & 0.28 & 0.28 & \\
\hline 5.2 & 0.26 & 0.26 & 0.26 & 0.26 & \\
\hline 5.3 & 0.25 & 0.25 & 0.25 & 0.25 & \\
\hline 5.4 & 0.23 & 0.23 & 0.23 & 0.23 & \\
\hline 5.5 & 0.22 & 0.22 & 0.22 & 0.22 & \\
\hline 5.6 & 0.21 & 0.21 & 0.21 & 0.21 & \\
\hline 5.7 & 0.21 & 0.21 & 0.21 & 0.21 & \\
\hline 5.8 & 0.20 & 0.20 & 0.20 & 0.20 & \\
\hline 5.9 & 0.20 & 0.20 & 0.20 & 0.20 & \\
\hline 6. & 0.20 & 0.20 & 0.20 & 0.20 & 0.20 \\
\hline 6.1 & 0.21 & 0.21 & 0.21 & 0.21 & 0.21 \\
\hline 6.2 & 0.22 & 0.22 & 0.22 & 0.22 & 0.22 \\
\hline 6.3 & 0.23 & 0.23 & 0.23 & 0.23 & 0.23 \\
\hline 6.4 & 0.27 & 0.27 & 0.27 & 0.27 & 0.27 \\
\hline 6.5 & 0.31 & 0.31 & 0.31 & 0.31 & 0.31 \\
\hline
\end{tabular}




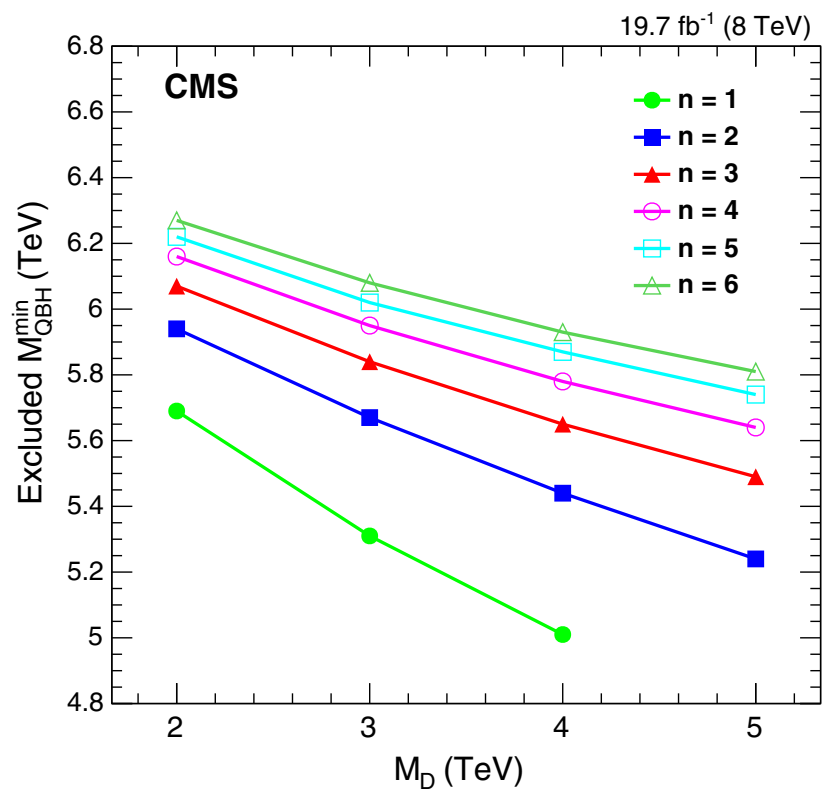

FIG. 12 (color online). Observed 95\% C.L. lower limits on $M_{\mathrm{QBH}}^{\min }$ as a function of the Planck scale $M_{\mathrm{D}}$ and the number of extra dimensions $n$.

The signal shape is almost independent of the number of extra dimensions $n$ and the fundamental Planck scale $M_{\mathrm{D}}$. The $n=1$ case corresponds to RS black holes [27]. In this scenario, $M_{\mathrm{D}}$ is the product of the Planck scale and the exponential factor coming from the warping of the extra dimension.

The 95\% C.L. observed upper limits on $\sigma \mathcal{B A}$ for QBHs are shown in Fig. 11 and reported in Table V. It is commonly assumed [59-61] that $M_{\mathrm{QBH}}^{\min }$ must be greater than or equal to $M_{\mathrm{D}}$. Therefore the cross section limits are presented only for $M_{\mathrm{QBH}}^{\min } \geq M_{\mathrm{D}}$, for different values of $M_{\mathrm{D}}$. The corresponding lower limits on $M_{\mathrm{QBH}}^{\mathrm{min}}$ range from 5.0 to 6.3 TeV, depending on the model parameters, and are shown in Fig. 12 and Table VI as a function of $M_{\mathrm{D}}$ and $n$. These limits extend those obtained in Refs. [3,4], where the same benchmark models were considered in the context of a multijet search.

TABLE VI. Observed $95 \%$ C.L. lower limits on $M_{\mathrm{QBH}}^{\min }$ for different numbers of extra dimensions $n$ and several values of $M_{\mathrm{D}}$.

\begin{tabular}{lcccc}
\hline \hline & \multicolumn{4}{c}{$M_{\mathrm{D}}(\mathrm{TeV})$} \\
\cline { 2 - 5 }$n$ & 2 & 3 & 4 & 5 \\
\hline 1 & 5.7 & 5.3 & 5.0 & \\
2 & 5.9 & 5.7 & 5.4 & 5.2 \\
3 & 6.1 & 5.8 & 5.7 & 5.5 \\
4 & 6.2 & 6.0 & 5.8 & 5.6 \\
5 & 6.2 & 6.0 & 5.9 & 5.7 \\
6 & 6.3 & 6.0 & 5.9 & 5.8 \\
\hline \hline
\end{tabular}

\section{SUMMARY}

A search for resonances and quantum black holes has been performed in inclusive and $b$-tagged dijet mass spectra measured with the CMS detector at the LHC. The data set corresponds to $19.7 \mathrm{fb}^{-1}$ of integrated luminosity collected in proton-proton collisions at $\sqrt{s}=8 \mathrm{TeV}$. The inclusive search has been conducted for narrow resonances and, for the first time, for wide resonances with relative widths up to $30 \%$ of the resonance mass. No evidence for new particle production is found. Upper limits at 95\% C.L. on the product of the cross section, branching fraction into dijets, and acceptance are provided for all generic searches. Specific lower limits are set on the masses of string resonances, excited quarks, axigluons, colorons, color-octet scalar resonances, scalar diquarks, $W^{\prime}$ and $Z^{\prime}$ bosons, and RS gravitons. For the first time, an exclusion limit is set for excited $b$ quarks. The lower mass limits reach up to $5 \mathrm{TeV}$, depending on the model, and extend previous exclusions based on the dijet mass search technique. Quantum black holes up to a mass ranging from 5.0 to $6.3 \mathrm{TeV}$ are also excluded at $95 \%$ C.L., depending on the model.

\section{ACKNOWLEDGMENTS}

We congratulate our colleagues in the CERN accelerator departments for the excellent performance of the LHC and thank the technical and administrative staffs at CERN and at other CMS institutes for their contributions to the success of the CMS effort. In addition, we gratefully acknowledge the computing centers and personnel of the Worldwide LHC Computing Grid for delivering so effectively the computing infrastructure essential to our analyses. Finally, we acknowledge the enduring support for the construction and operation of the LHC and the CMS detector provided by the following funding agencies: the Austrian Federal Ministry of Science, Research and Economy and the Austrian Science Fund; the Belgian Fonds de la Recherche Scientifique, and Fonds voor Wetenschappelijk Onderzoek; the Brazilian Funding Agencies (CNPq, CAPES, FAPERJ, and FAPESP); the Bulgarian Ministry of Education and Science; CERN; the Chinese Academy of Sciences, Ministry of Science and Technology, and National Natural Science Foundation of China; the Colombian Funding Agency (COLCIENCIAS); the Croatian Ministry of Science, Education and Sport, and the Croatian Science Foundation; the Research Promotion Foundation, Cyprus; the Ministry of Education and Research, Estonian Research Council via IUT23-4 and IUT23-6 and European Regional Development Fund, Estonia; the Academy of Finland, Finnish Ministry of Education and Culture, and Helsinki Institute of Physics; the Institut National de Physique Nucléaire et de Physique des Particules / CNRS, and Commissariat à l'Énergie Atomique et aux Énergies Alternatives / CEA, France; the Bundesministerium für Bildung und Forschung, 
Deutsche Forschungsgemeinschaft, and HelmholtzGemeinschaft Deutscher Forschungszentren, Germany; the General Secretariat for Research and Technology, Greece; the National Scientific Research Foundation, and National Innovation Office, Hungary; the Department of Atomic Energy and the Department of Science and Technology, India; the Institute for Studies in Theoretical Physics and Mathematics, Iran; the Science Foundation, Ireland; the Istituto Nazionale di Fisica Nucleare, Italy; the Ministry of Science, ICT and Future Planning, and National Research Foundation (NRF), Republic of Korea; the Lithuanian Academy of Sciences; the Ministry of Education, and University of Malaya (Malaysia); the Mexican Funding Agencies (CINVESTAV, CONACYT, SEP, and UASLP-FAI); the Ministry of Business, Innovation and Employment, New Zealand; the Pakistan Atomic Energy Commission; the Ministry of Science and Higher Education and the National Science Centre, Poland; the Fundação para a Ciência e a Tecnologia, Portugal; JINR, Dubna; the Ministry of Education and Science of the Russian Federation, the Federal Agency of Atomic Energy of the Russian Federation, Russian Academy of Sciences, and the Russian Foundation for Basic Research; the Ministry of Education, Science and Technological Development of Serbia; the Secretaría de Estado de Investigación, Desarrollo e Innovación and Programa ConsoliderIngenio 2010, Spain; the Swiss Funding Agencies (ETH Board, ETH Zurich, PSI, SNF, UniZH, Canton Zurich, and SER); the Ministry of Science and Technology, Taipei; the Thailand Center of Excellence in Physics, the Institute for the Promotion of Teaching Science and Technology of Thailand, Special Task Force for Activating Research and the National Science and Technology Development Agency of Thailand; the Scientific and Technical Research Council of Turkey, and Turkish Atomic Energy Authority; the National Academy of Sciences of Ukraine, and State Fund for Fundamental Researches, Ukraine; the Science and Technology Facilities Council, UK; the U.S. Department of Energy, and the US National Science Foundation. Individuals have received support from the Marie-Curie program and the European Research Council and EPLANET (European Union); the Leventis Foundation; the A. P. Sloan Foundation; the Alexander von Humboldt Foundation; the Belgian Federal Science Policy Office; the Fonds pour la Formation à la Recherche dans l'Industrie et dans l'Agriculture (FRIA-Belgium); the Agentschap voor Innovatie door Wetenschap en Technologie (IWT-Belgium); the Ministry of Education, Youth and Sports (MEYS) of the Czech Republic; the Council of Science and Industrial Research, India; the HOMING PLUS program of Foundation for Polish Science, cofinanced from European Union, Regional Development Fund; the Compagnia di San Paolo (Torino); the Consorzio per la Fisica (Trieste); MIUR project 20108T4XTM (Italy); the Thalis and Aristeia programs cofinanced by EU-ESF and the Greek NSRF; and the National Priorities Research Program by Qatar National Research Fund.

\section{APPENDIX: CROSS SECTION CALCULATION FOR WIDE RESONANCES}

Cross sections for narrow resonances are often given in the narrow width approximation, where the subprocess cross section

$$
\hat{\sigma}(\hat{s}) \propto \delta\left(\hat{s}-M_{X}^{2}\right)
$$

is integrated over the PDFs (Sec. 2.2.11 in Ref. [12]). Here $\hat{s}=m^{2}$ is the square of the diparton mass, $M_{X}$ is the resonance mass, and the delta function implies that the PDFs are evaluated at only those values of fractional momenta $x_{1}$ and $x_{2}$ that correspond to the resonance pole: $M_{X}^{2}=\hat{s}=x_{1} x_{2} s$, where $s$ is the square of the protonproton collision energy. Cross sections calculated in the narrow-width approximation are appropriate for comparison to CMS upper limits for narrow resonances, because the dijet mass resonance shapes used in that search correspond to a relative resonance width $(\Gamma / M)$ much smaller than the detector resolution.

Multiple processes can contribute to the total cross section for wide resonance production. The $s$-channel process, the annihilation of two initial state partons into the resonance, is usually the most significant contribution and is the process searched for by this analysis. The $s$-channel cross section is evaluated by replacing the delta function in Eq. (A1) with a full relativistic Breit-Wigner resonance shape, before integrating over the PDFs. The $t$-channel process, where the new particle is exchanged between the incoming partons, often has an appreciable contribution to the cross section but it does not peak sharply in diparton mass and may be absorbed into the background shape during a search. The interference process, including interference between the multijet background processes and both the $s$ - and $t$-channel signal processes, could often significantly modify the resonance shape far off the resonance pole. Interference contributions depend on the type of resonance considered and are not included in the resonance shape used in our search. Our calculation of the wide resonance cross section is an approximation that considers only the $s$-channel term, to which limits from our search should be compared.

The cross section calculations for wide resonances employ a resonance shape for the $s$-channel resonances as a function of $\hat{s}-M_{X}^{2}$. In order for this calculated cross section to be comparable to the resonance upper limits, we have used the same shape for the underlying parton-parton scattering subprocess cross section as is used in the search to set limits. The shape corresponds to an RS graviton resonance. The generator used, PYTHIA6, models that shape 
with the following, general Breit-Wigner resonance formula [Eq. (7.47) in [62]]:

$$
\hat{\sigma}_{i \rightarrow R \rightarrow f}(\hat{s}) \propto \frac{\pi}{\hat{s}} \frac{H_{R}^{(i)}(\hat{s}) H_{R}^{(f)}(\hat{s})}{\left(\hat{s}-M_{X}^{2}\right)^{2}+H_{R}^{2}(\hat{s})},
$$

where

$$
H_{R}(\hat{s})=\frac{\hat{s} \Gamma_{R}}{M_{X}}
$$

and $\Gamma_{R}$ is the full resonance width. For the $\mathrm{RS}$ graviton resonance

$$
H_{R}^{(i, f)}(\hat{s})=\left(\frac{\hat{s}}{M_{X}^{2}}\right) \frac{\hat{s} \Gamma_{R}^{(i, f)}}{M_{X}}
$$

where $\Gamma_{R}^{(i, f)}$ are the partial widths for the initial state $i$ and final state $f$. We note that the term $\hat{s} / M_{X}^{2}$ in Eq. (A4) significantly affects the resonance shape far away from the resonance pole, suppressing the tail at low diparton mass. This term is appropriate for resonances that have a width proportional to the cube of the resonance mass, like the RS graviton or the color-octet scalar. Even with this suppression, the enhancement at low dijet mass due to convolution of the tail with PDFs is visible in Fig. 7 for resonances with the highest widths and masses.

TABLE VII. Correction factors defined as the ratio of the full cross section obtained from Eqs. (A2)-(A4) to the cross section from the

\begin{tabular}{|c|c|c|c|c|c|c|c|c|}
\hline \multirow[b]{2}{*}{ Mass (TeV) } & \multicolumn{8}{|c|}{ Fractional width $(\Gamma / \mathrm{M})$} \\
\hline & $10^{-5}$ & 0.015 & 0.05 & 0.1 & 0.15 & 0.2 & 0.25 & 0.3 \\
\hline \multicolumn{9}{|c|}{ Correction factors for $q \bar{q}$ resonances } \\
\hline 1.25 & 1.00 & 1.00 & 0.97 & 0.85 & 0.81 & 0.79 & 0.77 & 0.76 \\
\hline 1.50 & 1.00 & 1.00 & 0.97 & 0.85 & 0.81 & 0.79 & 0.77 & 0.77 \\
\hline 1.75 & 1.00 & 1.00 & 0.97 & 0.85 & 0.82 & 0.79 & 0.78 & 0.78 \\
\hline 2.00 & 1.00 & 1.01 & 0.98 & 0.86 & 0.83 & 0.81 & 0.80 & 0.80 \\
\hline 2.25 & 1.00 & 1.01 & 0.98 & 0.87 & 0.84 & 0.83 & 0.83 & 0.84 \\
\hline 2.50 & 1.00 & 1.01 & 0.99 & 0.89 & 0.87 & 0.87 & 0.88 & 0.89 \\
\hline 2.75 & 1.00 & 1.01 & 1.01 & 0.92 & 0.91 & 0.92 & 0.94 & 0.97 \\
\hline 3.00 & 1.00 & 1.01 & 1.03 & 0.96 & 0.97 & 1.00 & 1.04 & 1.09 \\
\hline 3.25 & 1.00 & 1.02 & 1.05 & 1.02 & 1.06 & 1.11 & 1.18 & 1.26 \\
\hline 3.50 & 1.00 & 1.02 & 1.09 & 1.12 & 1.20 & 1.29 & 1.40 & 1.52 \\
\hline 3.75 & 1.00 & 1.03 & 1.14 & 1.26 & 1.41 & 1.57 & 1.74 & 1.92 \\
\hline 4.00 & 1.00 & 1.04 & 1.22 & 1.50 & 1.75 & 2.02 & 2.30 & 2.60 \\
\hline 4.25 & 1.00 & 1.05 & 1.34 & 1.91 & 2.35 & 2.81 & 3.29 & 3.80 \\
\hline 4.50 & 1.00 & 1.06 & 1.54 & 2.67 & 3.46 & 4.27 & 5.12 & 6.03 \\
\hline 4.75 & 1.00 & 1.08 & 1.87 & 4.16 & 5.66 & 7.20 & 8.82 & 10.5 \\
\hline 5.00 & 1.00 & 1.11 & 2.45 & 7.36 & 10.4 & 13.5 & 16.9 & 20.4 \\
\hline 5.25 & 1.00 & 1.14 & 3.52 & 14.8 & 21.4 & 28.4 & 35.7 & 43.6 \\
\hline 5.50 & 1.00 & 1.19 & 5.60 & 33.3 & 49.1 & 65.6 & 83.2 & 102 \\
\hline \multicolumn{9}{|c|}{ Correction factors for $g g$ resonances } \\
\hline 1.25 & 1.00 & 1.01 & 0.98 & 0.88 & 0.85 & 0.84 & 0.84 & 0.85 \\
\hline 1.50 & 1.00 & 1.01 & 0.99 & 0.89 & 0.87 & 0.87 & 0.88 & 0.90 \\
\hline 1.75 & 1.00 & 1.01 & 1.00 & 0.92 & 0.91 & 0.92 & 0.94 & 0.96 \\
\hline 2.00 & 1.00 & 1.01 & 1.01 & 0.95 & 0.95 & 0.98 & 1.01 & 1.06 \\
\hline 2.25 & 1.00 & 1.01 & 1.03 & 0.99 & 1.02 & 1.06 & 1.12 & 1.18 \\
\hline 2.50 & 1.00 & 1.02 & 1.05 & 1.06 & 1.11 & 1.18 & 1.27 & 1.37 \\
\hline 2.75 & 1.00 & 1.02 & 1.08 & 1.15 & 1.24 & 1.36 & 1.49 & 1.63 \\
\hline 3.00 & 1.00 & 1.02 & 1.11 & 1.28 & 1.44 & 1.62 & 1.81 & 2.03 \\
\hline 3.25 & 1.00 & 1.03 & 1.16 & 1.49 & 1.74 & 2.02 & 2.31 & 2.64 \\
\hline 3.50 & 1.00 & 1.03 & 1.23 & 1.81 & 2.22 & 2.65 & 3.11 & 3.61 \\
\hline 3.75 & 1.00 & 1.04 & 1.33 & 2.34 & 3.00 & 3.70 & 4.44 & 5.23 \\
\hline 4.00 & 1.00 & 1.05 & 1.46 & 3.26 & 4.36 & 5.52 & 6.75 & 8.07 \\
\hline 4.25 & 1.00 & 1.06 & 1.67 & 4.94 & 6.86 & 8.87 & 11.01 & 13.3 \\
\hline 4.50 & 1.00 & 1.08 & 1.99 & 8.20 & 11.7 & 15.4 & 19.4 & 23.6 \\
\hline 4.75 & 1.00 & 1.10 & 2.51 & 15.0 & 21.9 & 29.1 & 36.9 & 45.3 \\
\hline 5.00 & 1.00 & 1.12 & 3.43 & 30.4 & 44.9 & 60.2 & 76.6 & 94.5 \\
\hline 5.25 & 1.00 & 1.16 & 5.17 & 68.3 & 102 & 137 & 175 & 216 \\
\hline 5.50 & 1.00 & 1.22 & 8.73 & 172 & 257 & 348 & 445 & 552 \\
\hline
\end{tabular}
narrow-width approximation calculations, as a function of the resonance mass, for $q \bar{q}$ and $g g$ resonances and for eight different resonance widths in proton-proton collisions at $\sqrt{s}=8 \mathrm{TeV}$. 
We calculate the full wide resonance cross section from $s$-channel production by integrating the Breit-Wigner resonance shape defined by Eqs. (A2)-(A4), over the PDFs. Table VII shows the full cross section divided by the cross section in the narrow-width approximation as a function of the resonance mass and width, for both $q \bar{q}$ and $g g$ resonances. This ratio is close to unity for narrow resonances, for which the full cross section and the narrowwidth approximation cross section are naturally the same. For wide resonances at high resonance mass this ratio can be significantly greater than 1 , because the convolution of the PDFs with the low mass tail of the Breit-Wigner result in a large cross section at low diparton mass. For wide resonances the values reported in Table VII can be applied as a multiplicative correction to the narrow-width approximation cross sections to get an appropriate resonance cross section to compare with our experimental upper limits on cross section. This is done in Fig. 9 to obtain the model cross section presented and to set mass limits for axigluons/ colorons and color-octet scalars. The correction factor for axigluons, which are $q \bar{q}$ resonances of width $\Gamma_{R}=\alpha_{S} M_{X}$, is 1.1 at a mass of $3.5 \mathrm{TeV}$. The correction factor for coloroctet scalars, which are $g g$ resonances of width $\Gamma_{R}=5 \alpha_{S} M_{X} / 6$, is 1.1 at a mass of $2.5 \mathrm{TeV}$. So for these resonances, at mass values close to our mass limit, the full cross section is close to the cross section calculated in the narrow-width approximation. We recommend the same procedure, using Table VII, for users of our limits on the wide-resonance cross section, as this will ensure that the resonance shape used to calculate the cross section matches the resonance shape we used to set limits.

For resonances with widths that are directly proportional to the resonance mass, like axigluons or colorons, the following term is normally used instead of Eq. (A4) to describe the resonance line shape:

$$
H_{R}^{(i, f)}(\hat{s})=\frac{\hat{s} \Gamma_{R}^{(i, f)}}{M_{X}}
$$

For many wide resonances of interest this term produces a resonance shape with a very large tail at low mass: a cross section that falls rapidly with increasing diparton mass, like the multijet background. This shape at low dijet mass would be largely absorbed into the multijet background definition of our search. Like the multijet background, the full cross section for this wide shape is mainly determined by the lowest diparton mass considered. This shape is therefore difficult to use in a well-defined fashion in a search that sets upper limits on a resonance cross section, because the cross section is only weakly dependent on the resonance pole mass. Thus, we have limited the wide resonance search to the shape defined by Eq. (A4). Our wide resonance search results are still applicable for a range of resonance widths and masses even for resonances that have a shape defined by Eq. (A5). As long as the full cross section for the true resonance line shape integrated over the mass interval of the CMS search is not larger than about 20 times the narrow-width approximation cross section, the results of the CMS search are approximately valid and applicable. This approximate range of validity is derived by comparing Table IV with VII. The boundary of validity of the limits shown in Table IV has an average ratio value of about 20 in Table VII. Note that our limits are valid if the condition reported in Sec. VI holds so that the low mass tail does not significantly affect the shape analysis. Thus, to first approximation, only this comparison of the full resonance cross section to the narrow width cross section matters in determining validity of our limits, independent of the shape of the low mass tail.
[1] CMS Collaboration, Phys. Rev. Lett. 105, 211801 (2010).

[2] S. Chatrchyan et al. (CMS Collaboration), Phys. Lett. B 704, 123 (2011).

[3] S. Chatrchyan et al. (CMS Collaboration), J. High Energy Phys. 04 (2012) 061.

[4] CMS Collaboration, J. High Energy Phys. 07 (2013) 178.

[5] CMS Collaboration, J. High Energy Phys. 01 (2013) 013.

[6] CMS Collaboration, Phys. Rev. D 87, 114015 (2013).

[7] ATLAS Collaboration, Phys. Rev. Lett. 105, 161801 (2010).

[8] ATLAS Collaboration, New J. Phys. 13, 053044 (2011).

[9] G. Aad et al. (ATLAS Collaboration), Phys. Lett. B 708, 37 (2012).

[10] ATLAS Collaboration, J. High Energy Phys. 01 (2013) 029.
[11] ATLAS Collaboration, Search for new phenomena in the dijet mass distribution using pp collision data at $\sqrt{s}=$ $8 \mathrm{TeV}$ with the ATLAS detector, arXiv:1407.1376.

[12] R. M. Harris and K. Kousouris, Int. J. Mod. Phys. A 26, 5005 (2011).

[13] L. A. Anchordoqui, H. Goldberg, D. Lust, S. Nawata, S. Stieberger, and T. R. Taylor, Phys. Rev. Lett. 101, 241803 (2008).

[14] S. Cullen, M. Perelstein, and M. E. Peskin, Phys. Rev. D 62 , 055012 (2000).

[15] J. L. Hewett and T. G. Rizzo, Phys. Rep. 183, 193 (1989).

[16] U. Baur, I. Hinchliffe, and D. Zeppenfeld, Int. J. Mod. Phys. A 02, 1285 (1987).

[17] U. Baur, M. Spira, and P. M. Zerwas, Phys. Rev. D 42, 815 (1990). 
[18] P. H. Frampton and S. L. Glashow, Phys. Lett. B 190, 157 (1987).

[19] J. Bagger, C. Schmidt, and S. King, Phys. Rev. D 37, 1188 (1988).

[20] R. S. Chivukula, A. Farzinnia, E. H. Simmons, and R. Foadi, Phys. Rev. D 85, 054005 (2012).

[21] E. H. Simmons, Phys. Rev. D 55, 1678 (1997).

[22] T. Han, I. Lewis, and Z. Liu, J. High Energy Phys. 12 (2010) 085.

[23] E. Eichten, I. Hinchliffe, K. D. Lane, and C. Quigg, Rev. Mod. Phys. 56, 579 (1984).

[24] L. Randall and R. Sundrum, Phys. Rev. Lett. 83, 3370 (1999).

[25] L. Randall and R. Sundrum, Phys. Rev. Lett. 83, 4690 (1999).

[26] J. Bijnens, P. Eerola, M. Maul, A. Mansson, and T. Sjöstrand, Phys. Lett. B 503, 341 (2001).

[27] P. Meade and L. Randall, J. High Energy Phys. 05 (2008) 003.

[28] X. Calmet, W. Gong, and S. D. H. Hsu, Phys. Lett. B 668, 20 (2008).

[29] D. M. Gingrich, J. Phys. G 37, 105008 (2010).

[30] N. Arkani-Hamed, S. Dimopoulos, and G. Dvali, Phys. Lett. B 429, 263 (1998).

[31] N. Arkani-Hamed, S. Dimopoulos, and G. Dvali, Phys. Rev. D 59, 086004 (1999).

[32] CMS Collaboration, JINST 3, S08004 (2008).

[33] CMS Collaboration, CMS Physics Analysis Summary Report No. CMS-PAS-PFT-09-001, 2009, http://cdsweb .cern.ch/record/1194487.

[34] CMS Collaboration, CMS Physics Analysis Summary Report No. CMS-PAS-PFT-10-001, 2010, http://cdsweb .cern.ch/record/1247373.

[35] M. Cacciari, G. P. Salam, and G. Soyez, J. High Energy Phys. 04 (2008) 063.

[36] M. Cacciari and G. P. Salam, Phys. Lett. B 641, 57 (2006).

[37] M. Cacciari, G. P. Salam, and G. Soyez, Eur. Phys. J. C 72, 1896 (2012).

[38] M. Cacciari and G. P. Salam, Phys. Lett. B 659, 119 (2008).

[39] CMS Collaboration, JINST 6, P11002 (2011).

[40] CMS Collaboration, CMS Physics Analysis Summary Report No. CMS-PAS-JME-10-003, 2010, http://cdsweb .cern.ch/record/1279362.

[41] M. Cacciari, J. Rojo, G. P. Salam, and G. Soyez, J. High Energy Phys. 12 (2008) 032.
[42] D. Krohn, J. Thaler, and L.-T. Wang, J. High Energy Phys. 02 (2010) 084.

[43] A. Abdesselam et al., Eur. Phys. J. C 71, 1661 (2011).

[44] CMS Collaboration, JINST 8, P04013 (2013).

[45] T. Sjöstrand, P. Edén, C. Friberg, L. Lönnblad, G. Miu, S. Mrenna, and E. Norrbin, Comput. Phys. Commun. 135, 238 (2001).

[46] R. Field, Early LHC underlying event data-findings and surprises, arXiv:1010.3558.

[47] S. Agostinelli et al. (GEANT4 Collaboration), Nucl. Instrum. Methods Phys. Res., Sect. A 506, 250 (2003).

[48] J. Pumplin, D. R. Stump, J. Huston, H. L. Lai, P. M. Nadolsky, and W.-K. Tung, J. High Energy Phys. 07 (2002) 012.

[49] CMS Collaboration, Phys. Rev. Lett. 106, 029902 (2011).

[50] T. Aaltonen et al. (CDF Collaboration), Phys. Rev. D 79, 112002 (2009).

[51] R. G. Lomax and D. L. Hahs-Vaughn, Statistical Concepts: A Second Course (Routledge Academic, London, 2007).

[52] T. Sjöstrand, S. Mrenna, and P. Z. Skands, Comput. Phys. Commun. 178, 852 (2008).

[53] R. Corke and T. Sjöstrand, J. High Energy Phys. 03 (2011) 032.

[54] CMS Collaboration, CMS Physics Analysis Summary Report No. CMS-PAS-LUM-13-001, 2013, http://cdsweb .cern.ch/record/1598864.

[55] K. Nakamura et al. (Particle Data Group), J. Phys. G 37, 075021 (2010), see Chap. 33, Statistics, by G. Cowan.

[56] A. Caldwell, D. Kollar, and K. Kroninger, Comput. Phys. Commun. 180, 2197 (2009).

[57] S. Abdullin, P. Azzi, F. Beaudette, P. Janot, and A. Perrotta (CMS Collaboration), J. Phys. Conf. Ser. 331, 032049 (2011).

[58] D. M. Gingrich, Comput. Phys. Commun. 181, 1917 (2010).

[59] T. Banks and W. Fischler, arXiv:hep-th/9906038.

[60] S. Dimopoulos and G. L. Landsberg, Phys. Rev. Lett. 87, 161602 (2001).

[61] S. B. Giddings and S. D. Thomas, Phys. Rev. D 65, 056010 (2002).

[62] T. Sjöstrand, S. Mrenna, and P. Z. Skands, J. High Energy Phys. 05 (2006) 026.

V. Khachatryan, ${ }^{1}$ A. M. Sirunyan, ${ }^{1}$ A. Tumasyan, ${ }^{1}$ W. Adam, ${ }^{2}$ T. Bergauer, ${ }^{2}$ M. Dragicevic, ${ }^{2}$ J. Erö, ${ }^{2}$ M. Friedl, ${ }^{2}$ R. Frühwirth, ${ }^{2, b}$ V. M. Ghete, ${ }^{2}$ C. Hartl, ${ }^{2}$ N. Hörmann, ${ }^{2}$ J. Hrubec, ${ }^{2}$ M. Jeitler, ${ }^{2, b}$ W. Kiesenhofer, ${ }^{2}$ V. Knünz, ${ }^{2}$ M. Krammer, ${ }^{2, b}$ I. Krätschmer, ${ }^{2}$ D. Liko, ${ }^{2}$ I. Mikulec, ${ }^{2}$ D. Rabady, ${ }^{2, \mathrm{c}}$ B. Rahbaran, ${ }^{2}$ H. Rohringer, ${ }^{2}$ R. Schöfbeck, ${ }^{2}$ J. Strauss, ${ }^{2}$ W. Treberer-Treberspurg, ${ }^{2}$ W. Waltenberger, ${ }^{2}$ C.-E. Wulz, ${ }^{2, b}$ V. Mossolov, ${ }^{3}$ N. Shumeiko, ${ }^{3}$ J. Suarez Gonzalez, ${ }^{3}$ S. Alderweireldt, ${ }^{4}$ S. Bansal, ${ }^{4}$ T. Cornelis, ${ }^{4}$ E. A. De Wolf, ${ }^{4}$ X. Janssen, ${ }^{4}$ A. Knutsson, ${ }^{4}$ J. Lauwers, ${ }^{4}$ S. Luyckx,${ }^{4}$ S. Ochesanu, ${ }^{4}$ R. Rougny, ${ }^{4}$ M. Van De Klundert, ${ }^{4}$ H. Van Haevermaet, ${ }^{4}$ P. Van Mechelen, ${ }^{4}$ N. Van Remortel, ${ }^{4}$ A. Van Spilbeeck, ${ }^{4}$ F. Blekman, ${ }^{5}$ S. Blyweert, ${ }^{5}$ J. D'Hondt, ${ }^{5}$ N. Daci, ${ }^{5}$ N. Heracleous, ${ }^{5}$ J. Keaveney, ${ }^{5}$ S. Lowette, ${ }^{5}$ M. Maes, ${ }^{5}$ A. Olbrechts, ${ }^{5}$ Q. Python, ${ }^{5}$ D. Strom, ${ }^{5}$ S. Tavernier, ${ }^{5}$ W. Van Doninck, ${ }^{5}$ P. Van Mulders, ${ }^{5}$ G. P. Van Onsem, ${ }^{5}$ I. Villella, ${ }^{5}$ C. Caillol, ${ }^{6}$ B. Clerbaux, ${ }^{6}$ G. De Lentdecker, ${ }^{6}$ D. Dobur, ${ }^{6}$ L. Favart, ${ }^{6}$ A. P. R. Gay, ${ }^{6}$ A. Grebenyuk, ${ }^{6}$ A. Léonard, ${ }^{6}$ 
A. Mohammadi, ${ }^{6}$ L. Perniè, ${ }^{6, c}$ A. Randle-conde, ${ }^{6}$ T. Reis, ${ }^{6}$ T. Seva, ${ }^{6}$ L. Thomas, ${ }^{6}$ C. Vander Velde, ${ }^{6}$ P. Vanlaer, ${ }^{6}$ J. Wang, ${ }^{6}$ F. Zenoni, ${ }^{6}$ V. Adler, ${ }^{7}$ K. Beernaert, ${ }^{7}$ L. Benucci, ${ }^{7}$ A. Cimmino, ${ }^{7}$ S. Costantini, ${ }^{7}$ S. Crucy, ${ }^{7}$ S. Dildick, ${ }^{7}$ A. Fagot, ${ }^{7}$ G. Garcia, ${ }^{7}$ J. Mccartin, ${ }^{7}$ A. A. Ocampo Rios, ${ }^{7}$ D. Poyraz, ${ }^{7}$ D. Ryckbosch, ${ }^{7}$ S. Salva Diblen, ${ }^{7}$ M. Sigamani, ${ }^{7}$ N. Strobbe, ${ }^{7}$ F. Thyssen, ${ }^{7}$ M. Tytgat, ${ }^{7}$ E. Yazgan ${ }^{7}$ N. Zaganidis, ${ }^{7}$ S. Basegmez,${ }^{8}$ C. Beluffi, ${ }^{8, d}$ G. Bruno, ${ }^{8}$ R. Castello, ${ }^{8}$ A. Caudron, ${ }^{8}$ L. Ceard ${ }^{8}$ G. G. Da Silveira, ${ }^{8}$ C. Delaere, ${ }^{8}$ T. du Pree, ${ }^{8}$ D. Favart, ${ }^{8}$ L. Forthomme, ${ }^{8}$ A. Giammanco, ${ }^{8, e}$ J. Hollar, ${ }^{8}$ A. Jafari, ${ }^{8}$ P. Jez, ${ }^{8}$ M. Komm, ${ }^{8}$ V. Lemaitre, ${ }^{8}$ C. Nuttens,${ }^{8}$ L. Perrini, ${ }^{8}$ A. Pin, ${ }^{8}$ K. Piotrzkowski, ${ }^{8}$ A. Popov, ${ }^{8, f}$ L. Quertenmont, ${ }^{8}$ M. Selvaggi, ${ }^{8}$ M. Vidal Marono, ${ }^{8}$ J. M. Vizan Garcia, ${ }^{8}$ N. Beliy, ${ }^{9}$ T. Caebergs, ${ }^{9}$ E. Daubie, ${ }^{9}$ G. H. Hammad, ${ }^{9}$ W. L. Aldá Júnior, ${ }^{10}$ G. A. Alves, ${ }^{10}$ L. Brito, ${ }^{10}$ M. Correa Martins Junior, ${ }^{10}$ T. Dos Reis Martins, ${ }^{10}$ J. Molina, ${ }^{10}$ C. Mora Herrera, ${ }^{10}$ M. E. Pol, ${ }^{10}$ P. Rebello Teles,${ }^{10}$ W. Carvalho, ${ }^{11}$ J. Chinellato, ${ }^{11, g}$ A. Custódio, ${ }^{11}$ E. M. Da Costa, ${ }^{11}$ D. De Jesus Damiao, ${ }^{11}$ C. De Oliveira Martins, ${ }^{11}$ S. Fonseca De Souza, ${ }^{11}$ H. Malbouisson, ${ }^{11}$ D. Matos Figueiredo, ${ }^{11}$ L. Mundim, ${ }^{11}$ H. Nogima, ${ }^{11}$ W. L. Prado Da Silva, ${ }^{11}$ J. Santaolalla, ${ }^{11}$ A. Santoro, ${ }^{11}$ A. Sznajder, ${ }^{11}$

E. J. Tonelli Manganote, ${ }^{1, g}$ A. Vilela Pereira, ${ }^{11}$ C. A. Bernardes, ${ }^{12 b}$ S. Dogra ${ }^{12 a}$ T. R. Fernandez Perez Tomei, ${ }^{12 a}$ E. M. Gregores, ${ }^{12 b}$ P. G. Mercadante, ${ }^{12 b}$ S. F. Novaes, ${ }^{12 a}$ Sandra S. Padula, ${ }^{12 a}$ A. Aleksandrov, ${ }^{13}$ V. Genchev,,${ }^{13, c}$ R. Hadjiiska, ${ }^{13}$ P. Iaydjiev, ${ }^{13}$ A. Marinov, ${ }^{13}$ S. Piperov, ${ }^{13}$ M. Rodozov, ${ }^{13}$ S. Stoykova, ${ }^{13}$ G. Sultanov,${ }^{13}$ M. Vutova, ${ }^{13}$ A. Dimitrov, ${ }^{14}$ I. Glushkov, ${ }^{14}$ L. Litov, ${ }^{14}$ B. Pavlov, ${ }^{14}$ P. Petkov, ${ }^{14}$ J. G. Bian, ${ }^{15}$ G. M. Chen, ${ }^{15}$ H. S. Chen, ${ }^{15}$ M. Chen, ${ }^{15}$ T. Cheng, ${ }^{15}$ R. Du, ${ }^{15}$ C. H. Jiang, ${ }^{15}$ R. Plestina, ${ }^{15, \mathrm{~h}}$ F. Romeo, ${ }^{15}$ J. Tao, ${ }^{15}$ Z. Wang,,${ }^{15}$ C. Asawatangtrakuldee,${ }^{16}$ Y. Ban, ${ }^{16}$ S. Liu, ${ }^{16}$ Y. Mao, ${ }^{16}$ S. J. Qian, ${ }^{16}$ D. Wang, ${ }^{16}$ Z. Xu, ${ }^{16}$ L. Zhang,${ }^{16}$ W. Zou, ${ }^{16}$ C. Avila, ${ }^{17}$ A. Cabrera, ${ }^{17}$ L. F. Chaparro Sierra, ${ }^{17}$ C. Florez, ${ }^{17}$ J. P. Gomez,${ }^{17}$ B. Gomez Moreno, ${ }^{17}$ J. C. Sanabria,${ }^{17}$ N. Godinovic, ${ }^{18}$ D. Lelas, ${ }^{18}$ D. Polic, ${ }^{18}$ I. Puljak, ${ }^{18}$ Z. Antunovic, ${ }^{19}$ M. Kovac, ${ }^{19}$ V. Brigljevic, ${ }^{20}$ K. Kadija,${ }^{20}$ J. Luetic,${ }^{20}$ D. Mekterovic,${ }^{20}$ L. Sudic, ${ }^{20}$ A. Attikis, ${ }^{21}$ G. Mavromanolakis, ${ }^{21}$ J. Mousa, ${ }^{21}$ C. Nicolaou, ${ }^{21}$ F. Ptochos, ${ }^{21}$ P. A. Razis, ${ }^{21}$ H. Rykaczewski, ${ }^{21}$ M. Bodlak, ${ }^{22}$ M. Finger, ${ }^{22}$ M. Finger Jr., ${ }^{22, i}$ Y. Assran, ${ }^{23, j}$ S. Elgammal, ${ }^{23, \mathrm{k}}$ A. Ellithi Kamel, ${ }^{23,1}$ A. Radi, ${ }^{23, m, n}$ M. Kadastik, ${ }^{24}$ M. Murumaa, ${ }^{24}$ M. Raidal, ${ }^{24}$ A. Tiko, ${ }^{24}$ P. Eerola, ${ }^{25}$ M. Voutilainen, ${ }^{25}$ J. Härkönen, ${ }^{26}$ V. Karimäki, ${ }^{26}$ R. Kinnunen, ${ }^{26}$ M. J. Kortelainen, ${ }^{26}$

T. Lampén, ${ }^{26}$ K. Lassila-Perini, ${ }^{26}$ S. Lehti, ${ }^{26}$ T. Lindén, ${ }^{26}$ P. Luukka, ${ }^{26}$ T. Mäenpääa, ${ }^{26}$ T. Peltola, ${ }^{26}$ E. Tuominen, ${ }^{26}$ J. Tuominiemi, ${ }^{26}$ E. Tuovinen, ${ }^{26}$ L. Wendland ${ }^{26}$ J. Talvitie, ${ }^{27}$ T. Tuuva, ${ }^{27}$ M. Besancon, ${ }^{28}$ F. Couderc, ${ }^{28}$ M. Dejardin, ${ }^{28}$ D. Denegri, ${ }^{28}$ B. Fabbro, ${ }^{28}$ J. L. Faure, ${ }^{28}$ C. Favaro, ${ }^{28}$ F. Ferri, ${ }^{28}$ S. Ganjour, ${ }^{28}$ A. Givernaud ${ }^{28}$ P. Gras, ${ }^{28}$ G. Hamel de Monchenault, ${ }^{28}$ P. Jarry, ${ }^{28}$ E. Locci, ${ }^{28}$ J. Malcles, ${ }^{28}$ J. Rander, ${ }^{28}$ A. Rosowsky, ${ }^{28}$ M. Titov, ${ }^{28}$ S. Baffioni, ${ }^{29}$ F. Beaudette, ${ }^{29}$ P. Busson, ${ }^{29}$ E. Chapon, ${ }^{29}$ C. Charlot ${ }^{29}$ T. Dahms, ${ }^{29}$ M. Dalchenko, ${ }^{29}$ L. Dobrzynski, ${ }^{29}$ N. Filipovic,${ }^{29}$ A. Florent ${ }^{29}$ R. Granier de Cassagnac, ${ }^{29}$ L. Mastrolorenzo, ${ }^{29}$ P. Miné, ${ }^{29}$ I. N. Naranjo, ${ }^{29}$ M. Nguyen,${ }^{29}$ C. Ochando, ${ }^{29}$ G. Ortona, ${ }^{29}$ P. Paganini, ${ }^{29}$ S. Regnard, ${ }^{29}$ R. Salerno, ${ }^{29}$ J. B. Sauvan, ${ }^{29}$ Y. Sirois, ${ }^{29}$ C. Veelken, ${ }^{29}$ Y. Yilmaz, ${ }^{29}$ A. Zabi, ${ }^{29}$ J.-L. Agram, ${ }^{30,0}$ J. Andrea, ${ }^{30}$ A. Aubin, ${ }^{30}$ D. Bloch, ${ }^{30}$ J.-M. Brom, ${ }^{30}$ E. C. Chabert, ${ }^{30}$ C. Collard, ${ }^{30}$ E. Conte, ${ }^{30,0}$ J.-C. Fontaine, ${ }^{30,0}$ D. Gelé, ${ }^{30}$ U. Goerlach, ${ }^{30}$ C. Goetzmann, ${ }^{30}$ A.-C. Le Bihan,${ }^{30}$ K. Skovpen, ${ }^{30}$ P. Van Hove, ${ }^{30}$ S. Gadrat,${ }^{31}$ S. Beauceron, ${ }^{32}$ N. Beaupere, ${ }^{32}$ C. Bernet, ${ }^{32, \mathrm{~h}}$ G. Boudoul,${ }^{32, \mathrm{c}}$ E. Bouvier, ${ }^{32}$ S. Brochet, ${ }^{32}$ C. A. Carrillo Montoya, ${ }^{32}$ J. Chasserat, ${ }^{32}$ R. Chierici, ${ }^{32}$ D. Contardo,${ }^{32, c}$ B. Courbon, ${ }^{32}$ P. Depasse, ${ }^{32}$ H. El Mamouni, ${ }^{32}$ J. Fan, ${ }^{32}$ J. Fay, ${ }^{32}$ S. Gascon, ${ }^{32}$ M. Gouzevitch, ${ }^{32}$ B. Ille, ${ }^{32}$ T. Kurca, ${ }^{32}$ M. Lethuillier, ${ }^{32}$ L. Mirabito, ${ }^{32}$ A. L. Pequegnot, ${ }^{32}$ S. Perries, ${ }^{32}$ J. D. Ruiz Alvarez, ${ }^{32}$

D. Sabes, ${ }^{32}$ L. Sgandurra, ${ }^{32}$ V. Sordini,${ }^{32}$ M. Vander Donckt, ${ }^{32}$ P. Verdier, ${ }^{32}$ S. Viret,${ }^{32}$ H. Xiao, ${ }^{32}$ I. Bagaturia, ${ }^{33, p}$ C. Autermann, ${ }^{34}$ S. Beranek, ${ }^{34}$ M. Bontenackels, ${ }^{34}$ M. Edelhoff, ${ }^{34}$ L. Feld, ${ }^{34}$ A. Heister, ${ }^{34}$ K. Klein, ${ }^{34}$ M. Lipinski, ${ }^{34}$ A. Ostapchuk, ${ }^{34}$ M. Preuten, ${ }^{34}$ F. Raupach, ${ }^{34}$ J. Sammet, ${ }^{34}$ S. Schael, ${ }^{34}$ J. F. Schulte, ${ }^{34}$ H. Weber, ${ }^{34}$ B. Wittmer, ${ }^{34}$ V. Zhukov, ${ }^{34, f}$ M. Ata,${ }^{35}$ M. Brodski, ${ }^{35}$ E. Dietz-Laursonn, ${ }^{35}$ D. Duchardt, ${ }^{35}$ M. Erdmann, ${ }^{35}$ R. Fischer, ${ }^{35}$ A. Güth, ${ }^{35}$ T. Hebbeker, ${ }^{35}$ C. Heidemann, ${ }^{35}$ K. Hoepfner, ${ }^{35}$ D. Klingebiel, ${ }^{35}$ S. Knutzen, ${ }^{35}$ P. Kreuzer, ${ }^{35}$ M. Merschmeyer,${ }^{35}$ A. Meyer, ${ }^{35}$ P. Millet, ${ }^{35}$ M. Olschewski, ${ }^{35}$ K. Padeken, ${ }^{35}$ P. Papacz, ${ }^{35}$ H. Reithler, ${ }^{35}$ S. A. Schmitz, ${ }^{35}$ L. Sonnenschein, ${ }^{35}$ D. Teyssier, ${ }^{35}$ S. Thüer, ${ }^{35}$ M. Weber, ${ }^{35}$ V. Cherepanov, ${ }^{36}$ Y. Erdogan, ${ }^{36}$ G. Flügge, ${ }^{36}$ H. Geenen, ${ }^{36}$ M. Geisler, ${ }^{36}$ W. Haj Ahmad, ${ }^{36}$ F. Hoehle ${ }^{36}$ B. Kargoll, ${ }^{36}$ T. Kress, ${ }^{36}$ Y. Kuessel, ${ }^{36}$ A. Künsken, ${ }^{36}$ J. Lingemann, ${ }^{36, c}$ A. Nowack, ${ }^{36}$ I. M. Nugent, ${ }^{36}$ C. Pistone, ${ }^{36}$ O. Pooth ${ }^{36}$ A. Stahl,${ }^{36}$ M. Aldaya Martin, ${ }^{37}$ I. Asin, ${ }^{37}$ N. Bartosik, ${ }^{37}$ J. Behr, ${ }^{37}$ U. Behrens, ${ }^{37}$ A. J. Bell, ${ }^{37}$ A. Bethani, ${ }^{37}$ K. Borras, ${ }^{37}$ A. Burgmeier, ${ }^{37}$ A. Cakir, ${ }^{37}$ L. Calligaris, ${ }^{37}$ A. Campbell, ${ }^{37}$ S. Choudhury, ${ }^{37}$ F. Costanza, ${ }^{37}$ C. Diez Pardos,${ }^{37}$ G. Dolinska, ${ }^{37}$ S. Dooling, ${ }^{37}$ T. Dorland,${ }^{37}$ G. Eckerlin, ${ }^{37}$ D. Eckstein, ${ }^{37}$ T. Eichhorn,${ }^{37}$ G. Flucke, ${ }^{37}$ J. Garay Garcia, ${ }^{37}$ A. Geiser, ${ }^{37}$ A. Gizhko, ${ }^{37}$ P. Gunnellini, ${ }^{37}$ J. Hauk,${ }^{37}$ M. Hempel, ${ }^{37, q}$ H. Jung, ${ }^{37}$ A. Kalogeropoulos,${ }^{37}$ O. Karacheban, ${ }^{37, q}$ M. Kasemann, ${ }^{37}$ P. Katsas,${ }^{37}$ J. Kieseler, ${ }^{37}$ C. Kleinwort, ${ }^{37}$ I. Korol, ${ }^{37}$ D. Krücker, ${ }^{37}$ W. Lange, ${ }^{37}$ J. Leonard, ${ }^{37}$ K. Lipka, ${ }^{37}$ A. Lobanov, ${ }^{37}$ W. Lohmann, ${ }^{37, q}$ B. Lutz, ${ }^{37}$ R. Mankel,${ }^{37}$ I. Marfin, ${ }^{37, q}$ I.-A. Melzer-Pellmann, ${ }^{37}$ A. B. Meyer, ${ }^{37}$ G. Mittag, ${ }^{37}$ J. Mnich,${ }^{37}$ A. Mussgiller, ${ }^{37}$ S. Naumann-Emme, ${ }^{37}$ A. Nayak, ${ }^{37}$ E. Ntomari, ${ }^{37}$ H. Perrey, ${ }^{37}$ 
D. Pitzl, ${ }^{37}$ R. Placakyte, ${ }^{37}$ A. Raspereza, ${ }^{37}$ P. M. Ribeiro Cipriano, ${ }^{37}$ B. Roland, ${ }^{37}$ E. Ron, ${ }^{37}$ M. Ö. Sahin, ${ }^{37}$

J. Salfeld-Nebgen ${ }^{37}$ P. Saxena, ${ }^{37}$ T. Schoerner-Sadenius, ${ }^{37}$ M. Schröder, ${ }^{37}$ C. Seitz, ${ }^{37}$ S. Spannagel, ${ }^{37}$

A. D. R. Vargas Trevino, ${ }^{37}$ R. Walsh,${ }^{37}$ C. Wissing, ${ }^{37}$ V. Blobel,${ }^{38}$ M. Centis Vignali, ${ }^{38}$ A. R. Draeger, ${ }^{38}$ J. Erfle, ${ }^{38}$ E. Garutti, ${ }^{38}$ K. Goebel, ${ }^{38}$ M. Görner, ${ }^{38}$ J. Haller, ${ }^{38}$ M. Hoffmann, ${ }^{38}$ R. S. Höing, ${ }^{38}$ A. Junkes, ${ }^{38}$ H. Kirschenmann, ${ }^{38}$ R. Klanner, ${ }^{38}$ R. Kogler, ${ }^{38}$ T. Lapsien, ${ }^{38}$ T. Lenz, ${ }^{38}$ I. Marchesini, ${ }^{38}$ D. Marconi, ${ }^{38}$ J. Ott, ${ }^{38}$ T. Peiffer, ${ }^{38}$ A. Perieanu, ${ }^{38}$ N. Pietsch, ${ }^{38}$ J. Poehlsen, ${ }^{38}$ T. Poehlsen, ${ }^{38}$ D. Rathjens, ${ }^{38}$ C. Sander,${ }^{38}$ H. Schettler, ${ }^{38}$ P. Schleper, ${ }^{38}$ E. Schlieckau, ${ }^{38}$ A. Schmidt,${ }^{38}$ M. Seidel,${ }^{38}$ V. Sola,${ }^{38}$ H. Stadie,${ }^{38}$ G. Steinbrück, ${ }^{38}$ D. Troendle,${ }^{38}$ E. Usai, ${ }^{38}$ L. Vanelderen,${ }^{38}$ A. Vanhoefer,${ }^{38}$ C. Barth, ${ }^{39}$ C. Baus, ${ }^{39}$ J. Berger, ${ }^{39}$ C. Böser, ${ }^{39}$ E. Butz, ${ }^{39}$ T. Chwalek, ${ }^{39}$ W. De Boer,${ }^{39}$ A. Descroix,${ }^{39}$ A. Dierlamm, ${ }^{39}$ M. Feindt,${ }^{39}$ F. Frensch, ${ }^{39}$ M. Giffels, ${ }^{39}$ A. Gilbert, ${ }^{39}$ F. Hartmann,,${ }^{39, \mathrm{c}}$ T. Hauth, ${ }^{39}$ U. Husemann, ${ }^{39}$ I. Katkov, ${ }^{39, f}$ A. Kornmayer, ${ }^{39, \mathrm{c}}$ P. Lobelle Pardo, ${ }^{39}$ M. U. Mozer, ${ }^{39}$ T. Müller, ${ }^{39}$ Th. Müller, ${ }^{39}$ A. Nürnberg,,${ }^{39}$ G. Quast, ${ }^{39}$ K. Rabbertz, ${ }^{39}$ S. Röcker, ${ }^{39}$ H. J. Simonis, ${ }^{39}$ F. M. Stober, ${ }^{39}$ R. Ulrich, ${ }^{39}$ J. Wagner-Kuhr, ${ }^{39}$ S. Wayand, ${ }^{39}$ T. Weiler, ${ }^{39}$ R. Wolf, ${ }^{39}$ G. Anagnostou, ${ }^{40}$ G. Daskalakis, ${ }^{40}$ T. Geralis, ${ }^{40}$ V. A. Giakoumopoulou, ${ }^{40}$ A. Kyriakis, ${ }^{40}$ D. Loukas, ${ }^{40}$ A. Markou, ${ }^{40}$ C. Markou, ${ }^{40}$ A. Psallidas, ${ }^{40}$ I. Topsis-Giotis, ${ }^{40}$ A. Agapitos, ${ }^{41}$ S. Kesisoglou, ${ }^{41}$ A. Panagiotou, ${ }^{41}$ N. Saoulidou, ${ }^{41}$ E. Stiliaris, ${ }^{41}$ E. Tziaferi, ${ }^{41}$ X. Aslanoglou, ${ }^{42}$ I. Evangelou, ${ }^{42}$ G. Flouris, ${ }^{42}$ C. Foudas, ${ }^{42}$ P. Kokkas, ${ }^{42}$ N. Manthos,${ }^{42}$

I. Papadopoulos, ${ }^{42}$ E. Paradas, ${ }^{42}$ J. Strologas, ${ }^{42}$ G. Bencze, ${ }^{43}$ C. Hajdu, ${ }^{43}$ P. Hidas, ${ }^{43}$ D. Horvath, ${ }^{43, r}$ F. Sikler, ${ }^{43}$ V. Veszpremi, ${ }^{43}$ G. Vesztergombi, ${ }^{43,}$ A. J. Zsigmond, ${ }^{43}$ N. Beni, ${ }^{44}$ S. Czellar, ${ }^{44}$ J. Karancsi, ${ }^{44, t}$ J. Molnar, ${ }^{44}$ J. Palinkas, ${ }^{44}$ Z. Szillasi, ${ }^{44}$ A. Makovec ${ }^{45}$ P. Raics, ${ }^{45}$ Z. L. Trocsanyi, ${ }^{45}$ B. Ujvari, ${ }^{45}$ S. K. Swain, ${ }^{46}$ S. B. Beri, ${ }^{47}$ V. Bhatnagar, ${ }^{47}$ R. Gupta, ${ }^{47}$ U. Bhawandeep, ${ }^{47}$ A. K. Kalsi ${ }^{47}$ M. Kaur, ${ }^{47}$ R. Kumar, ${ }^{47}$ M. Mittal, ${ }^{47}$ N. Nishu, ${ }^{47}$ J. B. Singh,${ }^{47}$ Ashok Kumar, ${ }^{48}$ Arun Kumar, ${ }^{48}$ S. Ahuja, ${ }^{48}$ A. Bhardwaj, ${ }^{48}$ B. C. Choudhary, ${ }^{48}$ A. Kumar, ${ }^{48}$ S. Malhotra, ${ }^{48}$ M. Naimuddin, ${ }^{48}$ K. Ranjan, ${ }^{48}$ V. Sharma, ${ }^{48}$ S. Banerjee, ${ }^{49}$ S. Bhattacharya ${ }^{49}$ K. Chatterjee, ${ }^{49}$ S. Dutta, ${ }^{49}$ B. Gomber, ${ }^{49}$ Sa. Jain, ${ }^{49}$ Sh. Jain, ${ }^{49}$ R. Khurana, ${ }^{49}$ A. Modak ${ }^{49}$ S. Mukherjee, ${ }^{49}$ D. Roy, ${ }^{49}$ S. Sarkar, ${ }^{49}$ M. Sharan, ${ }^{49}$ A. Abdulsalam, ${ }^{50}$ D. Dutta,${ }^{50}$ V. Kumar, ${ }^{50}$ A. K. Mohanty, ${ }^{50, c}$ L. M. Pant, ${ }^{50}$ P. Shukla ${ }^{50}$ A. Topkar, ${ }^{50}$ T. Aziz,${ }^{51}$ S. Banerjee, ${ }^{51}$ S. Bhowmik, ${ }^{51, u}$ R. M. Chatterjee, ${ }^{51}$ R. K. Dewanjee, ${ }^{51}$ S. Dugad,${ }^{51}$ S. Ganguly, ${ }^{51}$ S. Ghosh, ${ }^{51}$ M. Guchait, ${ }^{51}$ A. Gurtu, ${ }^{51, v}$ G. Kole, ${ }^{51}$ S. Kumar, ${ }^{51}$ M. Maity, ${ }^{51, u}$ G. Majumder, ${ }^{51}$ K. Mazumdar, ${ }^{51}$ G. B. Mohanty ${ }^{51}$ B. Parida, ${ }^{51}$ K. Sudhakar,${ }^{51}$ N. Wickramage,${ }^{51, w}$ S. Sharma, ${ }^{52}$ H. Bakhshiansohi, ${ }^{53}$ H. Behnamian, ${ }^{53}$ S. M. Etesami, ${ }^{53, x}$ A. Fahim, ${ }^{53, y}$ R. Goldouzian, ${ }^{53}$ M. Khakzad, ${ }^{53}$

M. Mohammadi Najafabadi, ${ }^{53}$ M. Naseri, ${ }^{53}$ S. Paktinat Mehdiabadi, ${ }^{53}$ F. Rezaei Hosseinabadi, ${ }^{53}$ B. Safarzadeh, ${ }^{53, z}$ M. Zeinali, ${ }^{53}$ M. Felcini, ${ }^{54}$ M. Grunewald, ${ }^{54}$ M. Abbrescia, ${ }^{55 a, 55 b}$ C. Calabria, ${ }^{55 a, 55 b}$ S. S. Chhibra, ${ }^{55 a, 55 b}$ A. Colaleo, ${ }^{55 a}$ D. Creanza, ${ }^{55 a, 55 c}$ L. Cristella, ${ }^{55 a, 55 b}$ N. De Filippis, ${ }^{55 a, 55 c}$ M. De Palma,${ }^{55 a, 55 b}$ L. Fiore, ${ }^{55 a}$ G. Iaselli, ${ }^{55 a, 55 c}$ G. Maggi, ${ }^{55 a, 55 c}$ M. Maggi, ${ }^{55 a}$ S. My, ${ }^{55 a, 55 c}$ S. Nuzzo, ${ }^{55 a, 55 b}$ A. Pompili,${ }^{55 a, 55 b}$ G. Pugliese,${ }^{55 a, 55 c}$ R. Radogna, ${ }^{55 a, 55 b, c}$ G. Selvaggi, ${ }^{55 a, 55 b}$ A. Sharma, ${ }^{55 \mathrm{a}}$ L. Silvestris, ${ }^{55 \mathrm{a}, \mathrm{c}}$ R. Venditti,${ }^{55,55 \mathrm{~b}}$ P. Verwilligen, ${ }^{55 \mathrm{a}}$ G. Abbiendi, ${ }^{56 \mathrm{a}}$ A. C. Benvenuti, ${ }^{56 \mathrm{a}}$ D. Bonacorsi, ${ }^{56 a, 56 b}$ S. Braibant-Giacomelli, ${ }^{56,56 \mathrm{~b}}$ L. Brigliadori, ${ }^{56,56 \mathrm{~b}}$ R. Campanini, ${ }^{56,56 \mathrm{~b}}$ P. Capiluppi, ${ }^{56,56 \mathrm{~b}}$ A. Castro, ${ }^{56 \mathrm{a}, 56 \mathrm{~b}}$ F. R. Cavallo, ${ }^{56 \mathrm{a}}$ G. Codispoti, ${ }^{56,56 \mathrm{~b}}$ M. Cuffiani, ${ }^{56,56 \mathrm{~b}}$ G. M. Dallavalle, ${ }^{56 \mathrm{a}}$ F. Fabbri, ${ }^{56 \mathrm{a}}$ A. Fanfani, ${ }^{56 \mathrm{a}, 56 \mathrm{~b}}$ D. Fasanella, ${ }^{56,56 \mathrm{~b}}$ P. Giacomelli, ${ }^{56 \mathrm{a}}$ C. Grandi, ${ }^{56 \mathrm{a}}$ L. Guiducci, ${ }^{56,56 \mathrm{~b}}$ S. Marcellini, ${ }^{56 \mathrm{a}}$ G. Masetti, ${ }^{56 \mathrm{a}}$ A. Montanari, ${ }^{56 \mathrm{a}}$ F. L. Navarria, ${ }^{56 a, 56 \mathrm{~b}}$ A. Perrotta, ${ }^{56 \mathrm{a}}$ A. M. Rossi, ${ }^{56 a, 56 \mathrm{~b}}$ T. Rovelli, ${ }^{56 \mathrm{a}, 56 \mathrm{~b}}$ G. P. Siroli, ${ }^{56,56 \mathrm{~b}}$ N. Tosi, ${ }^{56,56 \mathrm{~b}}$ R. Travaglini, ${ }^{56 \mathrm{a}, 56 \mathrm{~b}}$ S. Albergo, ${ }^{57 \mathrm{a}, 57 \mathrm{~b}}$ G. Cappello, ${ }^{57 \mathrm{a}}$ M. Chiorboli, ${ }^{57,57 b}$ S. Costa,${ }^{57,57 b}$ F. Giordano, ${ }^{57 a, 57 c, c}$ R. Potenza,${ }^{57 a, 57 b}$ A. Tricomi, ${ }^{57 a, 57 b}$ C. Tuve, ${ }^{57 a, 57 b}$ G. Barbagli, ${ }^{58 \mathrm{a}}$ V. Ciulli, ${ }^{58 \mathrm{a}, 58 \mathrm{~b}}$ C. Civinini, ${ }^{58 \mathrm{a}}$ R. D’Alessandro, ${ }^{58 \mathrm{a}, 58 \mathrm{~b}}$ E. Focardi, ${ }^{58 \mathrm{a}, 58 \mathrm{~b}}$ E. Gallo, ${ }^{58 \mathrm{a}}$ S. Gonzi, ${ }^{58 \mathrm{a}, 58 \mathrm{~b}}$ V. Gori, ${ }^{58 a, 58 b}$ P. Lenzi, ${ }^{58 a, 58 b}$ M. Meschini, ${ }^{58 \mathrm{a}}$ S. Paoletti, ${ }^{58 \mathrm{a}}$ G. Sguazzoni, ${ }^{58 \mathrm{a}}$ A. Tropiano, ${ }^{58 \mathrm{a}, 58 \mathrm{~b}}$ L. Benussi, ${ }^{59}$ S. Bianco, ${ }^{59}$ F. Fabbri,${ }^{59}$ D. Piccolo, ${ }^{59}$ R. Ferretti, ${ }^{60 \mathrm{a}, 60 \mathrm{~b}}$ F. Ferro, ${ }^{60 \mathrm{a}}$ M. Lo Vetere, ${ }^{60 \mathrm{a}, 60 \mathrm{~b}}$ E. Robutti, ${ }^{60 \mathrm{a}}$ S. Tosi,${ }^{60 \mathrm{a}, 60 \mathrm{~b}}$ M. E. Dinardo, ${ }^{61 \mathrm{a}, 61 \mathrm{~b}}$ S. Fiorendi, ${ }^{61 a, 61 b}$ S. Gennai ${ }^{61 a, c}$ R. Gerosa, ${ }^{61 a, 61 b, c}$ A. Ghezzi, ${ }^{61 a, 61 b}$ P. Govoni, ${ }^{61 a, 61 b}$ M. T. Lucchini, ${ }^{61 a, 61 b, c}$ S. Malvezzi, ${ }^{61 a}$ R. A. Manzoni ${ }^{61 \mathrm{a}, 61 \mathrm{~b}}$ A. Martelli, ${ }^{61 \mathrm{a}, 61 \mathrm{~b}}$ B. Marzocchi, ${ }^{61 \mathrm{a}, 61 \mathrm{~b}, \mathrm{c}}$ D. Menasce, ${ }^{61 \mathrm{a}}$ L. Moroni, ${ }^{61 \mathrm{a}}$ M. Paganoni, ${ }^{61 \mathrm{a}, 61 \mathrm{~b}}$ D. Pedrini, ${ }^{61 \mathrm{a}}$ S. Ragazzi ${ }^{61 \mathrm{a}, 61 \mathrm{~b}}$ N. Redaelli, ${ }^{61 \mathrm{a}}$ T. Tabarelli de Fatis, ${ }^{61 \mathrm{a}, 61 \mathrm{~b}}$ S. Buontempo ${ }^{62 \mathrm{a}}$ N. Cavallo, ${ }^{62 \mathrm{a}, 62 \mathrm{c}}$ S. Di Guida, ${ }^{62 \mathrm{a}, 62 \mathrm{~d}, \mathrm{c}}$ F. Fabozzi, ${ }^{62 a, 62 c}$ A. O. M. Iorio, ${ }^{62 a, 62 b}$ L. Lista, ${ }^{62 a}$ S. Meola,${ }^{62 a, 62 d, c}$ M. Merola, ${ }^{62 a}$ P. Paolucci, ${ }^{62 a, c}$ P. Azzi, ${ }^{63 a}$ N. Bacchetta, ${ }^{63 a}$ M. Bellato, ${ }^{63 a}$ D. Bisello, ${ }^{63 a, 63 b}$ R. Carlin, ${ }^{63 a, 63 b}$ P. Checchia, ${ }^{63 a}$ M. Dall’Osso, ${ }^{63 a, 63 b}$ T. Dorigo, ${ }^{63 a}$ S. Fantinel, ${ }^{63 a}$

F. Gasparini ${ }^{63 a, 63 b}$ U. Gasparini, ${ }^{63 a, 63 b}$ F. Gonella ${ }^{63 a}$ A. Gozzelino, ${ }^{63 a}$ S. Lacaprara, ${ }^{63 a}$ M. Margoni, ${ }^{63 a, 63 b}$ A. T. Meneguzzo, ${ }^{63 a, 63 b}$ F. Montecassiano, ${ }^{63 a}$ J. Pazzini, ${ }^{63 a, 63 b}$ N. Pozzobon, ${ }^{63 a, 63 b}$ P. Ronchese, ${ }^{63 a, 63 b}$ F. Simonetto, ${ }^{63 a, 63 b}$ E. Torassa, ${ }^{63 a}$ M. Tosi ${ }^{63 a, 63 b}$ P. Zotto, ${ }^{63 a, 63 b}$ A. Zucchetta, ${ }^{63 a, 63 b}$ M. Gabusi, ${ }^{64 a, 64 b}$ S. P. Ratti, ${ }^{64 a, 64 b}$ V. Re,${ }^{64 a}$ C. Riccardi, ${ }^{64 a, 64 b}$ P. Salvini, ${ }^{64 a}$ P. Vitulo, ${ }^{64 a, 64 b}$ M. Biasini, ${ }^{65 a, 65 b}$ G. M. Bilei ${ }^{65 a}$ D. Ciangottini, ${ }^{65 a, 65 b, c}$ L. Fanò, ${ }^{65 a, 65 b}$ P. Lariccia, ${ }^{65 a, 65 b}$ G. Mantovani, ${ }^{65 a, 65 b}$ M. Menichelli, ${ }^{65 a}$ A. Saha, ${ }^{65 a}$ A. Santocchia, ${ }^{65 a, 65 b}$ A. Spiezia, ${ }^{65 a, 65 b, c}$ K. Androsov, ${ }^{66 a, a a}$ P. Azzurri, ${ }^{66 a}$ G. Bagliesi, ${ }^{66 a}$ J. Bernardini, ${ }^{66 a}$ T. Boccali, ${ }^{66 a}$ G. Broccolo, ${ }^{66 a, 66 c}$ R. Castaldi, ${ }^{66 a}$ M. A. Ciocci, ${ }^{66 a, a a}$ R. Dell'Orso, ${ }^{66 a}$ 
S. Donato, ${ }^{66 a, 66 c, c}$ G. Fedi, ${ }^{66 a}$ F. Fiori, ${ }^{66 a, 66 c}$ L. Foà ${ }^{66 a, 66 c}$ A. Giassi, ${ }^{66 a}$ M. T. Grippo, ${ }^{66 a, a a}$ F. Ligabue, ${ }^{66 a, 66 c}$ T. Lomtadze, ${ }^{66 a}$

L. Martini ${ }^{66 a, 66 b}$ A. Messineo, ${ }^{66 a, 66 b}$ C. S. Moon, ${ }^{66 a, b b}$ F. Palla, ${ }^{66 a, c}$ A. Rizzi, ${ }^{66 a, 66 b}$ A. Savoy-Navarro, ${ }^{66 a, c c}$ A. T. Serban, ${ }^{66 a}$

P. Spagnolo, ${ }^{66 a}$ P. Squillacioti, ${ }^{66 a, a a}$ R. Tenchini, ${ }^{66 a}$ G. Tonelli, ${ }^{66 a, 66 b}$ A. Venturi, ${ }^{66 a}$ P. G. Verdini, ${ }^{66 a}$ C. Vernieri, ${ }^{66 a, 66 c}$

L. Barone ${ }^{67 a, 67 b}$ F. Cavallari, ${ }^{67 a}$ G. D'imperio, ${ }^{67 a, 67 b}$ D. Del Re,${ }^{67,67 b}$ M. Diemoz, ${ }^{67 a}$ C. Jorda,${ }^{67 a}$ E. Longo, ${ }^{67 a, 67 b}$

F. Margaroli, ${ }^{67 a, 67 b}$ P. Meridiani, ${ }^{67 a}$ F. Micheli, ${ }^{67 a, 67 b, c}$ G. Organtini, ${ }^{67 a, 67 b}$ R. Paramatti, ${ }^{67 a}$ S. Rahatlou ${ }^{67 a, 67 b}$ C. Rovelli, ${ }^{67 a}$

F. Santanastasio, ${ }^{67 a, 67 b}$ L. Soffi,${ }^{67 a, 67 b}$ P. Traczyk,${ }^{67 a, 67 b, c}$ N. Amapane, ${ }^{68 a, 68 b}$ R. Arcidiacono, ${ }^{68 a, 68 c}$ S. Argiro, ${ }^{68 a, 68 b}$

M. Arneodo, ${ }^{68 a, 68 c}$ R. Bellan, ${ }^{68 a, 68 b}$ C. Biino, ${ }^{68 a}$ N. Cartiglia, ${ }^{68 a}$ S. Casasso, ${ }^{68 a, 68 b, c}$ M. Costa, ${ }^{68 a, 68 b}$ R. Covarelli, ${ }^{68 a}$

A. Degano, ${ }^{68 a, 68 b}$ N. Demaria, ${ }^{68 a}$ L. Finco, ${ }^{68 a, 68 b, c}$ C. Mariotti, ${ }^{68 a}$ S. Maselli, ${ }^{68 a}$ E. Migliore, ${ }^{68 a, 68 b}$ V. Monaco, ${ }^{68 a, 68 b}$

M. Musich, ${ }^{68 \mathrm{a}}$ M. M. Obertino, ${ }^{68 a, 68 \mathrm{c}}$ L. Pacher, ${ }^{68 \mathrm{a}, 68 \mathrm{~b}}$ N. Pastrone, ${ }^{68 \mathrm{a}}$ M. Pelliccioni, ${ }^{68 \mathrm{a}}$ G. L. Pinna Angioni, ${ }^{68,68 \mathrm{~b}}$

A. Potenza ${ }^{68 a, 68 b}$ A. Romero, ${ }^{68,68 b}$ M. Ruspa,${ }^{68 a, 68 c}$ R. Sacchi, ${ }^{68 a, 68 b}$ A. Solano, ${ }^{68 a, 68 b}$ A. Staiano, ${ }^{68 \mathrm{a}}$ U. Tamponi, ${ }^{68 \mathrm{a}}$

S. Belforte, ${ }^{69 a}$ V. Candelise,${ }^{69 a, 69 b, c}$ M. Casarsa ${ }^{69 a}$ F. Cossutti, ${ }^{69 a}$ G. Della Ricca, ${ }^{69 a, 69 b}$ B. Gobbo, ${ }^{69 a}$ C. La Licata, ${ }^{69 a, 69 b}$ M. Marone ${ }^{69 a, 69 b}$ A. Schizzi, ${ }^{69,69 b}$ T. Umer, ${ }^{69 a, 69 b}$ A. Zanetti, ${ }^{69 a}$ S. Chang,${ }^{70}$ A. Kropivnitskaya ${ }^{70}$ S. K. Nam ${ }^{70}$ D. H. Kim,${ }^{71}$

G. N. Kim, ${ }^{71}$ M. S. Kim, ${ }^{71}$ D. J. Kong, ${ }^{71}$ S. Lee, ${ }^{71}$ Y. D. Oh, ${ }^{71}$ H. Park, ${ }^{71}$ A. Sakharov, ${ }^{71}$ D. C. Son, ${ }^{71}$ T. J. Kim, ${ }^{72}$ M. S. Ryu ${ }^{72}$ J. Y. Kim, ${ }^{73}$ D. H. Moon, ${ }^{73}$ S. Song, ${ }^{73}$ S. Choi,${ }^{74}$ D. Gyun, ${ }^{74}$ B. Hong, ${ }^{74}$ M. Jo, ${ }^{74}$ H. Kim, ${ }^{74}$ Y. Kim, ${ }^{74}$ B. Lee,${ }^{74}$ K. S. Lee, ${ }^{74}$ S. K. Park,${ }^{74}$ Y. Roh, ${ }^{74}$ H. D. Yoo, ${ }^{75}$ M. Choi,${ }^{76}$ J. H. Kim,${ }^{76}$ I. C. Park,${ }^{76}$ G. Ryu ${ }^{76}$ Y. Choi, ${ }^{77}$ Y. K. Choi,${ }^{77}$ J. Goh, ${ }^{77}$ D. Kim, ${ }^{77}$ E. Kwon, ${ }^{77}$ J. Lee, ${ }^{77}$ I. Yu, ${ }^{77}$ A. Juodagalvis, ${ }^{78}$ J. R. Komaragiri, ${ }^{79}$ M. A. B. Md Ali, ${ }^{79}$

E. Casimiro Linares ${ }^{80}$ H. Castilla-Valdez ${ }^{80}$ E. De La Cruz-Burelo, ${ }^{80}$ I. Heredia-de La Cruz, ${ }^{80}$ A. Hernandez-Almada, ${ }^{80}$

R. Lopez-Fernandez ${ }^{80}$ A. Sanchez-Hernandez, ${ }^{80}$ S. Carrillo Moreno, ${ }^{81}$ F. Vazquez Valencia, ${ }^{81}$ I. Pedraza, ${ }^{82}$

H. A. Salazar Ibarguen, ${ }^{82}$ A. Morelos Pineda, ${ }^{83}$ D. Krofcheck, ${ }^{84}$ P. H. Butler, ${ }^{85}$ S. Reucroft, ${ }^{85}$ A. Ahmad ${ }^{86}$ M. Ahmad, ${ }^{86}$ Q. Hassan, ${ }^{86}$ H. R. Hoorani, ${ }^{86}$ W. A. Khan, ${ }^{86}$ T. Khurshid ${ }^{86}$ M. Shoaib,${ }^{86}$ H. Bialkowska, ${ }^{87}$ M. Bluj, ${ }^{87}$ B. Boimska, ${ }^{87}$ T. Frueboes, ${ }^{87}$ M. Górski, ${ }^{87}$ M. Kazana ${ }^{87}$ K. Nawrocki, ${ }^{87}$ K. Romanowska-Rybinska, ${ }^{87}$ M. Szleper, ${ }^{87}$ P. Zalewski, ${ }^{87}$ G. Brona,${ }^{88}$ K. Bunkowski, ${ }^{88}$ M. Cwiok ${ }^{88}$ W. Dominik ${ }^{88}$ K. Doroba, ${ }^{88}$ A. Kalinowski, ${ }^{88}$ M. Konecki, ${ }^{88}$ J. Krolikowski, ${ }^{88}$ M. Misiura, ${ }^{88}$ M. Olszewski, ${ }^{88}$ P. Bargassa, ${ }^{89}$ C. Beirão Da Cruz E Silva, ${ }^{89}$ P. Faccioli, ${ }^{89}$ P. G. Ferreira Parracho, ${ }^{89}$

M. Gallinaro, ${ }^{89}$ L. Lloret Iglesias, ${ }^{89}$ F. Nguyen, ${ }^{89}$ J. Rodrigues Antunes, ${ }^{89}$ J. Seixas, ${ }^{89}$ J. Varela, ${ }^{89}$ P. Vischia, ${ }^{89}$ S. Afanasiev, ${ }^{90}$ P. Bunin, ${ }^{90}$ M. Gavrilenko, ${ }^{90}$ I. Golutvin, ${ }^{90}$ I. Gorbunov,${ }^{90}$ A. Kamenev, ${ }^{90}$ V. Karjavin, ${ }^{90}$ V. Konoplyanikov, ${ }^{90}$ A. Lanev, ${ }^{90}$ A. Malakhov, ${ }^{90}$ V. Matveev, ${ }^{90, d d}$ P. Moisenz, ${ }^{90}$ V. Palichik, ${ }^{90}$ V. Perelygin, ${ }^{90}$ S. Shmatov, ${ }^{90}$ N. Skatchkov, ${ }^{90}$ V. Smirnov, ${ }^{90}$ A. Zarubin, ${ }^{90}$ V. Golovtsov, ${ }^{91}$ Y. Ivanov, ${ }^{91}$ V. Kim,,${ }^{91, e e}$ E. Kuznetsova, ${ }^{91}$ P. Levchenko, ${ }^{91}$ V. Murzin, ${ }^{91}$ V. Oreshkin, ${ }^{91}$ I. Smirnov, ${ }^{91}$ V. Sulimov,${ }^{91}$ L. Uvarov,${ }^{91}$ S. Vavilov, ${ }^{91}$ A. Vorobyev, ${ }^{91}$ An. Vorobyev, ${ }^{91}$ Yu. Andreev,${ }^{92}$ A. Dermenev, ${ }^{92}$ S. Gninenko, ${ }^{92}$ N. Golubev, ${ }^{92}$ M. Kirsanov, ${ }^{92}$ N. Krasnikov, ${ }^{92}$ A. Pashenkov, ${ }^{92}$ D. Tlisov, ${ }^{92}$ A. Toropin,${ }^{92}$ V. Epshteyn, ${ }^{93}$ V. Gavrilov, ${ }^{93}$ N. Lychkovskaya, ${ }^{93}$ V. Popov, ${ }^{93}$ I. Pozdnyakov, ${ }^{93}$ G. Safronov, ${ }^{93}$ S. Semenov,${ }^{93}$ A. Spiridonov, ${ }^{93}$ V. Stolin, ${ }^{93}$ E. Vlasov, ${ }^{93}$ A. Zhokin, ${ }^{93}$ V. Andreev, ${ }^{94}$ M. Azarkin, ${ }^{94}$ I. Dremin, ${ }^{94}$ M. Kirakosyan, ${ }^{94}$ A. Leonidov, ${ }^{94}$ G. Mesyats, ${ }^{94}$ S. V. Rusakov, ${ }^{94}$ A. Vinogradov,${ }^{94}$ A. Belyaev,${ }^{95}$ E. Boos, ${ }^{95}$ M. Dubinin, ${ }^{95, f f}$ L. Dudko, ${ }^{95}$ A. Ershov, ${ }^{95}$ A. Gribushin, ${ }^{95}$ V. Klyukhin, ${ }^{95}$ O. Kodolova, ${ }^{95}$ I. Lokhtin,${ }^{95}$ S. Obraztsov, ${ }^{95}$ S. Petrushanko, ${ }^{95}$ V. Savrin, ${ }^{95}$ A. Snigirev,${ }^{95}$ I. Azhgirey,${ }^{96}$ I. Bayshev, ${ }^{96}$ S. Bitioukov, ${ }^{96}$ V. Kachanov, ${ }^{96}$ A. Kalinin, ${ }^{96}$ D. Konstantinov,${ }^{96}$ V. Krychkine, ${ }^{96}$ V. Petrov, ${ }^{96}$ R. Ryutin, ${ }^{96}$ A. Sobol, ${ }^{96}$ L. Tourtchanovitch, ${ }^{96}$ S. Troshin, ${ }^{96}$ N. Tyurin, ${ }^{96}$ A. Uzunian, ${ }^{96}$ A. Volkov, ${ }^{96}$ P. Adzic, ${ }^{97, g g}$ M. Ekmedzic, ${ }^{97}$ J. Milosevic,${ }^{97}$ V. Rekovic,${ }^{97}$ J. Alcaraz Maestre, ${ }^{98}$ C. Battilana, ${ }^{98}$ E. Calvo, ${ }^{98}$ M. Cerrada, ${ }^{98}$ M. Chamizo Llatas, ${ }^{98}$ N. Colino, ${ }^{98}$ B. De La Cruz,${ }^{98}$ A. Delgado Peris, ${ }^{98}$ D. Domínguez Vázquez,${ }^{98}$

A. Escalante Del Valle, ${ }^{98}$ C. Fernandez Bedoya, ${ }^{98}$ J. P. Fernández Ramos, ${ }^{98}$ J. Flix, ${ }^{98}$ M. C. Fouz, ${ }^{98}$ P. Garcia-Abia, ${ }^{98}$ O. Gonzalez Lopez, ${ }^{98}$ S. Goy Lopez, ${ }^{98}$ J. M. Hernandez, ${ }^{98}$ M. I. Josa, ${ }^{98}$ E. Navarro De Martino, ${ }^{98}$

A. Pérez-Calero Yzquierdo, ${ }^{98}$ J. Puerta Pelayo, ${ }^{98}$ A. Quintario Olmeda, ${ }^{98}$ I. Redondo, ${ }^{98}$ L. Romero, ${ }^{98}$ M. S. Soares,${ }^{98}$ C. Albajar, ${ }^{99}$ J. F. de Trocóniz, ${ }^{99}$ M. Missiroli, ${ }^{99}$ D. Moran, ${ }^{99}$ H. Brun, ${ }^{100}$ J. Cuevas, ${ }^{100}$ J. Fernandez Menendez, ${ }^{100}$ S. Folgueras, ${ }^{100}$ I. Gonzalez Caballero, ${ }^{100}$ J. A. Brochero Cifuentes, ${ }^{101}$ I. J. Cabrillo, ${ }^{101}$ A. Calderon, ${ }^{101}$

J. Duarte Campderros, ${ }^{101}$ M. Fernandez, ${ }^{101}$ G. Gomez, ${ }^{101}$ A. Graziano, ${ }^{101}$ A. Lopez Virto, ${ }^{101}$ J. Marco, ${ }^{101}$ R. Marco, ${ }^{101}$ C. Martinez Rivero, ${ }^{101}$ F. Matorras, ${ }^{101}$ F. J. Munoz Sanchez,${ }^{101}$ J. Piedra Gomez, ${ }^{101}$ T. Rodrigo, ${ }^{101}$

A. Y. Rodríguez-Marrero, ${ }^{101}$ A. Ruiz-Jimeno, ${ }^{101}$ L. Scodellaro, ${ }^{101}$ I. Vila, ${ }^{101}$ R. Vilar Cortabitarte, ${ }^{101}$ D. Abbaneo, ${ }^{102}$ E. Auffray, ${ }^{102}$ G. Auzinger, ${ }^{102}$ M. Bachtis,${ }^{102}$ P. Baillon, ${ }^{102}$ A. H. Ball, ${ }^{102}$ D. Barney, ${ }^{102}$ A. Benaglia, ${ }^{102}$ J. Bendavid, ${ }^{102}$ L. Benhabib ${ }^{102}$ J. F. Benitez,${ }^{102}$ P. Bloch, ${ }^{102}$ A. Bocci, ${ }^{102}$ A. Bonato, ${ }^{102}$ O. Bondu, ${ }^{102}$ C. Botta, ${ }^{102}$ H. Breuker, ${ }^{102}$

T. Camporesi, ${ }^{102}$ G. Cerminara, ${ }^{102}$ S. Colafranceschi,${ }^{102, \text { hh }}$ M. D'Alfonso, ${ }^{102}$ D. d'Enterria, ${ }^{102}$ A. Dabrowski, ${ }^{102}$ A. David, ${ }^{102}$ F. De Guio, ${ }^{102}$ A. De Roeck, ${ }^{102}$ S. De Visscher, ${ }^{102}$ E. Di Marco, ${ }^{102}$ M. Dobson, ${ }^{102}$ M. Dordevic, ${ }^{102}$ B. Dorney, ${ }^{102}$ 
N. Dupont-Sagorin, ${ }^{102}$ A. Elliott-Peisert, ${ }^{102}$ G. Franzoni, ${ }^{102}$ W. Funk,${ }^{102}$ D. Gigi, ${ }^{102}$ K. Gill, ${ }^{102}$ D. Giordano, ${ }^{102}$ M. Girone,${ }^{102}$ F. Glege,${ }^{102}$ R. Guida, ${ }^{102}$ S. Gundacker, ${ }^{102}$ M. Guthoff, ${ }^{102}$ J. Hammer, ${ }^{102}$ M. Hansen, ${ }^{102}$ P. Harris, ${ }^{102}$ J. Hegeman, ${ }^{102}$ V. Innocente, ${ }^{102}$ P. Janot, ${ }^{102}$ K. Kousouris, ${ }^{102}$ K. Krajczar, ${ }^{102}$ P. Lecoq,${ }^{102}$ C. Lourenço, ${ }^{102}$ N. Magini, ${ }^{102}$ L. Malgeri, ${ }^{102}$ M. Mannelli, ${ }^{102}$ J. Marrouche, ${ }^{102}$ L. Masetti, ${ }^{102}$ F. Meijers, ${ }^{102}$ S. Mersi, ${ }^{102}$ E. Meschi, ${ }^{102}$ F. Moortgat,${ }^{102}$ S. Morovic, ${ }^{102}$ M. Mulders, ${ }^{102}$ L. Orsini, ${ }^{102}$ L. Pape, ${ }^{102}$ E. Perez, ${ }^{102}$ A. Petrilli, ${ }^{102}$ G. Petrucciani, ${ }^{102}$ A. Pfeiffer, ${ }^{102}$ M. Pimiä, ${ }^{102}$ D. Piparo, ${ }^{102}$ M. Plagge, ${ }^{102}$ A. Racz, ${ }^{102}$ G. Rolandi, ${ }^{102, i i}$ M. Rovere ${ }^{102}$ H. Sakulin, ${ }^{102}$ C. Schäfer, ${ }^{102}$ C. Schwick, ${ }^{102}$ A. Sharma,${ }^{102}$

P. Siegrist, ${ }^{102}$ P. Silva, ${ }^{102}$ M. Simon, ${ }^{102}$ P. Sphicas, ${ }^{102, j j}$ D. Spiga, ${ }^{102}$ J. Steggemann, ${ }^{102}$ B. Stieger, ${ }^{102}$ M. Stoye, ${ }^{102}$ Y. Takahashi, ${ }^{102}$ D. Treille, ${ }^{102}$ A. Tsirou, ${ }^{102}$ G. I. Veres, ${ }^{102, s}$ N. Wardle, ${ }^{102}$ H. K. Wöhri, ${ }^{102}$ H. Wollny, ${ }^{102}$ W. D. Zeuner, ${ }^{102}$ W. Bertl, ${ }^{103}$ K. Deiters, ${ }^{103}$ W. Erdmann, ${ }^{103}$ R. Horisberger, ${ }^{103}$ Q. Ingram, ${ }^{103}$ H. C. Kaestli, ${ }^{103}$ D. Kotlinski, ${ }^{103}$ U. Langenegger, ${ }^{103}$ D. Renker, ${ }^{103}$ T. Rohe, ${ }^{103}$ F. Bachmair, ${ }^{104}$ L. Bäni, ${ }^{104}$ L. Bianchini, ${ }^{104}$ M. A. Buchmann, ${ }^{104}$ B. Casal, ${ }^{104}$ N. Chanon, ${ }^{104}$ G. Dissertori, ${ }^{104}$ M. Dittmar, ${ }^{104}$ M. Donegà, ${ }^{104}$ M. Dünser, ${ }^{104}$ P. Eller, ${ }^{104}$ C. Grab, ${ }^{104}$ D. Hits, ${ }^{104}$ J. Hoss, ${ }^{104}$ W. Lustermann, ${ }^{104}$ B. Mangano, ${ }^{104}$ A. C. Marini, ${ }^{104}$ M. Marionneau, ${ }^{104}$ P. Martinez Ruiz del Arbol,${ }^{104}$ M. Masciovecchio, ${ }^{104}$ D. Meister, ${ }^{104}$ N. Mohr,${ }^{104}$ P. Musella, ${ }^{104}$ C. Nägeli, ${ }^{104, k k}$ F. Nessi-Tedaldi,${ }^{104}$ F. Pandolfi, ${ }^{104}$ F. Pauss, ${ }^{104}$ L. Perrozzi, ${ }^{104}$ M. Peruzzi, ${ }^{104}$ M. Quittnat, ${ }^{104}$ L. Rebane, ${ }^{104}$ M. Rossini, ${ }^{104}$ A. Starodumov, ${ }^{104,11}$ M. Takahashi, ${ }^{104}$ K. Theofilatos, ${ }^{104}$ R. Wallny, ${ }^{104}$ H. A. Weber, ${ }^{104}$ C. Amsler, ${ }^{105, m m}$ M. F. Canelli, ${ }^{105}$ V. Chiochia, ${ }^{105}$ A. De Cosa, ${ }^{105}$ A. Hinzmann,${ }^{105}$ T. Hreus, ${ }^{105}$ B. Kilminster, ${ }^{105}$ C. Lange, ${ }^{105}$ J. Ngadiuba, ${ }^{105}$ D. Pinna, ${ }^{105}$ P. Robmann, ${ }^{105}$ F. J. Ronga, ${ }^{105}$ S. Taroni, ${ }^{105}$ Y. Yang, ${ }^{105}$ M. Cardaci, ${ }^{106}$ K. H. Chen, ${ }^{106}$ C. Ferro, ${ }^{106}$ C. M. Kuo, ${ }^{106}$ W. Lin,${ }^{106}$ Y. J. Lu ${ }^{106}$ R. Volpe, ${ }^{106}$ S. S. Yu, ${ }^{106}$ P. Chang, ${ }^{107}$ Y. H. Chang, ${ }^{107}$ Y. Chao,${ }^{107}$ K. F. Chen, ${ }^{107}$ P. H. Chen,${ }^{107}$ C. Dietz, ${ }^{107}$ U. Grundler, ${ }^{107}$ W.-S. Hou, ${ }^{107}$ Y. F. Liu, ${ }^{107}$ R.-S. Lu, ${ }^{107}$ M. Miñano Moya, ${ }^{107}$ E. Petrakou, ${ }^{107}$ Y. M. Tzeng,,${ }^{107}$ R. Wilken, ${ }^{107}$ B. Asavapibhop, ${ }^{108}$ G. Singh, ${ }^{108}$ N. Srimanobhas, ${ }^{108}$

N. Suwonjandee, ${ }^{108}$ A. Adiguzel, ${ }^{109}$ M. N. Bakirci, ${ }^{109, n n}$ S. Cerci, ${ }^{109,00}$ C. Dozen, ${ }^{109}$ I. Dumanoglu, ${ }^{109}$ E. Eskut,${ }^{109}$

S. Girgis, ${ }^{109}$ G. Gokbulut, ${ }^{109}$ Y. Guler, ${ }^{109}$ E. Gurpinar, ${ }^{109}$ I. Hos, ${ }^{109}$ E. E. Kangal, ${ }^{109, p p}$ A. Kayis Topaksu, ${ }^{109}$

G. Onengut, ${ }^{109, \mathrm{qq}}$ K. Ozdemir, ${ }^{109, \text { rr }}$ S. Ozturk, ${ }^{109, \text { nn }}$ A. Polatoz, ${ }^{109}$ D. Sunar Cerci, ${ }^{109, o 0}$ B. Tali, ${ }^{109, \text { oo }}$ H. Topakli, ${ }^{109, \text { nn }}$ M. Vergili, ${ }^{109}$ C. Zorbilmez, ${ }^{109}$ I. V. Akin, ${ }^{110}$ B. Bilin, ${ }^{110}$ S. Bilmis, ${ }^{110}$ H. Gamsizkan, ${ }^{110, \text { ss }}$ B. Isildak,,${ }^{110, t t}$ G. Karapinar, ${ }^{110 \text {,u }}$ K. Ocalan, ${ }^{11, \text { vv }}$ S. Sekmen, ${ }^{110}$ U. E. Surat, ${ }^{110}$ M. Yalvac, ${ }^{110}$ M. Zeyrek, ${ }^{110}$ E. A. Albayrak, ${ }^{111, w w}$ E. Gülmez, ${ }^{111}$ M. Kaya, ${ }^{11, \mathrm{xx}}$ O. Kaya, ${ }^{11, y y}$ T. Yetkin, ${ }^{111, z z}$ K. Cankocak, ${ }^{112}$ F. I. Vardarl,,${ }^{112}$ L. Levchuk, ${ }^{113}$ P. Sorokin, ${ }^{113}$ J. J. Brooke, ${ }^{114}$ E. Clement, ${ }^{114}$ D. Cussans, ${ }^{114}$ H. Flacher, ${ }^{114}$ J. Goldstein, ${ }^{114}$ M. Grimes, ${ }^{114}$ G. P. Heath, ${ }^{114}$ H. F. Heath, ${ }^{114}$ J. Jacob, ${ }^{114}$ L. Kreczko, ${ }^{114}$ C. Lucas, ${ }^{114}$ Z. Meng, ${ }^{114}$ D. M. Newbold, ${ }^{114, \text { aaa }}$ S. Paramesvaran, ${ }^{114}$ A. Poll, ${ }^{114}$ T. Sakuma, ${ }^{114}$

S. Seif El Nasr-storey, ${ }^{114}$ S. Senkin, ${ }^{114}$ V. J. Smith, ${ }^{114}$ K. W. Bell, ${ }^{115}$ A. Belyaev, ${ }^{115, b b b}$ C. Brew, ${ }^{115}$ R. M. Brown, ${ }^{115}$ D. J. A. Cockerill, ${ }^{115}$ J. A. Coughlan, ${ }^{115}$ K. Harder, ${ }^{115}$ S. Harper, ${ }^{115}$ E. Olaiya, ${ }^{115}$ D. Petyt,,${ }^{115}$

C. H. Shepherd-Themistocleous, ${ }^{115}$ A. Thea, ${ }^{115}$ I. R. Tomalin, ${ }^{115}$ T. Williams, ${ }^{115}$ W. J. Womersley, ${ }^{115}$ S. D. Worm, ${ }^{115}$ M. Baber, ${ }^{116}$ R. Bainbridge, ${ }^{116}$ O. Buchmuller, ${ }^{116}$ D. Burton, ${ }^{116}$ D. Colling, ${ }^{116}$ N. Cripps, ${ }^{116}$ P. Dauncey, ${ }^{116}$ G. Davies, ${ }^{116}$ M. Della Negra, ${ }^{116}$ P. Dunne, ${ }^{116}$ A. Elwood, ${ }^{116}$ W. Ferguson, ${ }^{116}$ J. Fulcher, ${ }^{116}$ D. Futyan, ${ }^{116}$ G. Hall, ${ }^{116}$ G. Iles, ${ }^{116}$ M. Jarvis, ${ }^{116}$ G. Karapostoli, ${ }^{116}$ M. Kenzie, ${ }^{116}$ R. Lane, ${ }^{116}$ R. Lucas, ${ }^{116 \text {,aa }}$ L. Lyons, ${ }^{116}$ A.-M. Magnan, ${ }^{116}$ S. Malik, ${ }^{116}$ B. Mathias, ${ }^{116}$ J. Nash, ${ }^{116}$ A. Nikitenko, ${ }^{116,11}$ J. Pela,${ }^{116}$ M. Pesaresi,${ }^{116}$ K. Petridis, ${ }^{116}$ D. M. Raymond,${ }^{116}$ S. Rogerson, ${ }^{116}$ A. Rose, ${ }^{116}$ C. Seez, ${ }^{116}$ P. Sharp, ${ }^{116, a}$ A. Tapper,${ }^{116}$ M. Vazquez Acosta, ${ }^{116}$ T. Virdee, ${ }^{116}$ S. C. Zenz, ${ }^{116}$ J. E. Cole, ${ }^{117}$ P. R. Hobson, ${ }^{117}$ A. Khan, ${ }^{117}$ P. Kyberd, ${ }^{117}$ D. Leggat, ${ }^{117}$ D. Leslie, ${ }^{117}$ I. D. Reid, ${ }^{117}$ P. Symonds, ${ }^{117}$ L. Teodorescu, ${ }^{117}$ M. Turner, ${ }^{117}$ J. Dittmann, ${ }^{118}$ K. Hatakeyama, ${ }^{118}$ A. Kasmi, ${ }^{118}$ H. Liu, ${ }^{118}$ N. Pastika, ${ }^{118}$ T. Scarborough, ${ }^{118}$ Z. Wu, ${ }^{118}$ O. Charaf, ${ }^{119}$ S. I. Cooper, ${ }^{119}$ C. Henderson, ${ }^{119}$ P. Rumerio, ${ }^{119}$ A. Avetisyan, ${ }^{120}$ T. Bose, ${ }^{120}$ C. Fantasia, ${ }^{120}$ P. Lawson, ${ }^{120}$ C. Richardson, ${ }^{120}$ J. Rohlf,${ }^{120}$ J. St. John, ${ }^{120}$ L. Sulak, ${ }^{120}$ J. Alimena, ${ }^{121}$ E. Berry, ${ }^{121}$ S. Bhattacharya, ${ }^{121}$ G. Christopher, ${ }^{121}$ D. Cutts, ${ }^{121}$ Z. Demiragli, ${ }^{121}$ N. Dhingra, ${ }^{121}$ A. Ferapontov, ${ }^{121}$ A. Garabedian, ${ }^{121}$ U. Heintz,${ }^{121}$ G. Kukartsev, ${ }^{121}$ E. Laird, ${ }^{121}$ G. Landsberg, ${ }^{121}$ M. Luk, ${ }^{121}$ M. Narain, ${ }^{121}$ M. Segala, ${ }^{121}$ T. Sinthuprasith, ${ }^{121}$ T. Speer, ${ }^{121}$ J. Swanson, ${ }^{121}$ R. Breedon, ${ }^{122}$ G. Breto, ${ }^{122}$ M. Calderon De La Barca Sanchez, ${ }^{122}$ S. Chauhan, ${ }^{122}$ M. Chertok, ${ }^{122}$ J. Conway, ${ }^{122}$ R. Conway, ${ }^{122}$ P. T. Cox, ${ }^{122}$ R. Erbacher ${ }^{122}$ M. Gardner, ${ }^{122}$ W. Ko, ${ }^{122}$ R. Lander, ${ }^{122}$ M. Mulhearn, ${ }^{122}$ D. Pellett, ${ }^{122}$ J. Pilot, ${ }^{122}$ F. Ricci-Tam, ${ }^{122}$ S. Shalhout, ${ }^{122}$ J. Smith, ${ }^{122}$ M. Squires, ${ }^{122}$ D. Stolp,${ }^{122}$ M. Tripathi, ${ }^{122}$ S. Wilbur, ${ }^{122}$ R. Yohay, ${ }^{122}$ R. Cousins, ${ }^{123}$ P. Everaerts, ${ }^{123}$ C. Farrell, ${ }^{123}$ J. Hauser, ${ }^{123}$ M. Ignatenko, ${ }^{123}$ G. Rakness, ${ }^{123}$ E. Takasugi, ${ }^{123}$ V. Valuev, ${ }^{123}$ M. Weber, ${ }^{123}$ K. Burt, ${ }^{124}$ R. Clare, ${ }^{124}$ J. Ellison, ${ }^{124}$ J. W. Gary, ${ }^{124}$ G. Hanson, ${ }^{124}$ J. Heilman, ${ }^{124}$ M. Ivova Rikova, ${ }^{124}$ P. Jandir, ${ }^{124}$ E. Kennedy, ${ }^{124}$ F. Lacroix, ${ }^{124}$ O. R. Long, ${ }^{124}$ A. Luthra, ${ }^{124}$ M. Malberti, ${ }^{124}$ M. Olmedo Negrete, ${ }^{124}$ A. Shrinivas, ${ }^{124}$ S. Sumowidagdo, ${ }^{124}$ S. Wimpenny, ${ }^{124}$ J. G. Branson, ${ }^{125}$ G. B. Cerati, ${ }^{125}$ S. Cittolin, ${ }^{125}$ R. T. D'Agnolo, ${ }^{125}$ A. Holzner, ${ }^{125}$ R. Kelley, ${ }^{125}$ D. Klein, ${ }^{125}$ J. Letts, ${ }^{125}$ I. Macneill, ${ }^{125}$ D. Olivito, ${ }^{125}$ S. Padhi, ${ }^{125}$ C. Palmer, ${ }^{125}$ M. Pieri, ${ }^{125}$ M. Sani, ${ }^{125}$ 
V. Sharma, ${ }^{125}$ S. Simon, ${ }^{125}$ M. Tadel, ${ }^{125}$ Y. Tu, ${ }^{125}$ A. Vartak, ${ }^{125}$ C. Welke, ${ }^{125}$ F. Würthwein, ${ }^{125}$ A. Yagil, ${ }^{125}$ G. Zevi Della Porta, ${ }^{125}$ D. Barge, ${ }^{126}$ J. Bradmiller-Feld, ${ }^{126}$ C. Campagnari, ${ }^{126}$ T. Danielson, ${ }^{126}$ A. Dishaw, ${ }^{126}$ V. Dutta, ${ }^{126}$ K. Flowers, ${ }^{126}$ M. Franco Sevilla, ${ }^{126}$ P. Geffert, ${ }^{126}$ C. George, ${ }^{126}$ F. Golf, ${ }^{126}$ L. Gouskos, ${ }^{126}$ J. Incandela, ${ }^{126}$ C. Justus, ${ }^{126}$ N. Mccoll, ${ }^{126}$ S. D. Mullin, ${ }^{126}$ J. Richman, ${ }^{126}$ D. Stuart, ${ }^{126}$ W. To, ${ }^{126}$ C. West,,${ }^{126}$ J. Yoo, ${ }^{126}$ A. Apresyan, ${ }^{127}$ A. Bornheim, ${ }^{127}$ J. Bunn, ${ }^{127}$ Y. Chen, ${ }^{127}$ J. Duarte, ${ }^{127}$ A. Mott, ${ }^{127}$ H. B. Newman, ${ }^{127}$ C. Pena, ${ }^{127}$ M. Pierini, ${ }^{127}$ M. Spiropulu, ${ }^{127}$ J. R. Vlimant, ${ }^{127}$ R. Wilkinson, ${ }^{127}$ S. Xie, ${ }^{127}$ R. Y. Zhu, ${ }^{127}$ V. Azzolini, ${ }^{128}$ A. Calamba, ${ }^{128}$ B. Carlson, ${ }^{128}$ T. Ferguson, ${ }^{128}$ Y. Iiyama, ${ }^{128}$ M. Paulini, ${ }^{128}$ J. Russ, ${ }^{128}$ H. Vogel, ${ }^{128}$ I. Vorobiev, ${ }^{128}$ J. P. Cumalat, ${ }^{129}$ W. T. Ford, ${ }^{129}$ A. Gaz, ${ }^{129}$ M. Krohn, ${ }^{129}$ E. Luiggi Lopez, ${ }^{129}$ U. Nauenberg, ${ }^{129}$ J. G. Smith, ${ }^{129}$ K. Stenson, ${ }^{129}$ S. R. Wagner, ${ }^{129}$ J. Alexander, ${ }^{130}$ A. Chatterjee, ${ }^{130}$ J. Chaves, ${ }^{130}$ J. Chu, ${ }^{130}$ S. Dittmer, ${ }^{130}$ N. Eggert, ${ }^{130}$ N. Mirman, ${ }^{130}$ G. Nicolas Kaufman, ${ }^{130}$ J. R. Patterson, ${ }^{130}$ A. Ryd, ${ }^{130}$ E. Salvati, ${ }^{130}$ L. Skinnari, ${ }^{130}$ W. Sun, ${ }^{130}$ W. D. Teo, ${ }^{130}$ J. Thom, ${ }^{130}$ J. Thompson, ${ }^{130}$ J. Tucker, ${ }^{130}$ Y. Weng, ${ }^{130}$ L. Winstrom, ${ }^{130}$ P. Wittich ${ }^{130}$ D. Winn, ${ }^{131}$ S. Abdullin, ${ }^{132}$ M. Albrow, ${ }^{132}$ J. Anderson, ${ }^{132}$ G. Apollinari, ${ }^{132}$ L. A. T. Bauerdick, ${ }^{132}$ A. Beretvas, ${ }^{132}$ J. Berryhill, ${ }^{132}$ P. C. Bhat, ${ }^{132}$ G. Bolla, ${ }^{132}$ K. Burkett, ${ }^{132}$ J. N. Butler, ${ }^{132}$ H. W. K. Cheung, ${ }^{132}$ F. Chlebana, ${ }^{132}$ S. Cihangir, ${ }^{132}$ V. D. Elvira, ${ }^{132}$ I. Fisk, ${ }^{132}$ J. Freeman, ${ }^{132}$ E. Gottschalk, ${ }^{132}$ L. Gray, ${ }^{132}$ D. Green, ${ }^{132}$ S. Grünendahl, ${ }^{132}$ O. Gutsche, ${ }^{132}$ J. Hanlon, ${ }^{132}$ D. Hare, ${ }^{132}$ R. M. Harris, ${ }^{132}$ J. Hirschauer, ${ }^{132}$ B. Hooberman, ${ }^{132}$ S. Jindariani, ${ }^{132}$ M. Johnson, ${ }^{132}$ U. Joshi, ${ }^{132}$ B. Klima, ${ }^{132}$ B. Kreis, ${ }^{132}$ S. Kwan, ${ }^{132, a}$ J. Linacre, ${ }^{132}$ D. Lincoln, ${ }^{132}$ R. Lipton, ${ }^{132}$ T. Liu, ${ }^{132}$ J. Lykken, ${ }^{132}$ K. Maeshima, ${ }^{132}$ J. M. Marraffino, ${ }^{132}$ V. I. Martinez Outschoorn, ${ }^{132}$ S. Maruyama, ${ }^{132}$ D. Mason, ${ }^{132}$ P. McBride, ${ }^{132}$ P. Merkel, ${ }^{132}$ K. Mishra, ${ }^{132}$ S. Mrenna, ${ }^{132}$ S. Nahn, ${ }^{132}$ C. Newman-Holmes, ${ }^{132}$ V. O'Dell, ${ }^{132}$ O. Prokofyev, ${ }^{132}$ E. Sexton-Kennedy, ${ }^{132}$ A. Soha, ${ }^{132}$ W. J. Spalding, ${ }^{132}$ L. Spiegel, ${ }^{132}$ L. Taylor, ${ }^{132}$ S. Tkaczyk, ${ }^{132}$ N. V. Tran, ${ }^{132}$ L. Uplegger, ${ }^{132}$ E. W. Vaandering, ${ }^{132}$ R. Vidal, ${ }^{132}$ A. Whitbeck,${ }^{132}$ J. Whitmore, ${ }^{132}$ F. Yang, ${ }^{132}$ D. Acosta, ${ }^{133}$ P. Avery, ${ }^{133}$ P. Bortignon, ${ }^{133}$ D. Bourilkov, ${ }^{133}$ M. Carver, ${ }^{133}$ D. Curry, ${ }^{133}$ S. Das, ${ }^{133}$ M. De Gruttola, ${ }^{133}$ G. P. Di Giovanni, ${ }^{133}$ R. D. Field, ${ }^{133}$ M. Fisher, ${ }^{133}$ I. K. Furic, ${ }^{133}$ J. Hugon, ${ }^{133}$ J. Konigsberg, ${ }^{133}$ A. Korytov, ${ }^{133}$ T. Kypreos, ${ }^{133}$ J. F. Low, ${ }^{133}$ K. Matchev, ${ }^{133}$ H. Mei, ${ }^{133}$ P. Milenovic, ${ }^{133, c c c}$ G. Mitselmakher, ${ }^{133}$ L. Muniz, ${ }^{133}$ A. Rinkevicius, ${ }^{133}$ L. Shchutska, ${ }^{133}$ M. Snowball, ${ }^{133}$ D. Sperka, ${ }^{133}$ J. Yelton, ${ }^{133}$ M. Zakaria, ${ }^{133}$ S. Hewamanage, ${ }^{134}$ S. Linn, ${ }^{134}$ P. Markowitz,${ }^{134}$ G. Martinez, ${ }^{134}$ J. L. Rodriguez, ${ }^{134}$ J. R. Adams, ${ }^{135}$ T. Adams, ${ }^{135}$ A. Askew, ${ }^{135}$ J. Bochenek, ${ }^{135}$ B. Diamond, ${ }^{135}$ J. Haas, ${ }^{135}$ S. Hagopian, ${ }^{135}$ V. Hagopian, ${ }^{135}$ K. F. Johnson, ${ }^{135}$ H. Prosper ${ }^{135}$ V. Veeraraghavan, ${ }^{135}$ M. Weinberg, ${ }^{135}$ M. M. Baarmand, ${ }^{136}$

M. Hohlmann, ${ }^{136}$ H. Kalakhety, ${ }^{136}$ F. Yumiceva, ${ }^{136}$ M. R. Adams, ${ }^{137}$ L. Apanasevich ${ }^{137}$ D. Berry, ${ }^{137}$ R. R. Betts, ${ }^{137}$ I. Bucinskaite, ${ }^{137}$ R. Cavanaugh, ${ }^{137}$ O. Evdokimov, ${ }^{137}$ L. Gauthier, ${ }^{137}$ C. E. Gerber, ${ }^{137}$ D. J. Hofman, ${ }^{137}$ P. Kurt, ${ }^{137}$ C. O’Brien, ${ }^{137}$ I. D. Sandoval Gonzalez, ${ }^{137}$ C. Silkworth,${ }^{137}$ P. Turner,${ }^{137}$ N. Varelas, ${ }^{137}$ B. Bilki, ${ }^{138, d d d}$ W. Clarida, ${ }^{138}$ K. Dilsiz, ${ }^{138}$ M. Haytmyradov, ${ }^{138}$ J.-P. Merlo, ${ }^{138}$ H. Mermerkaya,${ }^{138, \text { eee }}$ A. Mestvirishvili, ${ }^{138}$ A. Moeller, ${ }^{138}$ J. Nachtman, ${ }^{138}$ H. Ogul, ${ }^{138}$ Y. Onel, ${ }^{138}$ F. Ozok, ${ }^{138, w w}$ A. Penzo, ${ }^{138}$ R. Rahmat, ${ }^{138}$ S. Sen, ${ }^{138}$ P. Tan, ${ }^{138}$ E. Tiras, ${ }^{138}$ J. Wetzel, ${ }^{138}$ K. Yi, ${ }^{138}$ I. Anderson, ${ }^{139}$ B. A. Barnett, ${ }^{139}$ B. Blumenfeld, ${ }^{139}$ S. Bolognesi, ${ }^{139}$ D. Fehling, ${ }^{139}$ A. V. Gritsan, ${ }^{139}$ P. Maksimovic, ${ }^{139}$ C. Martin, ${ }^{139}$ M. Swartz, ${ }^{139}$ M. Xiao, ${ }^{139}$ P. Baringer, ${ }^{140}$ A. Bean, ${ }^{140}$ G. Benelli, ${ }^{140}$ C. Bruner, ${ }^{140}$ J. Gray, ${ }^{140}$ R. P. Kenny III, ${ }^{140}$ D. Majumder, ${ }^{140}$ M. Malek, ${ }^{140}$ M. Murray, ${ }^{140}$ D. Noonan, ${ }^{140}$ S. Sanders, ${ }^{140}$ J. Sekaric, ${ }^{140}$ R. Stringer, ${ }^{140}$ Q. Wang, ${ }^{140}$ J. S. Wood, ${ }^{140}$ I. Chakaberia, ${ }^{141}$ A. Ivanov, ${ }^{141}$ K. Kaadze, ${ }^{141}$ S. Khalil, ${ }^{141}$ M. Makouski, ${ }^{141}$ Y. Maravin, ${ }^{141}$ L. K. Saini, ${ }^{141}$ N. Skhirtladze ${ }^{141}$ I. Svintradze, ${ }^{141}$ J. Gronberg, ${ }^{142}$ D. Lange,${ }^{142}$ F. Rebassoo,${ }^{142}$ D. Wright, ${ }^{142}$ A. Baden, ${ }^{143}$ A. Belloni, ${ }^{143}$

B. Calvert, ${ }^{143}$ S. C. Eno, ${ }^{143}$ J. A. Gomez, ${ }^{143}$ N. J. Hadley, ${ }^{143}$ S. Jabeen, ${ }^{143}$ R. G. Kellogg, ${ }^{143}$ T. Kolberg, ${ }^{143}$ Y. Lu, ${ }^{143}$ A. C. Mignerey, ${ }^{143}$ K. Pedro, ${ }^{143}$ A. Skuja, ${ }^{143}$ M. B. Tonjes, ${ }^{143}$ S. C. Tonwar, ${ }^{143}$ A. Apyan, ${ }^{144}$ R. Barbieri, ${ }^{144}$ K. Bierwagen, ${ }^{144}$ W. Busza, ${ }^{144}$ I. A. Cali, ${ }^{144}$ L. Di Matteo, ${ }^{144}$ G. Gomez Ceballos, ${ }^{144}$ M. Goncharov, ${ }^{144}$ D. Gulhan, ${ }^{144}$ M. Klute,${ }^{144}$ Y. S. Lai, ${ }^{144}$ Y.-J. Lee, ${ }^{144}$ A. Levin, ${ }^{144}$ P. D. Luckey, ${ }^{144}$ C. Paus, ${ }^{144}$ D. Ralph,${ }^{144}$ C. Roland,${ }^{144}$ G. Roland, ${ }^{144}$ G. S. F. Stephans, ${ }^{144}$ K. Sumorok, ${ }^{144}$ D. Velicanu, ${ }^{144}$ J. Veverka ${ }^{144}$ B. Wyslouch,${ }^{144}$ M. Yang, ${ }^{144}$ M. Zanetti ${ }^{144}$ V. Zhukova ${ }^{144}$ B. Dahmes, ${ }^{145}$ A. Gude ${ }^{145}$ S. C. Kao, ${ }^{145}$ K. Klapoetke, ${ }^{145}$ Y. Kubota, ${ }^{145}$ J. Mans, ${ }^{145}$ S. Nourbakhsh, ${ }^{145}$ R. Rusack, ${ }^{145}$ A. Singovsky, ${ }^{145}$ N. Tambe, ${ }^{145}$ J. Turkewitz, ${ }^{145}$ J. G. Acosta, ${ }^{146}$ S. Oliveros, ${ }^{146}$ E. Avdeeva, ${ }^{147}$ K. Bloom, ${ }^{147}$ S. Bose,${ }^{147}$ D. R. Claes, ${ }^{147}$ A. Dominguez ${ }^{147}$ R. Gonzalez Suarez, ${ }^{147}$ J. Keller, ${ }^{147}$ D. Knowlton, ${ }^{147}$ I. Kravchenko, ${ }^{147}$ J. Lazo-Flores,${ }^{147}$ F. Meier, ${ }^{147}$ F. Ratnikov, ${ }^{147}$ G. R. Snow, ${ }^{147}$ M. Zvada, ${ }^{147}$ J. Dolen, ${ }^{148}$ A. Godshalk, ${ }^{148}$ I. Iashvilit ${ }^{148}$ A. Kharchilava, ${ }^{148}$ A. Kumar, ${ }^{148}$ S. Rappoccio, ${ }^{148}$ G. Alverson, ${ }^{149}$ E. Barberis,${ }^{149}$ D. Baumgartel,${ }^{149}$ M. Chasco, ${ }^{149}$ A. Massironi, ${ }^{149}$ D. M. Morse,${ }^{149}$ D. Nash, ${ }^{149}$ T. Orimoto, ${ }^{149}$ D. Trocino, ${ }^{149}$ R.-J. Wang, ${ }^{149}$ D. Wood,,${ }^{149}$ J. Zhang, ${ }^{149}$ K. A. Hahn, ${ }^{150}$ A. Kubik,,${ }^{150}$ N. Mucia, ${ }^{150}$ N. Odell, ${ }^{150}$ B. Pollack, ${ }^{150}$ A. Pozdnyakov, ${ }^{150}$ M. Schmitt, ${ }^{150}$ S. Stoynev, ${ }^{150}$ K. Sung, ${ }^{150}$ M. Velasco, ${ }^{150}$ S. Won, ${ }^{150}$ A. Brinkerhoff, ${ }^{151}$ K. M. Chan, ${ }^{151}$ A. Drozdetskiy, ${ }^{151}$ M. Hildreth, ${ }^{151}$ C. Jessop,${ }^{151}$ D. J. Karmgard, ${ }^{151}$ N. Kellams, ${ }^{151}$ K. Lannon, ${ }^{151}$ S. Lynch, ${ }^{151}$ N. Marinelli, ${ }^{151}$ Y. Musienko, ${ }^{151, d d}$ T. Pearson, ${ }^{151}$ M. Planer, ${ }^{151}$ R. Ruchti, ${ }^{151}$ G. Smith, ${ }^{151}$ 
N. Valls ${ }_{151}^{151}$ M. Wayne ${ }^{151}$ M. Wolf, ${ }^{151}$ A. Woodard,${ }^{151}$ L. Antonelli, ${ }^{152}$ J. Brinson, ${ }^{152}$ B. Bylsma, ${ }^{152}$ L. S. Durkin, ${ }^{152}$ S. Flowers, ${ }^{152}$ A. Hart,${ }^{152}$ C. Hill,${ }^{152}$ R. Hughes, ${ }^{152}$ K. Kotov,${ }^{152}$ T. Y. Ling, ${ }^{152}$ W. Luo, ${ }^{152}$ D. Puigh,${ }^{152}$ M. Rodenburg, ${ }^{152}$ B. L. Winer, ${ }^{152}$ H. Wolfe, ${ }^{152}$ H. W. Wulsin, ${ }^{152}$ O. Driga,${ }^{153}$ P. Elmer, ${ }^{153}$ J. Hardenbrook, ${ }^{153}$ P. Hebda, ${ }^{153}$ S. A. Koay, ${ }^{153}$ P. Lujan, ${ }^{153}$ D. Marlow, ${ }^{153}$ T. Medvedeva, ${ }^{153}$ M. Mooney, ${ }^{153}$ J. Olsen, ${ }^{153}$ P. Piroué ${ }^{153}$ X. Quan, ${ }^{153}$ H. Saka ${ }^{153}$ D. Stickland, ${ }^{153, c}$ C. Tully, ${ }^{153}$ J. S. Werner, ${ }^{153}$ A. Zuranski, ${ }^{153}$ E. Brownson, ${ }^{154}$ S. Malik, ${ }^{154}$ H. Mendez, ${ }^{154}$ J.E. Ramirez Vargas, ${ }^{154}$ V. E. Barnes, ${ }^{155}$ D. Benedetti, ${ }^{155}$ D. Bortoletto, ${ }^{155}$ M. De Mattia,${ }^{155}$ L. Gutay, ${ }^{155}$ Z. Hu, ${ }^{155}$ M. K. Jha, ${ }^{155}$ M. Jones, ${ }^{155}$ K. Jung ${ }^{155}$ M. Kress, ${ }^{155}$ N. Leonardo, ${ }^{155}$ D. H. Miller, ${ }^{155}$ N. Neumeister, ${ }^{155}$ F. Primavera, ${ }^{155}$ B. C. Radburn-Smith, ${ }^{155}$ X. Shi, ${ }^{155}$ I. Shipsey, ${ }^{155}$ D. Silvers, ${ }^{155}$ A. Svyatkovskiy, ${ }^{155}$ F. Wang, ${ }^{155}$ W. Xie, ${ }^{155}$ L. Xu, ${ }^{155}$ J. Zablocki, ${ }^{155}$ N. Parashar, ${ }^{156}$ J. Stupak, ${ }^{156}$ A. Adair, ${ }^{157}$ B. Akgun, ${ }^{157}$ K. M. Ecklund, ${ }^{157}$ F. J. M. Geurts, ${ }^{157}$ W. Li, ${ }^{157}$ B. Michlin, ${ }^{157}$ B. P. Padley, ${ }^{157}$ R. Redjimi, ${ }^{157}$ J. Roberts, ${ }^{157}$ J. Zabel, ${ }^{157}$ B. Betchart, ${ }^{158}$ A. Bodek, ${ }^{158}$ P. de Barbaro, ${ }^{158}$ R. Demina, ${ }^{158}$ Y. Eshaq, ${ }^{158}$ T. Ferbel, ${ }^{158}$ M. Galanti, ${ }^{158}$ A. Garcia-Bellido, ${ }^{158}$ P. Goldenzweig, ${ }^{158}$ J. Han, ${ }^{158}$ A. Harel, ${ }^{158}$ O. Hindrichs, ${ }^{158}$ A. Khukhunaishvili, ${ }^{158}$ S. Korjenevski, ${ }^{158}$ G. Petrillo, ${ }^{158}$ M. Verzetti, ${ }^{158}$ D. Vishnevskiy, ${ }^{158}$ R. Ciesielski, ${ }^{159}$ L. Demortier, ${ }^{159}$ K. Goulianos, ${ }^{159}$ C. Mesropian, ${ }^{159}$ S. Arora,${ }^{160}$ A. Barker,${ }^{160}$ J. P. Chou, ${ }^{160}$ C. Contreras-Campana, ${ }^{160}$ E. Contreras-Campana, ${ }^{160}$ D. Duggan, ${ }^{160}$ D. Ferencek, ${ }^{160}$ Y. Gershtein, ${ }^{160}$ R. Gray, ${ }^{160}$ E. Halkiadakis, ${ }^{160}$ D. Hidas, ${ }^{160}$ S. Kaplan, ${ }^{160}$ A. Lath, ${ }^{160}$ S. Panwalkar, ${ }^{160}$ M. Park, ${ }^{160}$ R. Patel, ${ }^{160}$ S. Salur, ${ }^{160}$ S. Schnetzer, ${ }^{160}$ D. Sheffield, ${ }^{160}$ S. Somalwar, ${ }^{160}$ R. Stone, ${ }^{160}$ S. Thomas, ${ }^{160}$ P. Thomassen, ${ }^{160}$ M. Walker, ${ }^{160}$ K. Rose, ${ }^{161}$ S. Spanier, ${ }^{161}$ A. York, ${ }^{161}$ O. Bouhali, ${ }^{162, f f f}$ A. Castaneda Hernandez, ${ }^{162}$ R. Eusebi, ${ }^{162}$ W. Flanagan, ${ }^{162}$ J. Gilmore, ${ }^{162}$ T. Kamon, ${ }^{162, g g g}$ V. Khotilovich, ${ }^{162}$ V. Krutelyov, ${ }^{162}$ R. Montalvo, ${ }^{162}$ I. Osipenkov, ${ }^{162}$ Y. Pakhotin, ${ }^{162}$ A. Perloff, ${ }^{162}$ J. Roe, ${ }^{162}$ A. Rose, ${ }^{162}$ A. Safonov, ${ }^{162}$ I. Suarez, ${ }^{162}$ A. Tatarinov, ${ }^{162}$ K. A. Ulmer, ${ }^{162}$ N. Akchurin, ${ }^{163}$ C. Cowden, ${ }^{163}$ J. Damgov, ${ }^{163}$ C. Dragoiu, ${ }^{163}$ P. R. Dudero, ${ }^{163}$ J. Faulkner, ${ }^{163}$ K. Kovitanggoon, ${ }^{163}$ S. Kunori, ${ }^{163}$ S. W. Lee, ${ }^{163}$ T. Libeiro, ${ }^{163}$ I. Volobouev, ${ }^{163}$ E. Appelt, ${ }^{164}$ A. G. Delannoy, ${ }^{164}$ S. Greene, ${ }^{164}$ A. Gurrola, ${ }^{164}$ W. Johns, ${ }^{164}$ C. Maguire ${ }^{164}$ Y. Mao, ${ }^{164}$ A. Melo, ${ }^{164}$ M. Sharma, ${ }^{164}$ P. Sheldon, ${ }^{164}$ B. Snook, ${ }^{164}$ S. Tuo, ${ }^{164}$ J. Velkovska, ${ }^{164}$ M. W. Arenton, ${ }^{165}$ S. Boutle, ${ }^{165}$ B. Cox,${ }^{165}$ B. Francis, ${ }^{165}$ J. Goodell, ${ }^{165}$ R. Hirosky, ${ }^{165}$ A. Ledovskoy, ${ }^{165}$ H. Li ${ }^{165}$ C. Lin,${ }^{165}$ C. Neu, ${ }^{165}$ E. Wolfe, ${ }^{165}$ J. Wood, ${ }^{165}$ C. Clarke, ${ }^{166}$ R. Harr, ${ }^{166}$ P. E. Karchin, ${ }^{166}$ C. Kottachchi Kankanamge Don, ${ }^{166}$ P. Lamichhane, ${ }^{166}$ J. Sturdy, ${ }^{166}$ D. A. Belknap, ${ }^{167}$ D. Carlsmith, ${ }^{167}$ M. Cepeda, ${ }^{167}$ S. Dasu, ${ }^{167}$ L. Dodd, ${ }^{167}$ S. Duric, ${ }^{167}$ E. Friis, ${ }^{167}$ R. Hall-Wilton, ${ }^{167}$ M. Herndon, ${ }^{167}$ A. Hervé, ${ }^{167}$ P. Klabbers, ${ }^{167}$ A. Lanaro, ${ }^{167}$ C. Lazaridis, ${ }^{167}$ A. Levine, ${ }^{167}$ R. Loveless, ${ }^{167}$ A. Mohapatra, ${ }^{167}$ I. Ojalvo, ${ }^{167}$ T. Perry, ${ }^{167}$ G. A. Pierro, ${ }^{167}$ G. Polese,${ }^{167}$ I. Ross,${ }^{167}$ T. Sarangi, ${ }^{167}$ A. Savin, ${ }^{167}$ W. H. Smith, ${ }^{167}$ D. Taylor, ${ }^{167}$ C. Vuosalo ${ }^{167}$ and N. Woods ${ }^{167}$

\title{
(CMS Collaboration)
}

\author{
${ }^{1}$ Yerevan Physics Institute, Yerevan, Armenia \\ ${ }^{2}$ Institut für Hochenergiephysik der OeAW, Wien, Austria \\ ${ }^{3}$ National Centre for Particle and High Energy Physics, Minsk, Belarus \\ ${ }^{4}$ Universiteit Antwerpen, Antwerpen, Belgium \\ ${ }^{5}$ Vrije Universiteit Brussel, Brussel, Belgium \\ ${ }^{6}$ Université Libre de Bruxelles, Bruxelles, Belgium \\ ${ }^{7}$ Ghent University, Ghent, Belgium \\ ${ }^{8}$ Université Catholique de Louvain, Louvain-la-Neuve, Belgium \\ ${ }^{9}$ Université de Mons, Mons, Belgium \\ ${ }^{10}$ Centro Brasileiro de Pesquisas Fisicas, Rio de Janeiro, Brazil \\ ${ }^{11}$ Universidade do Estado do Rio de Janeiro, Rio de Janeiro, Brazil \\ ${ }^{12 a}$ Universidade Estadual Paulista, São Paulo, Brazil \\ ${ }^{12 \mathrm{~b}}$ Universidade Federal do ABC, São Paulo, Brazil \\ ${ }^{13}$ Institute for Nuclear Research and Nuclear Energy, Sofia, Bulgaria \\ ${ }^{14}$ University of Sofia, Sofia, Bulgaria \\ ${ }^{15}$ Institute of High Energy Physics, Beijing, China \\ ${ }^{16}$ State Key Laboratory of Nuclear Physics and Technology, Peking University, Beijing, China \\ ${ }^{17}$ Universidad de Los Andes, Bogota, Colombia \\ ${ }^{18}$ University of Split, Faculty of Electrical Engineering, \\ Mechanical Engineering and Naval Architecture, Split, Croatia \\ ${ }^{19}$ University of Split, Faculty of Science, Split, Croatia \\ ${ }^{20}$ Institute Rudjer Boskovic, Zagreb, Croatia
}




\footnotetext{
${ }^{21}$ University of Cyprus, Nicosia, Cyprus

${ }^{22}$ Charles University, Prague, Czech Republic
}

${ }^{23}$ Academy of Scientific Research and Technology of the Arab Republic of Egypt,

Egyptian Network of High Energy Physics, Cairo, Egypt

${ }^{24}$ National Institute of Chemical Physics and Biophysics, Tallinn, Estonia

${ }^{25}$ Department of Physics, University of Helsinki, Helsinki, Finland

${ }^{26}$ Helsinki Institute of Physics, Helsinki, Finland

${ }^{27}$ Lappeenranta University of Technology, Lappeenranta, Finland

${ }^{28}$ DSM/IRFU, CEA/Saclay, Gif-sur-Yvette, France

${ }^{29}$ Laboratoire Leprince-Ringuet, Ecole Polytechnique, IN2P3-CNRS, Palaiseau, France

${ }^{30}$ Institut Pluridisciplinaire Hubert Curien, Université de Strasbourg,

Université de Haute Alsace Mulhouse, CNRS/IN2P3, Strasbourg, France

${ }^{31}$ Centre de Calcul de l'Institut National de Physique Nucleaire et de Physique des Particules,

CNRS/IN2P3, Villeurbanne, France

${ }^{32}$ Université de Lyon, Université Claude Bernard Lyon 1, CNRS-IN2P3,

Institut de Physique Nucléaire de Lyon, Villeurbanne, France

${ }^{33}$ Institute of High Energy Physics and Informatization, Tbilisi State University, Tbilisi, Georgia

${ }^{34}$ RWTH Aachen University, I. Physikalisches Institut, Aachen, Germany

${ }^{35}$ RWTH Aachen University, III. Physikalisches Institut A, Aachen, Germany

${ }^{36}$ RWTH Aachen University, III. Physikalisches Institut B, Aachen, Germany

${ }^{37}$ Deutsches Elektronen-Synchrotron, Hamburg, Germany

${ }^{38}$ University of Hamburg, Hamburg, Germany

${ }^{39}$ Institut für Experimentelle Kernphysik, Karlsruhe, Germany

${ }^{40}$ Institute of Nuclear and Particle Physics (INPP), NCSR Demokritos, Aghia Paraskevi, Greece

${ }^{41}$ University of Athens, Athens, Greece

${ }^{42}$ University of Ioánnina, Ioánnina, Greece

${ }^{43}$ Wigner Research Centre for Physics, Budapest, Hungary

${ }^{44}$ Institute of Nuclear Research ATOMKI, Debrecen, Hungary

${ }^{45}$ University of Debrecen, Debrecen, Hungary

${ }^{46}$ National Institute of Science Education and Research, Bhubaneswar, India

${ }^{47}$ Panjab University, Chandigarh, India

${ }^{48}$ University of Delhi, Delhi, India

${ }^{49}$ Saha Institute of Nuclear Physics, Kolkata, India

${ }^{50}$ Bhabha Atomic Research Centre, Mumbai, India

${ }^{51}$ Tata Institute of Fundamental Research, Mumbai, India

${ }^{52}$ Indian Institute of Science Education and Research (IISER), Pune, India

${ }^{53}$ Institute for Research in Fundamental Sciences (IPM), Tehran, Iran

${ }^{54}$ University College Dublin, Dublin, Ireland

${ }^{55 a}$ INFN Sezione di Bari, Bari, Italy

${ }^{55 \mathrm{~b}}$ Università di Bari, Bari, Italy

${ }^{55 \mathrm{c}}$ Politecnico di Bari, Bari, Italy

${ }^{56 a}$ INFN Sezione di Bologna, Bologna, Italy

${ }^{56 \mathrm{~b}}$ Università di Bologna, Bologna, Italy

${ }^{57}$ INFN Sezione di Catania, Catania, Italy

${ }^{57 \mathrm{~b}}$ Università di Catania, Catania, Italy

${ }^{57 \mathrm{c} C S F N S M, \text { Catania, Italy }}$

${ }^{58 \mathrm{a}}$ INFN Sezione di Firenze, Firenze, Italy

${ }^{58 \mathrm{~b}}$ Università di Firenze, Firenze, Italy

${ }^{59}$ INFN Laboratori Nazionali di Frascati, Frascati, Italy

${ }^{60 a}$ INFN Sezione di Genova, Genova, Italy

${ }^{60 \mathrm{~b}}$ Università di Genova, Genova, Italy

${ }^{61 \mathrm{a}}$ INFN Sezione di Milano-Bicocca, Milano, Italy

${ }^{61 \mathrm{~b}}$ Università di Milano-Bicocca, Milano, Italy

${ }^{62 \mathrm{a}}$ INFN Sezione di Napoli, Napoli, Italy

${ }^{62 \mathrm{~b}}$ Università di Napoli 'Federico II', Napoli, Italy

${ }^{62 \mathrm{c}}$ Università della Basilicata (Potenza), Napoli, Italy

${ }^{62 \mathrm{~d}}$ Università G. Marconi (Roma), Napoli, Italy

${ }^{63 a}$ INFN Sezione di Padova, Padova, Italy

${ }^{63 \mathrm{~b}}$ Università di Padova, Padova, Italy

${ }^{63 \mathrm{c}}$ Università di Trento (Trento), Padova, Italy 
${ }^{64 \mathrm{a}}$ INFN Sezione di Pavia, Pavia, Italy

${ }^{64 \mathrm{~b}}$ Università di Pavia, Pavia, Italy

${ }^{65 a}$ INFN Sezione di Perugia, Perugia, Italy

${ }^{65 \mathrm{~b}}$ Università di Perugia, Perugia, Italy

${ }^{66 a}$ INFN Sezione di Pisa, Pisa, Italy

${ }^{66 \mathrm{~b}}$ Università di Pisa, Pisa, Italy

${ }^{66 \mathrm{c}}$ Scuola Normale Superiore di Pisa, Pisa, Italy

${ }^{67 \mathrm{a} I N F N}$ Sezione di Roma, Roma, Italy

${ }^{67 \mathrm{~b}}$ Università di Roma, Roma, Italy

${ }^{68 \mathrm{a}}$ INFN Sezione di Torino, Torino, Italy

${ }^{68 \mathrm{~b}}$ Università di Torino, Torino, Italy

${ }^{68 c}$ Università del Piemonte Orientale (Novara), Torino, Italy

${ }^{69}$ INFN Sezione di Trieste, Trieste, Italy

${ }^{69 \mathrm{~b}}$ Università di Trieste, Trieste, Italy

${ }^{70}$ Kangwon National University, Chunchon, Korea

${ }^{71}$ Kyungpook National University, Daegu, Korea

${ }^{72}$ Chonbuk National University, Jeonju, Korea

${ }^{73}$ Chonnam National University, Institute for Universe and Elementary Particles, Kwangju, Korea

${ }^{74}$ Korea University, Seoul, Korea

${ }^{75}$ Seoul National University, Seoul, Korea

${ }^{76}$ University of Seoul, Seoul, Korea

${ }^{77}$ Sungkyunkwan University, Suwon, Korea

${ }^{78}$ Vilnius University, Vilnius, Lithuania

${ }^{79}$ National Centre for Particle Physics, Universiti Malaya, Kuala Lumpur, Malaysia

${ }^{80}$ Centro de Investigacion y de Estudios Avanzados del IPN, Mexico City, Mexico

${ }^{81}$ Universidad Iberoamericana, Mexico City, Mexico

${ }^{82}$ Benemerita Universidad Autonoma de Puebla, Puebla, Mexico

${ }^{83}$ Universidad Autónoma de San Luis Potosí, San Luis Potosí, Mexico

${ }^{84}$ University of Auckland, Auckland, New Zealand

${ }^{85}$ University of Canterbury, Christchurch, New Zealand

${ }^{86}$ National Centre for Physics, Quaid-I-Azam University, Islamabad, Pakistan

${ }^{87}$ National Centre for Nuclear Research, Swierk, Poland

${ }^{88}$ Institute of Experimental Physics, Faculty of Physics, University of Warsaw, Warsaw, Poland

${ }^{89}$ Laboratório de Instrumentação e Física Experimental de Partículas, Lisboa, Portugal

${ }^{90}$ Joint Institute for Nuclear Research, Dubna, Russia

${ }^{91}$ Petersburg Nuclear Physics Institute, Gatchina (St. Petersburg), Russia

${ }^{92}$ Institute for Nuclear Research, Moscow, Russia

${ }^{93}$ Institute for Theoretical and Experimental Physics, Moscow, Russia

${ }^{94}$ P.N. Lebedev Physical Institute, Moscow, Russia

${ }^{95}$ Skobeltsyn Institute of Nuclear Physics, Lomonosov Moscow State University, Moscow, Russia

${ }^{96}$ State Research Center of Russian Federation, Institute for High Energy Physics, Protvino, Russia

${ }^{97}$ University of Belgrade, Faculty of Physics and Vinca Institute of Nuclear Sciences, Belgrade, Serbia

${ }^{98}$ Centro de Investigaciones Energéticas Medioambientales y Tecnológicas (CIEMAT), Madrid, Spain

${ }^{99}$ Universidad Autónoma de Madrid, Madrid, Spain

${ }^{100}$ Universidad de Oviedo, Oviedo, Spain

${ }^{101}$ Instituto de Física de Cantabria (IFCA), CSIC-Universidad de Cantabria, Santander, Spain

${ }^{102}$ CERN, European Organization for Nuclear Research, Geneva, Switzerland

${ }^{103}$ Paul Scherrer Institut, Villigen, Switzerland

${ }^{104}$ Institute for Particle Physics, ETH Zurich, Zurich, Switzerland

${ }^{105}$ Universität Zürich, Zurich, Switzerland

${ }^{106}$ National Central University, Chung-Li, Taiwan

${ }^{107}$ National Taiwan University (NTU), Taipei, Taiwan

${ }^{108}$ Chulalongkorn University, Faculty of Science, Department of Physics, Bangkok, Thailand

${ }^{109}$ Cukurova University, Adana, Turkey

${ }^{110}$ Middle East Technical University, Physics Department, Ankara, Turkey

${ }^{111}$ Bogazici University, Istanbul, Turkey

${ }^{112}$ Istanbul Technical University, Istanbul, Turkey

${ }^{113}$ National Scientific Center, Kharkov Institute of Physics and Technology, Kharkov, Ukraine

${ }^{114}$ University of Bristol, Bristol, United Kingdom

${ }^{115}$ Rutherford Appleton Laboratory, Didcot, United Kingdom 


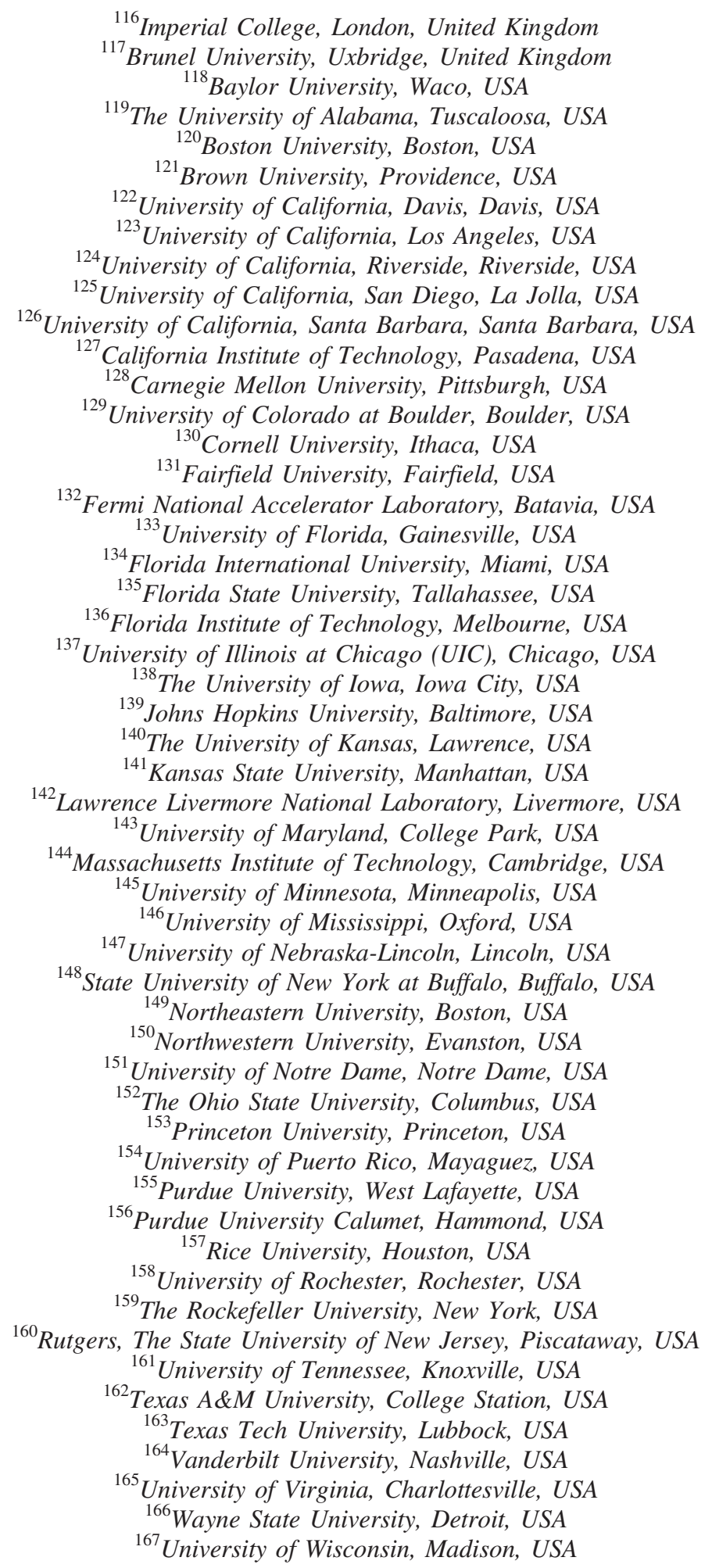

${ }^{\mathrm{a}}$ Deceased.

${ }^{\mathrm{b}}$ Also at Vienna University of Technology, Vienna, Austria.

${ }^{\mathrm{c}}$ Also at CERN, European Organization for Nuclear Research, Geneva, Switzerland.

${ }^{\mathrm{d}}$ Also at Institut Pluridisciplinaire Hubert Curien, Université de Strasbourg, Université de Haute Alsace Mulhouse, CNRS/IN2P3, Strasbourg, France.

${ }^{\mathrm{e}}$ Also at National Institute of Chemical Physics and Biophysics, Tallinn, Estonia.

${ }^{\mathrm{f}}$ Also at Skobeltsyn Institute of Nuclear Physics, Lomonosov Moscow State University, Moscow, Russia. 
${ }^{\mathrm{g}}$ Also at Universidade Estadual de Campinas, Campinas, Brazil.

${ }^{\mathrm{h}}$ Also at Laboratoire Leprince-Ringuet, Ecole Polytechnique, IN2P3-CNRS, Palaiseau, France.

${ }^{\mathrm{i}}$ Also at Joint Institute for Nuclear Research, Dubna, Russia.

${ }^{\mathrm{j}}$ Also at Suez University, Suez, Egypt.

${ }^{\mathrm{k}}$ Also at British University in Egypt, Cairo, Egypt.

${ }^{1}$ Also at Cairo University, Cairo, Egypt.

${ }^{\mathrm{m}}$ Also at Ain Shams University, Cairo, Egypt.

${ }^{\mathrm{n}}$ Also at Sultan Qaboos University, Muscat, Oman.

${ }^{\circ}$ Also at Université de Haute Alsace, Mulhouse, France.

${ }^{\mathrm{p}}$ Also at Ilia State University, Tbilisi, Georgia.

${ }^{\mathrm{q}}$ Also at Brandenburg University of Technology, Cottbus, Germany.

${ }^{\mathrm{r}}$ Also at Institute of Nuclear Research ATOMKI, Debrecen, Hungary.

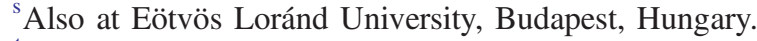

${ }^{\mathrm{t}}$ Also at University of Debrecen, Debrecen, Hungary.

"Also at University of Visva-Bharati, Santiniketan, India.

${ }^{v}$ Also at King Abdulaziz University, Jeddah, Saudi Arabia.

${ }^{\mathrm{w}}$ Also at University of Ruhuna, Matara, Sri Lanka.

${ }^{\mathrm{x}}$ Also at Isfahan University of Technology, Isfahan, Iran.

${ }^{y}$ Also at University of Tehran, Department of Engineering Science, Tehran, Iran.

${ }^{\mathrm{z}}$ Also at Plasma Physics Research Center, Science and Research Branch, Islamic Azad University, Tehran, Iran.

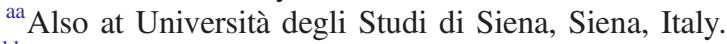

${ }^{\mathrm{bb}}$ Also at Centre National de la Recherche Scientifique (CNRS)_IN2P3, Paris, France.

${ }^{c c}$ Also at Purdue University, West Lafayette, USA.

${ }^{\mathrm{dd}}$ Also at Institute for Nuclear Research, Moscow, Russia.

${ }^{e e}$ Also at St. Petersburg State Polytechnical University, St. Petersburg, Russia.

${ }^{\mathrm{ff}}$ Also at California Institute of Technology, Pasadena, USA.

${ }^{\text {gg }}$ Also at Faculty of Physics, University of Belgrade, Belgrade, Serbia.

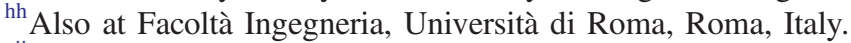

${ }^{\text {ii } A l s o ~ a t ~ S c u o l a ~ N o r m a l e ~ e ~ S e z i o n e ~ d e l l ' I N F N, ~ P i s a, ~ I t a l y . ~}$

${ }^{\mathrm{jj}}$ Also at University of Athens, Athens, Greece.

${ }^{\mathrm{kk}}$ Also at Paul Scherrer Institut, Villigen, Switzerland.

${ }^{11}$ Also at Institute for Theoretical and Experimental Physics, Moscow, Russia.

${ }^{\mathrm{mm}}$ Also at Albert Einstein Center for Fundamental Physics, Bern, Switzerland.

${ }^{n n}$ Also at Gaziosmanpasa University, Tokat, Turkey.

${ }^{\circ o}$ Also at Adiyaman University, Adiyaman, Turkey.

${ }^{\mathrm{pp}}$ Also at Mersin University, Mersin, Turkey.

${ }^{\mathrm{qq}}$ Also at Cag University, Mersin, Turkey.

${ }^{\mathrm{rr}}$ Also at Piri Reis University, Istanbul, Turkey.

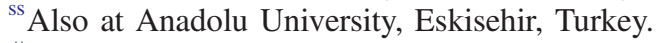

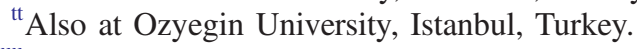

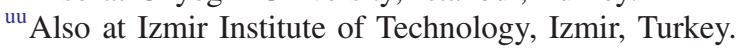

${ }^{\mathrm{vv}}$ Also at Necmettin Erbakan University, Konya, Turkey.

${ }^{\text {ww }}$ Also at Mimar Sinan University, Istanbul, Istanbul, Turkey.

${ }^{\mathrm{xx}}$ Also at Marmara University, Istanbul, Turkey.

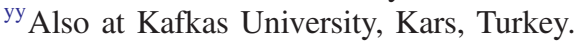

${ }^{\mathrm{zz}}$ Also at Yildiz Technical University, Istanbul, Turkey.

${ }^{\text {aaa }}$ Also at Rutherford Appleton Laboratory, Didcot, United Kingdom.

${ }^{b b b}$ Also at School of Physics and Astronomy, University of Southampton, Southampton, United Kingdom.

${ }^{c c c}$ Also at University of Belgrade, Faculty of Physics and Vinca Institute of Nuclear Sciences, Belgrade, Serbia.

${ }^{\text {ddd }}$ Also at Argonne National Laboratory, Argonne, USA.

${ }^{e e e}$ Also at Erzincan University, Erzincan, Turkey.

${ }^{\mathrm{fff}}$ Also at Texas A\&M University at Qatar, Doha, Qatar.

${ }^{\text {ggg }}$ Also at Kyungpook National University, Daegu, Korea. 